\title{
Disentangling the composite continuum of symbiotic binaries
}

\section{S-type systems}

\author{
A. Skopal \\ Astronomical Institute, Slovak Academy of Sciences, 05960 Tatranská Lomnica, Slovakia \\ e-mail: skopal@ta3.sk
}

Received 2 September 2003 / Accepted 17 May 2005

\begin{abstract}
We describe a method of disentangling the composite, $0.12-5 \mu \mathrm{m}$ continuum of symbiotic binaries. The observed SED is determined by the IUE/HST archival spectra and flux-points corresponding to the optical UBVRI and infrared $J H K L M$ photometric measurements. The modeled SED is given by superposition of fluxes from the cool giant, hot stellar source and nebula including the effect of the Rayleigh scattering process and considering influence of the iron curtain absorptions. We applied this method to $21 \mathrm{~S}$-type symbiotic stars during quiescence, activity and eclipses. We isolated four main components of radiation and determined their properties. (i) Stellar radiation from the giant corresponds to a unique luminosity class normal giants. Characteristic luminosities are $1600 \pm 200$ and $290 \pm 30 L_{\odot}$ for red and yellow giants, respectively in our sample of objects. (ii) Hot object radiation during quiescence consists of the nebular and stellar component. The former radiates at a mean electron temperature of $19000 \mathrm{~K}$ and its amount of emission suggests a mass-loss rate from giants via the wind at $\dot{M}_{\mathrm{W}}=\mathrm{a}$ few $\times 10^{-7} M_{\odot} \mathrm{yr}^{-1}$. Radiation of the latter conforms well with that of a black-body photosphere at a characteristic temperature of $105000 \mathrm{~K}$. The corresponding effective radii are a factor of $\sim 10$ larger than those of white dwarfs, which thus precludes observing the accretor's surface. Extreme cases of AX Per and V443 Her, for which the hot star temperature from the fit is not capable of producing the nebular emission, signal a disk-like structure of the hot stellar source even during quiescence. (iii) Hot object radiation during activity consists of three components - the stellar and the low- and high-temperature nebular radiation. The stellar radiation satisfies that of a black-body photosphere at a low characteristic temperature of $\sim 22000 \mathrm{~K}$ (we call it the 1 st type of outbursts) or at a very high characteristic temperature of $\approx 165000 \mathrm{~K}$ ( 2 nd type of outbursts). All the active objects with a high orbital inclination show features of the 1st-type of outbursts (here Z And, AE Ara, CD $-43^{\circ} 14304, \mathrm{TX} \mathrm{CVn}$, BF Cyg, CH Cyg, CICyg, AR Pav, AX Per), while AG Dra represents the 2nd-type. The presence of a two-temperature type of UV spectrum and an enlargement of effective radii of the stellar source by a factor of $\sim 10$ with respect to the quiescent values during the 1st-type of outburst suggest an expansion of an optically thick medium at the orbital plane in the form of a disk. The low-temperature nebula radiates at a mean electron temperature of $14000 \mathrm{~K}$ and is subject to eclipses, while the high-temperature nebula, which is seen during eclipses as the only component, is characterized by $T_{\mathrm{e}}>30000 \mathrm{~K}$.

Radiative and geometric properties of the main sources of radiation allowed us to reconstruct a basic structure of the hot object during the 1st-type of outburst. There is an edge-on disk around the accretor. Its outer flared rim represents a warm pseudophotosphere of the hot stellar source, whose radiation is Rayleigh attenuated and affected by the iron curtain absorptions in the neutral gas concentrated at the orbital plane. The low-temperature nebula is placed just above/below the disk with a concentration at its edge as to be subject to eclipses and to "see" well the central ionizing source. High above/below the orbital plane, there is a hot nebular emitting region.
\end{abstract}

Key words. methods: data analysis - stars: binaries: symbiotic - stars: fundamental parameters - ultraviolet: stars - accretion, accretion disks

\section{Introduction}

Symbiotic stars are interacting binary systems consisting of a cool giant and a hot compact star. Typical orbital periods are between 1 and 3 years, but can be significantly larger. The mass loss from the giant represents the primary condition for interaction between the binary components. A part of the material lost by the giant is transferred to the more compact companion via accretion from the stellar wind or Roche-lobe overflow. This process generates a very hot $\left(T_{\mathrm{h}} \approx 10^{5} \mathrm{~K}\right)$ and luminous
$\left(L_{\mathrm{h}} \approx 10^{2}-10^{4} L_{\odot}\right)$ source of radiation. How the generated energy is liberated determines two stages of a symbiotic binary. During quiescent phases the hot component releases its energy approximately at a constant rate and spectral distribution. The hot radiation ionizes a fraction of the neutral giant's wind, which gives rise to nebular emission comprising numerous lines of high excitation/ionization and the continuum. As a result the spectrum consists of three components of radiation two stellar and one nebular. For eclipsing systems, the hot star radiation can be attenuated by Rayleigh scattering at the 
position where the mass losing giant is in front (Isliker et al. 1989). During active phases the hot component radiation changes significantly, at least in its spectral distribution, which leads to the 2-3 mag brightening of the object in the optical. A common feature of active phases is a high-velocity mass outflow, which can significantly disrupt the ionization structure of symbiotic nebulae. In some cases the nebular emission disappears entirely at the optical maximum (e.g. BF Cyg), in others dominates the UV/optical spectrum (e.g. AG Dra).

Many particular aspects of this general view have been recently discussed and summarized with outlined problems by Corradi et al. (2003). Current pivotal problems in the symbiotic stars research are connected mainly with understanding the nature of outbursts and the observed accompanied phenomena (Sokoloski et al. 2002; Bode 2003; Skopal 2003c; Lee 2000). In this respect the nature of hot objects in symbiotic stars play a crucial role. A way to determine their fundamental parameters is by modeling the ultraviolet spectrum. A pioneering work in this direction has been made by Kenyon \& Webbink (1984, hereafter $-\mathrm{KW}$ ), who elaborated a grid of synthetic spectra for various binary models. By comparing the calculated and observed ultraviolet continuum colours they suggested the type of the binary model (MS/WD accretor or a single hot star) with the corresponding accretion rate and the hot star temperature. Mürset et al. (1991, hereafter - MNSV) refined their method by developing a more sophisticated model of the nebular continuum accounting for fractional rates of photons capable of ionizing $\mathrm{He}^{+}$and $\mathrm{H}$. This approach requires including a geometrical structure of $\mathrm{He}^{++}$and $\mathrm{H}^{+}$ionized zones in the binary. They fitted the observed IUE spectra scaling the hot star contribution, its temperature (calculated as the Zanstra temperature) and a parameter $X$, which defines the geometry of the nebula in the sense of the STB ionization model (Seaquist et al. 1984, hereafter - STB). Both groups assumed the electron temperature to be constant throughout the nebula, but kept its quantity at $10000 \mathrm{~K}$ and/or $20000 \mathrm{~K}(\mathrm{KW})$ or approximated it by values in a rather narrow interval of $12800-15000 \mathrm{~K}$ for the $\mathrm{H}^{+}$region (MNSV).

The aim of this paper is to introduce a simple method of disentangling the composite SED in the UV/optical/IR continuum of symbiotic binaries (Sect. 3). In our approach we fit observations by a model with 6 basic free parameters - temperatures of the three main emitting sources and scalings of their contributions. In addition we consider effects modifying the continuum - the Rayleigh scattering process and veiling the continuum by a forest of blended Fe II absorptions. In the second part of this paper (Sect. 4) we apply the method to S-type symbiotic binaries during quiescence, activity and eclipses. Particularly we discuss cases in which the modeled parameters lead to a conflict with the observed properties. In Sect. 5 we summarize common characteristics of the isolated components of radiation and their sources.

\section{Observations}

We apply our method (Sect. 3) to well observed S-type symbiotic stars, for which the low-resolution ultraviolet spectroscopy and the broad-band optical and infrared UBVRI and
JHKLM photometry are available. The most important data source we used to cover the ultraviolet domain is the Final archive of the IUE satellite. In the case of AG Peg we analyzed also the spectra taken by the FOS on the board of the HST satellite. We selected spectra with well exposed continuum, taken with the large aperture and the short- and longwavelength prime during one shift. We used the spectra taken during quiescence (if available, at dates of different conjunctions of the binary components), activity and optical eclipses. Main sources of the data covering the infrared region are represented by broad-band multicolour photometric observations published by Munari et al. (1992), Kamath \& Ashok (1999), Taranova (2000), Glass \& Webster (1973) and Feast et al. (1977). Measurements in the $I_{\mathrm{C}}$ and $R_{\mathrm{C}}$ bands of the Cousins system were converted into the Johnson system according to Bessell (1983). In addition, we summarized appropriate parts of light curves (LC) to distinguish stages of quiescence and activity at the time of spectroscopic observations. The main sources of the data here are represented by $U B V R$ photometric observations collected during the last 15 years of our campaign for long-term monitoring of symbiotic stars (Skopal et al. 2004, and references therein). Other data for individual objects are referred in relevant sections. Stellar magnitudes were converted to fluxes according to the calibration of Léna et al. (1999). We dereddened observations with appropriate value of $E_{B-V}$ using the extinction curve of Cardelli et al. (1989).

In Table 1 we list the objects, ephemerides, colour excesses and distances we use in this paper.

\section{Disentangling the composite spectrum}

\subsection{Model of the continuum}

According to the three-component model of radiation (Sect. 1) we can express the observed continuum flux, $F(\lambda)$, of symbiotic stars as a superposition of two stellar components, $F_{\mathrm{h}}(\lambda)$ and $F_{\mathrm{g}}(\lambda)$, from the hot and cool giant star, respectively, and the nebular contribution, $F_{\mathrm{N}}(\lambda)$, as

$F(\lambda)=F_{\mathrm{g}}(\lambda)+F_{\mathrm{h}}(\lambda)+F_{\mathrm{N}}(\lambda)$

in units of erg cm $\mathrm{cm}^{-2} \mathrm{~s}^{-1} \AA^{-1}$. We approximate radiation from the giant by an appropriate synthetic spectrum, $F_{\lambda}^{\text {synth. }}\left(T_{\text {eff }}\right)$, scaled to flux points given by the broad-band photometry. Thus we can write

$F_{\mathrm{g}}(\lambda)=F_{\lambda}^{\text {synth. }}\left(T_{\text {eff }}\right)$.

The effective temperature of the giant, $T_{\text {eff }}$, and the scaling factor of its spectrum determine the bolometric flux at the Earth's surface as

$F_{\mathrm{g}}^{\mathrm{obs}}=\int_{\lambda} F_{\lambda}^{\text {synth. }}\left(T_{\text {eff }}\right) \mathrm{d} \lambda=k_{\mathrm{g}} \int_{\lambda} \pi B_{\lambda}\left(T_{\text {eff }}\right) \mathrm{d} \lambda=k_{\mathrm{g}} \sigma T_{\text {eff }}^{4}$

in units of erg cm $\mathrm{cm}^{-2} \mathrm{~s}^{-1}$. In terms of the giant's luminosity $L_{\mathrm{g}}=$ $4 \pi R_{\mathrm{g}}^{2} \sigma T_{\mathrm{eff}}^{4}$, the scaling factor, $k_{\mathrm{g}}$, can be expressed as

$k_{\mathrm{g}}=\frac{L_{\mathrm{g}}}{4 \pi d^{2} \sigma T_{\mathrm{eff}}^{4}}=\left(\frac{R_{\mathrm{g}}}{d}\right)^{2} \equiv \theta_{\mathrm{g}}^{2}$, 
Table 1. Ephemerides, reddening and distances.

\begin{tabular}{clclll}
\hline \hline Object & $\begin{array}{l}\text { Sp. conj. }{ }^{a} \\
{[\mathrm{MJD}]}\end{array}$ & $\begin{array}{c}P_{\text {orb }} \\
{[\mathrm{day}]}\end{array}$ & $\begin{array}{l}E_{B-V} \\
{[\mathrm{mag}]}\end{array}$ & $\begin{array}{l}d \\
{[\mathrm{kpc}]}\end{array}$ & Ref. \\
\hline EG And & 50683.2 & 482.57 & 0.05 & $0.59^{b}$ & 1,2 \\
Z And & 14625.2 & 757.5 & 0.30 & 1.5 & 3,4 \\
AE Ara & 50217 & 812 & $0.25^{b}$ & $3.5^{b}$ & 5 \\
CD-43 $^{c}$ & 45567 & 1448 & 0.2 & $2.1^{b}$ & 6 \\
T CrB & 47861.7 & 227.57 & 0.15 & 0.96 & $1,7,8$ \\
TX CVn & 45130.5 & 198 & 0.0 & $1.0^{b}$ & 9 \\
BF Cyg & 45395.1 & 757.2 & 0.35 & $3.8^{b}$ & 10 \\
CH Cyg & 45888 & 756 & 0.0 & 0.27 & 11,12 \\
CICyg & 11902 & 855.25 & 0.35 & $2.0^{b}$ & 13,14 \\
V1329 Cyg & 27687 & $958.0^{d}$ & 0.37 & $4.2^{b}$ & 15 \\
LT Del & 45910 & 478.5 & $0.20^{b}$ & $3.9^{b}$ & 31 \\
AG Dra & 43629.2 & 549.73 & 0.08 & $1.1^{b}$ & 16,17 \\
CQ Dra & 42932 & 1703 & 0.10 & 0.178 & 18,19 \\
V443 Her & $43660^{e}$ & 594 & $0.25^{b}$ & $2.2^{b}$ & 20 \\
YY Her & 40637 & 592.8 & 0.20 & $6.3^{b}$ & 3,21 \\
RW Hya & 49512 & 370.4 & 0.10 & $0.82^{b}$ & 22,2 \\
SY Mus & 50176 & 625.0 & $0.35^{b}$ & $1.0^{b}$ & 23 \\
AR Pav & $11266.1^{f}$ & 604.45 & 0.26 & 4.9 & $24-6$ \\
AG Peg & 27664.2 & 812.6 & 0.10 & 0.80 & 3,27 \\
AX Per & 36673.3 & 679.9 & 0.27 & 1.73 & $28-30$ \\
IV Vir ${ }^{g}$ & 49016.9 & 281.6 & $0.20^{b}$ & $1.3^{b}$ & 32 \\
\hline I & & & & &
\end{tabular}

${ }^{a}$ Inferior conjunction of the cool component.

${ }^{b}$ This paper.

${ }^{c} \mathrm{CD}-43^{\circ} 14304$.

${ }^{d}$ Pre-outburst ephemeris.

${ }^{e} \mathrm{JD}_{\mathrm{Min}(\mathrm{U})}$.

${ }^{f}$ A mean linear ephemeris of eclipses.

${ }^{g} \mathrm{BD}-21^{\circ} 3873$

References: 1 - Fekel et al. (2000); 2 - MNSV; 3 - Skopal (1998); 4 - Mikolajewska \& Kenyon (1996); 5 - Mikolajewska et al. (2003); 6 - Schmid \& Nussbaumer (1993); 7 - Selvelli et al. (1992); 8 Belczyński \& Mikolajewska (1998); 9 - Kenyon \& Garcia (1989); 10 - Fekel et al. (2001); 11 - Hinkle et al. (1993); 12 - Viotti et al. (1997); 13 - Aller (1954); 14 - Kenyon et al. (1991); 15 - Schild \& Schmid (1997); 16 - Gális et al. (1999); 17 - Birrielet al. (2000); 18 - Reimers et al. (1988); 19 - Perryman et al. (1997); 20 - Kolotilov et al. (1995); 21 - Munari et al. (1997a); 22 - Schild et al. (1996); 23 Dumm et al. (1999); 24 - Skopal et al. (2001b); 25 - KW; 26 - Schild et al. (2001); 27 - Kenyon et al. (1993); 28 - Skopal (1991); 29 Mikolajewska \& Kenyon (1992a); 30 - Skopal (2000); 31 - Archipova et al. (1995); 32 - Smith et al. (1997).

where $\theta_{\mathrm{g}}$ is the angular radius of the giant. We approximate the hot star continuum by a black-body radiation at a temperature $T_{\mathrm{h}}$. In addition, we consider that it can be attenuated by neutral atoms of hydrogen due to the Rayleigh scattering process. Thus, we express the second term on the right side of Eq. (1) as

$F_{\mathrm{h}}(\lambda)=k_{\mathrm{h}} \times \pi B_{\lambda}\left(T_{\mathrm{h}}\right) \mathrm{e}^{-n_{\mathrm{H}} \sigma_{\lambda}^{\mathrm{R}}}$ where $k_{\mathrm{h}}=F_{\mathrm{h}}(\lambda) / \pi B_{\lambda}\left(T_{\mathrm{h}}\right)$ is a dimensionless scaling factor, which can be expressed in the same form as that for the giant's radiation, i.e.

$k_{\mathrm{h}}=\frac{L_{\mathrm{h}}}{4 \pi d^{2} \sigma T_{\mathrm{h}}^{4}}=\left(\frac{R_{\mathrm{h}}^{\mathrm{eff}}}{d}\right)^{2} \equiv \theta_{\mathrm{h}}^{2}$,

where $L_{\mathrm{h}}, T_{\mathrm{h}}, R_{\mathrm{h}}^{\text {eff }}$ and $\theta_{\mathrm{h}}$ are the luminosity, temperature, effective and angular radius of the hot stellar source, respectively. Further in Eq. (5), $n_{\mathrm{H}}\left[\mathrm{cm}^{-2}\right]$ is the column density of $\mathrm{H}$ atoms and $\sigma_{\lambda}^{\mathrm{R}}\left[\mathrm{cm}^{2}\right]$ is the Rayleigh scattering cross-section for atomic hydrogen. According to Nussbaumer et al. (1989) we calculate the scattering cross-sections as

$\sigma_{\lambda}^{\mathrm{R}}=\sigma_{\mathrm{e}}\left[\sum_{k} \frac{f_{1 k}}{\left(\lambda / \lambda_{1 k}\right)^{2}-1}\right]^{2}$,

where $\sigma_{\mathrm{e}}=6.65 \mathrm{E}-25 \mathrm{~cm}^{-2}$ is the Thomson cross-section, $f_{1 k}$ are oscillator strengths of the hydrogen Lyman lines and $\lambda_{1 k}$ are the corresponding wavelengths. An interesting feature (from the astrophysical point of view) of the $\sigma_{\lambda}^{\mathrm{R}}$ function is its rapid decrease from Ly $\alpha$ to $\lambda 1055 \AA$ (see Fig. 2 of Nussbaumer et al. 1989). This effect should be seen as a rapid increase of the far-UV continuum beyond the $\operatorname{Ly} \alpha$ line to its scattering free values at $\lambda 1055 \AA$. For example, the SWP spectra from the IUE archive begin at $\sim 1140 \AA$, which means that the effect could be detected only for rather low values of $n_{\mathrm{H}} \lesssim 10^{23} \mathrm{~cm}^{-2}$ and on the well exposed spectra.

The last term on the right side of Eq. (1) represents flux in the continuum emitted by the symbiotic nebula. For the purpose of this paper we consider nebular contributions from hydrogen and doubly ionized helium generated by processes of recombination ( $f-b$ transitions) and thermal bremsstrahlung ( $f-f$ transitions). We neglect contributions from singly ionized helium, because its emission coefficient is comparable, in both the profile and the quantity, with that of hydrogen, but it is much less abundant in the ionized medium (to about 10\%). This similarity also makes it difficult to identify any signatures of the emission from the singly ionized helium in the observed spectrum. Therefore, and also for simplicity, we express the nebular flux as

$$
\begin{aligned}
F_{\mathrm{N}}(\lambda)= & \frac{1}{4 \pi d^{2}} \int_{V}\left[\varepsilon_{\lambda}\left(\mathrm{H}, T_{\mathrm{e}}\right) N_{\mathrm{H}^{+}}\right. \\
& \left.+\varepsilon_{\lambda}\left(\mathrm{He}^{+}, T_{\mathrm{e}}\right) N_{\mathrm{He}^{++}}\right] N_{\mathrm{e}} \mathrm{d} V
\end{aligned}
$$

where $d$ is the distance to the object and $\varepsilon_{\lambda}\left(\operatorname{erg~cm} \mathrm{cm}^{3} \AA^{-1}\right)$ is the volume emission coefficient per electron and per ion under consideration, which includes all acts of $f-b$ and $f-f$ transitions. $N_{\mathrm{H}^{+}}, N_{\mathrm{He}^{++}}$and $N_{\mathrm{e}}$ are concentrations of the ionized hydrogen (protons), doubly ionized helium and the electrons, respectively, and $T_{\mathrm{e}}$ is the electron temperature. Further we use the following two assumptions for the symbiotic nebula: (i) both the $\mathrm{H}^{+}$and the $\mathrm{He}^{++}$regions occupy the same volume (in contrast to the usual case, for which the volume $V_{\mathrm{H}^{+}}>$ $\left.V_{\mathrm{He}^{++}}\right)$. Then the ions in such nebula are in the pool of electrons with

$N_{\mathrm{e}}=(1+2 \tilde{a}) N_{\mathrm{H}^{+}}$, 
where

$\tilde{a}=N_{\mathrm{He}^{++}} / N_{\mathrm{H}^{+}}$

represents an average abundance of the doubly ionized helium throughout the nebula. (ii) The nebular radiation is characterized by a uniform electron temperature, $T_{\mathrm{e}}$, which is constant within the nebula. The assumption (i) is based on that the spectra include contributions from all the present ions irrespective of their displacement within the nebula. Then the average abundance $\tilde{a}$ represents a lower limit of that in the real $\mathrm{He}^{++}$region. Specially, in the case that $V_{\mathrm{H}^{+}}=V_{\mathrm{He}^{++}}$, also $\tilde{a}=a\left(\mathrm{He}^{++}\right)$. Contrarily, if $V_{\mathrm{H}^{+}} \gg V_{\mathrm{He}^{++}}$then $\tilde{a} \ll a\left(\mathrm{He}^{++}\right)$, which implies that the contribution from the $\mathrm{He}^{++}$region is very small with respect to that from $\mathrm{H}^{+}$region. These limiting cases can be distinguished from observations. For example, a large quantity of $\tilde{a}$ derived from observations (to say around 0.1 , which can be considered as a maximum given by the helium abundance $a(\mathrm{He})$ ) means that the helium is doubly ionized everywhere in the nebula, i.e. $V_{\mathrm{He}^{++}} \approx V_{\mathrm{H}^{+}}$. The assumption (ii) is verified by good fits of the continuum at spectral regions, where the $\mathrm{He}^{++}$and $\mathrm{H}^{+}$nebular continuum dominate the spectrum (see Figs. 2-22). So, with the aid of Eqs. (9), (10) and the assumption of uniform $T_{\mathrm{e}}$ in the nebula, the expression (8) takes the form

$F_{\mathrm{N}}(\lambda)=\frac{E M}{4 \pi d^{2}} \times \hat{\varepsilon}_{\lambda}\left(\mathrm{H}, \mathrm{He}^{+}, T_{\mathrm{e}}, \tilde{a}\right)$,

where the emission measure $E M=\int_{V} N_{\mathrm{H}^{+}}^{2} \mathrm{~d} V$ and the emission coefficient

$$
\begin{aligned}
\hat{\varepsilon}_{\lambda}\left(\mathrm{H}, \mathrm{He}^{+}, T_{\mathrm{e}}, \tilde{a}\right)= & (1+2 \tilde{a}) \varepsilon_{\lambda}\left(\mathrm{H}, T_{\mathrm{e}}\right) \\
& +\tilde{a}(1+2 \tilde{a}) \varepsilon_{\lambda}\left(\mathrm{He}^{+}, T_{\mathrm{e}}\right) .
\end{aligned}
$$

For small values of $\tilde{a}$, to say $\tilde{a}<0.05, \hat{\varepsilon}_{\lambda} \doteq \varepsilon_{\lambda}\left(\mathrm{H}, T_{\mathrm{e}}\right)+$ $\tilde{a} \varepsilon_{\lambda}\left(\mathrm{He}^{+}, T_{\mathrm{e}}\right)$, which can be applied, for example, to symbiotic nebulae during quiescent phases that are characterized by $V_{\mathrm{H}^{+}}>V_{\mathrm{He}^{++}}$. Finally, according to relations (2), (5) and (11), we can express Eq. (1) in the form

$$
\begin{aligned}
F_{\lambda}= & F_{\lambda}^{\text {synth. }}\left(T_{\text {eff }}\right) \\
& +k_{\mathrm{h}} \times \pi B_{\lambda}\left(T_{\mathrm{h}}\right) \mathrm{e}^{-n_{\mathrm{H}} \sigma_{\lambda}^{\mathrm{R}}} \\
& +k_{\mathrm{N}} \times \hat{\varepsilon}_{\lambda}\left(\mathrm{H}, \mathrm{He}^{+}, T_{\mathrm{e}}, \tilde{a}\right),
\end{aligned}
$$

where $k_{\mathrm{N}}$ is the scaling factor for the nebular component of radiation. According to Eq. (11)

$k_{\mathrm{N}}=\frac{E M}{4 \pi d^{2}} \quad\left[\mathrm{~cm}^{-5}\right]$

\subsubsection{Influence of the line blanketing effect}

This effect represents an additional source of the absorbing spectrum due mostly to Fe II transitions. This so-called "iron curtain" was first identified by Shore \& Aufdenberg (1993) as the origin of the anomalies in the emission-line fluxes resulting from the differential absorption by the environment as the path length along the line-of-sight changes. Generally, the intervening absorption originates in a slab of cool gas that veils

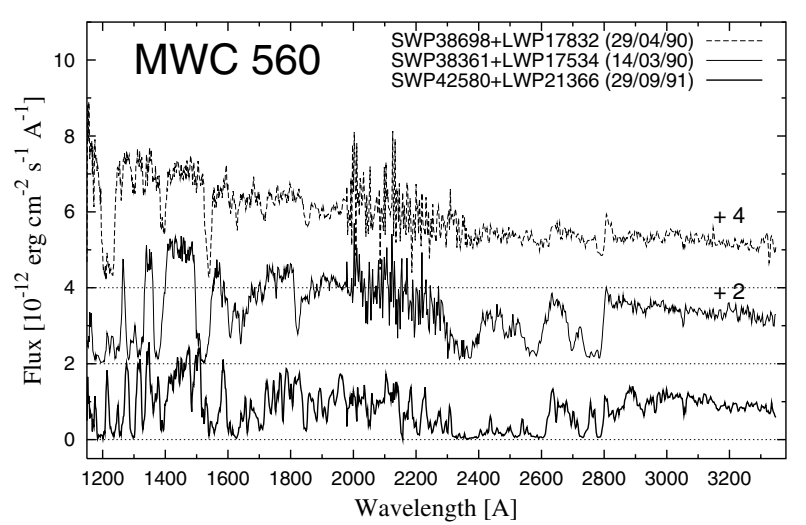

Fig. 1. IUE spectra of MWC 560 obtained on 29/04/90, 14/03/90 and 29/09/91 with different amounts of absorption by the veiling gas.

the source of the hot radiation. In symbiotic binaries the veiling gas can be represented by the neutral wind of the giant, the effect of which is largest at/around the giant's inferior conjunction (e.g. Dumm et al. 1999). In this study we find that such a slab of absorbing gas can temporarily be created at the orbital plane during the $\mathrm{Z}$ And type of outbursts.

The line blanketing effect results in a complex profile of the observed continuum. On the low-resolution spectra broad absorption bands at 1500 to $1800 \AA$ and 2300 to $2800 \AA$ accompanied by spurious emissions are characteristic features. To estimate the level of the continuum at wavelengths with the lowest absorption, we have chosen three representative low-resolution IUE spectra of MWC 560 to obtain a template for a low, modest and strong influence of the continuum by the iron curtain (Fig. 1). With the help of these observations and considering theoretical calculations given by Shore \& Aufdenberg (1993), Horne et al. (1994) and Dumm et al. (1999) we select points of the continuum at $\sim 1280, \sim 1340, \sim 1450, \sim 1500$, ( 1580-1600), around 1800, 1950-2150, ( 2450), 2640 and beyond $2800 \AA$ depending on the amount of absorption.

The resulting effect of the absorbing gas in the spectrum of symbiotic binaries depends also on its relative position with respect to the location of different emitting regions in the system. Generally, radiation from the hot stellar source is absorbed most, while the nebular emission - having its origin in a region located well away from the hot star - is less affected. An additional complication is the superposition of the strong emission lines on the broad absorption bands of the iron curtain. For example, emission of He II 1640, O I 1641] and O III $1664 \AA$ lines fills up in part the underlying absorption bands, which results in an artificial absorption located at/around $1700 \AA$. Its depth reflects the magnitude of the iron curtain effect. This part of the spectrum cannot be used to estimate the level of the continuum.

\subsection{The model}

To determine the giant's continuum we scaled a synthetic spectrum to the broad-band [BVRI]JKLM photometric fluxes (depending on the giant's contribution in the optical). This approach is applicable for S-type symbiotic stars (i.e. with a stellar type of the spectrum; no significant dust emission is 
present in the IR wavelengths). An appropriate synthetic spectrum, which best matches the photometric flux-points, was selected from a grid of models made by Hauschildt et al. (1999). We found it satisfactory to use only a $\left(T_{\text {eff }}, \log (g)\right)$ grid and to smooth the spectrum within $20 \AA$ bins to keep a basic profile of the giant's continuum. The resulting parameters are $T_{\text {eff }}$ and $\theta_{\mathrm{g}}$, which define the observed bolometric flux, $F_{\mathrm{g}}^{\mathrm{obs}}$ (Sect. 3.1). For most of the objects we selected $\log (g) \equiv 0.5$, for $\mathrm{CHCyg}$ and T CrB we put $\log (g) \equiv 0$ and for yellow symbiotics, $1.5-2$, in accordance with their detailed atmospheric analysis (e.g. Smith et al. 1997).

Having determined the radiation from the giant we subtracted its contribution from the selected flux-points of the UV-continuum, $F_{\text {obs }}\left(\lambda_{i}\right)$, and fitted them by the synthetic continuum expressed by Eq. (13), for which the function

$\chi^{2}=\frac{1}{N} \sum_{i=1}^{N}\left[\left(F_{\text {obs }}\left(\lambda_{i}\right)-F_{\mathrm{g}}\left(\lambda_{i}\right)\right)-\left(F\left(\lambda_{i}\right)-F_{\mathrm{g}}\left(\lambda_{i}\right)\right)\right]^{2}$

reaches a minimum. In this way we determined the other 6 free parameters, $k_{\mathrm{h}}, T_{\mathrm{h}}, n_{\mathrm{H}}, k_{\mathrm{N}}, T_{\mathrm{e}}$ and $\tilde{a}$, which define the individual components of radiation. In Eq. (15), $F\left(\lambda_{\mathrm{i}}\right)$ are the theoretical fluxes given by Eq. (13) at selected wavelengths $\lambda_{\mathrm{i}} . N$ is their number. We estimated fluxes $F_{\text {obs }}\left(\lambda_{\mathrm{i}}\right)$ at about 10 to 20 wavelengths between 1250 and $3300 \AA$ depending on quality of the used spectrum. Because of noise, numerous emission lines and absorption features we estimated the continuum fluxes by eye at the wavelengths specified in Sect. 3.1.1. Then, if possible and applicable, we subtracted the contribution from the hot temperature nebula (see Sect. 5.3.1). Second, we prepared a grid of models for reasonable ranges of the fitting parameters and selected that corresponding to a minimum of the $\chi^{2}$ function. We found the initial ranges for $k_{\mathrm{h}}, T_{\mathrm{h}}$ and $n_{\mathrm{H}}$ parameters by comparing the Planck function for different temperatures (15000-40000 K for some active phases) attenuated with $n_{\mathrm{H}} \approx 10^{23} \mathrm{~cm}^{-2}$ (if applicable) to the far-UV spectrum between about 1250 and $1600 \AA$. For the spectra from quiescent phases we adopted in some cases their Zanstra temperature according to MNSV and/or determined their lower limits as introduced in Sect. 4.1. Section 5.2.2 gives more details. The range for $T_{\mathrm{e}}$ can be estimated according to the slope of the near-UV continuum from about $2400 \AA$ to the Balmer jump, where the nebular emission represents a dominant contribution. The average abundance of the doubly ionized helium, $\tilde{a}$, can be estimated from the difference of the continuum level in the range of about $1600 \AA$ to $1980 \AA$ and about $2300 \AA$ to $\sim 3200 \AA$. If we use the IUE spectra, these parts are also well defined. Unfortunately, the part from the beginning of the LWP(R) spectra to about $2300 \AA$ is very noisy and the following region in between $\sim 2300$ and $\sim 2700 \AA$ is often affected by the iron curtain absorptions, which makes it difficult to recognize signatures from the $\mathrm{He}^{++}$emission. Finally, the scaling factor $k_{\mathrm{N}}$ results from the fitting procedure. If the initial grid of models (one model = one combination of the fitting parameters) included the minimum of the $\chi^{2}$ function, then a close grid could be determined around the minimum. Repeating this approach we selected models fitting the observed fluxes within their uncertainties.

\subsection{Uncertainties of the fitting parameters}

To estimate uncertainties of the resulting fitting parameters, we determined a $\chi_{\max }$ quantity, which separates the reliable models from those given by all combinations of the grid parameters. The models with $\chi<\chi_{\max }$ then reconstruct the observed fluxes within their uncertainties. According to evaluation of the well exposed spectra from the IUE archive, they are of about $10 \%$ of the measured values. By preparing many trials we found an approximative relation for $\chi_{\max }$ as

$\chi_{\max } \approx 0.05 \times \tilde{F}_{\text {obs }}$,

where $\tilde{F}_{\text {obs }}$ is the average of the $F_{\text {obs }}\left(\lambda_{\mathrm{i}}\right)$ fluxes. The $\chi_{\max }$ value then bounds the ranges for the fitting parameters in the $\chi\left(n_{\mathrm{H}}, T_{\mathrm{h}}, k_{\mathrm{h}}, \tilde{a}, T_{\mathrm{e}}, k_{\mathrm{N}}\right)$ diagrams. Note that a change of the parameter under consideration within the range of its possible values requires a different combination of the other fitting parameters to keep the $\chi$ value of the corresponding model under the $\chi_{\max }$ limit. The primary source of uncertainties is the accuracy of the measured continuum. The ranges of possible values of the fitting parameters are also given by dependencies of the synthetic continuum on individual parameters. The profile of the far-UV continuum depends strongly on the $n_{\mathrm{H}}$ parameter (Eq. (5)). Any other parameter can exceed its influence on the continuum at these wavelengths. For the cases with a significantly Rayleigh-attenuated far-UV continuum, the $\chi\left(n_{\mathrm{H}}\right)$ function has a rather sharp minimum. A complication here is a lower temperature of the stellar source, $T_{\mathrm{h}} \approx 20000-30000 \mathrm{~K}$ measured during some active phases, because the maximum of the corresponding Planck's function lies around the far-UV region or just beyond it at shorter wavelengths. In this case a higher temperature requires also a slightly higher $n_{\mathrm{H}}$ to keep the fit under $\chi_{\max }$. This dependence mainly enlarges the range of possible values of $T_{\mathrm{h}}$. The profile of the nebular continuum from about $2000 \AA$ to the long-wavelength end of the spectrum depends significantly on the parameters $\tilde{a}$ and $T_{\mathrm{e}}$. For the spectra strongly affected by the iron curtain absorption it is not possible to determine unambiguously the $\tilde{a}$ parameter. In addition, our models (Sects. 4.2-4.22) suggest it to be very small. In conclusion, ranges of the fitting parameters $T_{\mathrm{h}}, n_{\mathrm{H}}, T_{\mathrm{e}}, k_{\mathrm{h}}$ and $k_{\mathrm{N}}$ were estimated to be $20 \%$ to $50 \%$ of their best values.

Uncertainties for the stellar component of radiation from the cool giant are given by those in $T_{\text {eff }}$ and the scaling. The former is proximately $\pm 100 \mathrm{~K}$ (see also Skopal 2000) and the latter is given by that of photometric measurements, which we assumed to be less than $10 \%$. Then the relative mean square error in the observed bolometric flux from the giant is about of $15 \%$.

\section{Application to S-type symbiotic stars}

\subsection{Fitting and derived parameters}

Fitting parameters, $k_{\mathrm{g}}, k_{\mathrm{h}}, k_{\mathrm{N}}$ and corresponding temperatures determine stellar luminosities and emission measure of the nebula (Eqs. (4), (6), (14)). A useful parameter is the ratio of the 
hot star and the cool giant luminosity, which is independent of the distance. According to our notation this can be expressed as

$$
\frac{L_{\mathrm{h}}}{L_{\mathrm{g}}}=\frac{k_{\mathrm{h}}}{k_{\mathrm{g}}}\left(\frac{T_{\mathrm{h}}}{T_{\text {eff }}}\right)^{4} .
$$

At the wavelengths, where the nebular emission dominates the spectrum, one can estimate the upper limit of $E M$ directly from observations by assuming that $F_{\mathrm{N}}(\lambda)=F_{\text {obs }}(\lambda)$, which is usually fulfilled for fluxes in the near-UV region. Then, according to Eq. (11) the emission measure is limited by

$$
E M=4 \pi d^{2} \frac{F_{\mathrm{obs}}(\lambda)}{\hat{\varepsilon}_{\lambda}\left(\mathrm{H}, \mathrm{He}^{+}, T_{\mathrm{e}}, \tilde{a}\right)} .
$$

We calculate the luminosity of the nebula from Eq. (11) as

$L_{\mathrm{N}}=E M \int_{912}^{\infty} \hat{\varepsilon}_{\lambda}\left(\mathrm{H}, \mathrm{He}^{+}, T_{\mathrm{e}}, \tilde{a}\right) \mathrm{d} \lambda$

which is valid for an ionised medium that is optically thick in the Lyman continuum. Finally, we introduce a parameter

$\delta=\frac{E M_{\mathrm{obs}}}{E M_{\mathrm{B}}}$

where $E M_{\mathrm{obs}}$ is the emission measure derived from the model (Eq. (14)) and $E M_{\mathrm{B}}=L_{\mathrm{ph}} / \alpha_{\mathrm{B}}$ results from the equilibrium equation between the rate of ionizing photons, $L_{\mathrm{ph}}$ (photons s${ }^{-1}$ ), and the rate of ionization/recombination acts within the ionized region; $\alpha_{\mathrm{B}}\left(\mathrm{cm}^{3} \mathrm{~s}^{-1}\right)$ stands for the total hydrogen recombination coefficient (STB). Thus $E M_{\mathrm{B}}$ represents a maximum of the nebular emission, which can be produced by the ionizing source under conditions of case $B$ in the hydrogen plasma. This implies that $\delta \leq 1$. Values close to 1 indicate that all ionizing photons are converted by the circumbinary medium and are not lost. Small values of $\delta$ indicate the opposite case. Using the relation for $L_{\mathrm{ph}}$ from Skopal (2001a) the $\delta$ parameter can be expressed as

$\delta=\frac{k_{\mathrm{N}}}{k_{\mathrm{h}}} \alpha_{\mathrm{B}} / f\left(T_{\mathrm{h}}\right)$,

where the function

$$
f\left(T_{\mathrm{h}}\right)=\frac{\pi}{h c} \int_{0}^{912} \lambda B_{\lambda}\left(T_{\mathrm{h}}\right) \mathrm{d} \lambda .
$$

For example, $f\left(8 \times 10^{4} \mathrm{~K}\right)=4.6 \mathrm{E}+25, f\left(1 \times 10^{5} \mathrm{~K}\right)=$ $1.1 \mathrm{E}+26, f\left(1.3 \times 10^{5} \mathrm{~K}\right)=2.7 \mathrm{E}+26$ and $f\left(1.5 \times 10^{5} \mathrm{~K}\right)=$ $4.3 \mathrm{E}+26 \mathrm{~cm}^{-2} \mathrm{~s}^{-1}$. The limiting case, $\delta=1$, determines a lower limit of the temperature of the ionizing source, $T_{\mathrm{h}}^{\mathrm{min}}$, required to produce the observed emission measure. Thus, the number of ionizing photons produced by the hot object radiating at $T_{\mathrm{h}}^{\mathrm{min}}$ just balances the number of recombinations in the continuum. In modeling the ultraviolet continuum by Eq. (13) we often used $T_{\mathrm{h}}=T_{\text {Zanstra }}$ (from MNSV) for a first-model-fit. If the ionizing capacity of the hot stellar source was not consistent with the observed nebular emission $(\delta>1)$ we searched for $T_{\mathrm{h}}^{\mathrm{min}}$. As a temperature change in the range of $T_{\mathrm{h}}>10^{5} \mathrm{~K}$ practically does not influences the resulting fit, we can get $T_{\mathrm{h}}=T_{\mathrm{h}}^{\min }$ by solving equation

$\frac{k_{\mathrm{N}}}{k_{\mathrm{h}}\left(T_{\mathrm{h}}\right)} \alpha_{\mathrm{B}}-f\left(T_{\mathrm{h}}\right)=0$

for parameters $k_{\mathrm{N}}$ and $T_{\mathrm{e}}$ from the first-model-fit.

In the following sections we give results for individual objects with special attention to common properties of their SEDs during quiescent and active phases as well as eclipses. The resulting parameters of the reconstructed continuum are in Tables 2-4. Corresponding synthetic continua together with observations are shown in Figs. 2-22.

\subsection{EG Andromedae}

EG And is a quiet symbiotic star - no active phase has been recorded to date. $U B V$ LCs display a periodic orbitally-related variation of a double-wave in the profile (Fig. 2). Occasionally, $0.3-0.5$ mag flares are observed in the $U$-LC. The infrared photometry was summarized by Skopal (2000). At positions of the inferior conjunction of the giant, the ultraviolet continuum is Rayleigh scattered by H I atoms of the giant's wind, which suggests a high inclination of the orbit (Vogel 1991). To show properties of the ultraviolet continuum we selected IUE observations taken close to the superior conjunction of the giant (SWP42347 + LWP21103, 28/08/91, $\varphi=0.47$ and SWP55897 + LWP31444, 10/09/95, $\varphi=0.52$ ) and at the opposite position (SWP18993 + LWR15045, 13/01/83, $\varphi=0.95$ ).

Radiation from the giant. Fluxes of the infrared photometry can be compared well with the synthetic spectrum calculated for $T_{\text {eff }}=3500 \mathrm{~K}$. The scaling factor, $k_{\mathrm{g}}=2.23 \times 10^{-17}$ yields the integrated flux $F_{\mathrm{g}}^{\mathrm{obs}}=1.9 \times 10^{-7} \mathrm{erg} \mathrm{cm}^{-2} \mathrm{~s}^{-1}$. The timing of the eclipse at $\lambda 1320 \AA$ corresponds to $R_{\mathrm{g}} / A=0.32 \pm$ 0.05 (see Appendix A of Skopal 2001a). For $P_{\text {orb }}=482$ days and the total mass of the binary, $M_{\mathrm{T}} \equiv 2.5 M_{\odot}\left(M_{\mathrm{h}} \equiv 0.6 M_{\odot}\right.$ and elements of Fekel et al. 2000) we get the separation of the stars $A=386 R_{\odot}$, which yields $R_{\mathrm{g}}=124 \pm 19 R_{\odot}$. This quantity can be converted by $\theta_{\mathrm{g}}$ to the distance $d=590 \pm 92 \mathrm{pc}$, which is well within the range of 400 to $1960 \mathrm{pc}$ measured by Hipparcos.

Radiation from the ultraviolet. The last low-resolution spectrum of EGAnd made by IUE (10/09/95) is characterized by a strong nebular emission. Its parameters of our solution $\left(T_{\mathrm{e}}=25000 \pm 3000 \mathrm{~K}, k_{\mathrm{N}}=1.25 \times 10^{15} \mathrm{~cm}^{-5}\right)$ constrain a minimum temperature of the ionizing source, $T_{\mathrm{h}}^{\min }=95000 \mathrm{~K}\left(k_{\mathrm{h}}=1.53 \times 10^{-24}, \delta \sim 1\right.$, Eq. (23) $)$, which makes it just capable of producing the observed emission measure, $E M_{\mathrm{obs}}=5.2 \times 10^{58}(\mathrm{~d} / 590 \mathrm{pc})^{2} \mathrm{~cm}^{-3}$. These parameters imply $L_{\mathrm{h}}=77(d / 590 \mathrm{pc})^{2} L_{\odot}, \theta_{\mathrm{h}}=1.24 \times$ $10^{-12}$ and $R_{\mathrm{h}}=0.032(d / 590 \mathrm{pc}) R_{\odot}$. We adopted this temperature to analyze the remaining two spectra. Observations from 28/08/91 show a significantly smaller amount of nebular emission $\left(k_{\mathrm{N}}=2.5 \times 10^{14} \mathrm{~cm}^{-5}\right)$ radiating at a low temperature of $T_{\mathrm{e}}=13500 \pm 1000 \mathrm{~K}$, even though it was taken at similar orbital phase as the previous spectrum. However, the luminosity of the hot stellar source was comparable: $k_{\mathrm{h}}=1.27 \times 10^{-24}$ $\left(\theta_{\mathrm{h}}=1.1 \times 10^{-12}\right)$, which yields $R_{\mathrm{h}}=0.029(d / 590 \mathrm{pc}) R_{\odot}$ and $L_{\mathrm{h}}=60(d / 590 \mathrm{pc})^{2} L_{\odot}$. The spectrum taken close to the 

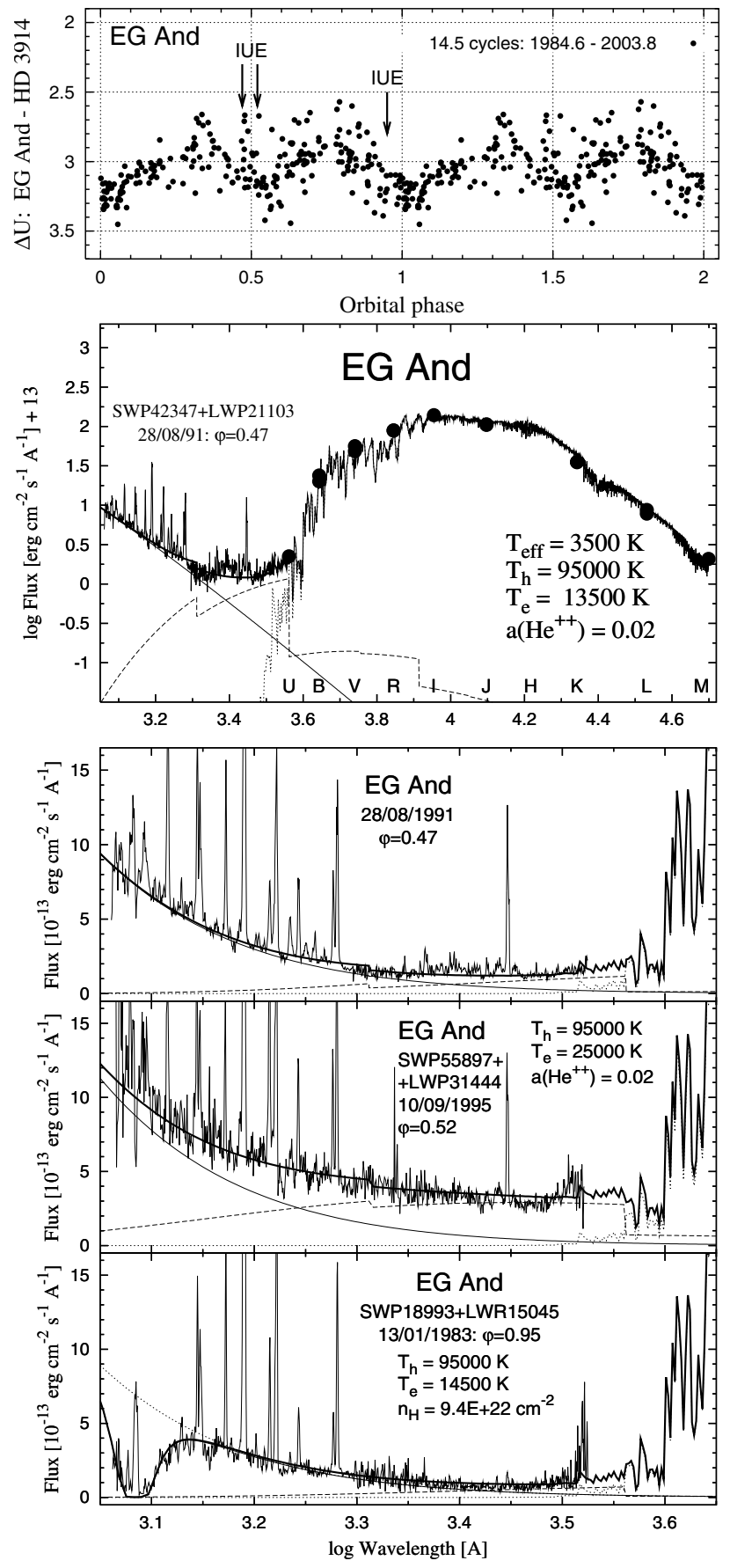

Fig. 2. Reconstructed SED in the continuum of EG And between 0.12 and $5 \mu \mathrm{m}$. The solid thin and dashed lines represent the hot stellar and nebular components of radiation. The solid thick line is the resulting modeled continuum. Top panel shows the phase diagram of $U$ magnitudes. Observations are marked by arrows.

inferior conjunction of the giant (13/01/83) shows a strongly attenuated far-UV continuum by the Rayleigh scattering process $\left(n_{\mathrm{H}}=9.4 \times 10^{22} \mathrm{~cm}^{-2}\right)$ and features of the iron curtain absorptions pronounced at $\sim \lambda 1400 \AA$ and $\sim \lambda 1700 \AA$. However, the stellar radiation can be scaled with approximately the same quantity of $k_{\mathrm{h}}$ as on 28/08/91 and 10/09/95, i.e. if one removes the effect of the Rayleigh scattering, the hot star luminosity remains practically unchanged in all spectra.
The nebular component of radiation is fainter at positions near to the giant's occultation. Skopal (2001a) showed that the variation in the $E M$ follows well that observed in the LCs. Here the amplitude $\Delta m_{E M}=-2.5 \log \left(E M_{\min } / E M_{\max }\right)=0.36$ mag is comparable with that measured photometrically in the $U$ band (Fig. 2). Parameters of the hot star in EG And suggest that the observed hot star luminosity is balanced by accretion processes at a rate of $\dot{M}_{\text {acc }}=$ a few $\times 10^{-8} M_{\odot} \mathrm{yr}^{-1}$ (see Skopal 2005 , for details). Then the observed increase in the nebular emission on 10/09/95 could be caused by a transient increase in the mass-loss rate - an injection of a surplus of emitters into an open H II zone ( $\delta<1$ on 28/08/91) increases the rate of ionization/recombination acts. From this point of view, the 0.3-0.5 mag flares in $U$ result from a variable mass-loss rate from the giant.

\subsection{Z Andromedae}

$\mathrm{Z}$ And is considered as a prototype symbiotic star. The LC is characterized by phases of activity with up to 2-3 mag brightenings, alternating with periods of quiescence (Fig. 3). Recently Skopal (2003a) revealed a high inclination of the $\mathrm{Z}$ And orbit. To show properties of the ultraviolet continuum during quiescence we selected IUE spectra taken at the position when the hot star was in front (SWP18601 + LWR14669, $19 / 11 / 82, \varphi=0.49$; SWP55160 + LWP30952, 19/11/82, $\varphi=$ 0.57 ) and at the opposite position (SWP32845 + LWP12624, $03 / 02 / 88, \varphi=0.99)$. For the active phase we selected spectra taken at the 1985 maximum (SWP27370 + LWP07370, $24 / 12 / 85, \varphi=0.98)$.

Radiation from the giant. We matched the IR fluxes by a synthetic spectrum with $T_{\text {eff }}=3400 \mathrm{~K}$ and scaling $k_{\mathrm{g}}=2.66 \times$ $10^{-18}$, which corresponds to the bolometric flux, $F_{\mathrm{g}}^{\mathrm{obs}}=2.0 \times$ $10^{-8} \mathrm{erg} \mathrm{cm}^{-2} \mathrm{~s}^{-1}$. Our value of $\theta_{\mathrm{g}}$ is in good agreement with that derived from the surface brightness relation for M-giants (Dumm \& Schild 1998), $\theta_{\mathrm{g}}=1.7 \times 10^{-9}$, given by the reddening free magnitudes $K=4.85$ and $J=6.09$ mag. Our value of $\theta_{\mathrm{g}}$ then gives the radius of the giant $R_{\mathrm{g}}=106(d / 1.5 \mathrm{kpc}) R_{\odot}$ and the luminosity $L_{\mathrm{g}} \sim 1400(d / 1.5 \mathrm{kpc})^{2} L_{\odot}$.

Radiation from the ultraviolet: quiescent phase. Features of the iron curtain can be recognized mainly in the shortwavelength part of the spectrum. Our solution for the spectra at $\varphi \sim 0.5$ corresponds to $T_{\mathrm{h}}=120000 \mathrm{~K}=$ $T_{\mathrm{h}}^{\mathrm{min}}$ (see discussion below) and very different electron temperatures, $T_{\mathrm{e}}(19 / 11 / 82)=20500 \pm 2000 \mathrm{~K}$ and $T_{\mathrm{e}}(29 / 06 / 95)=11500 \pm 1000 \mathrm{~K}$. The significant change in $T_{\mathrm{e}}$ by about $10000 \mathrm{~K}$ is observationally given by the change of the slope of the near-UV continuum profile (Fig. 3). The stellar component of radiation was scaled with $k_{\mathrm{h}}=2.73 \times 10^{-24}$, which yields $\theta_{\mathrm{h}}=1.65 \times 10^{-12}, R_{\mathrm{h}}=0.11(d / 1.5 \mathrm{kpc}) R_{\odot}$ and $L_{\mathrm{h}}=2300(d / 1.5 \mathrm{kpc})^{2} L_{\odot}$. The nebular component of the radiation was scaled with $k_{\mathrm{N}}(19 / 11 / 82)=3.6 \times 10^{15} \mathrm{~cm}^{-5}$ and $k_{\mathrm{N}}(29 / 06 / 95)=2.7 \times 10^{15} \mathrm{~cm}^{-5}$. These fitting parameters give $\delta=0.92$ and 1.1 on $19 / 11 / 82$ and 29/06/95, respectively. The case of $\delta \sim 1$ corresponds to a lower limit of the hot star temperature, $T_{\mathrm{h}}^{\min } \sim 120000 \mathrm{~K}$, under which the hot star is not able to produce the observed amount of nebular emission 

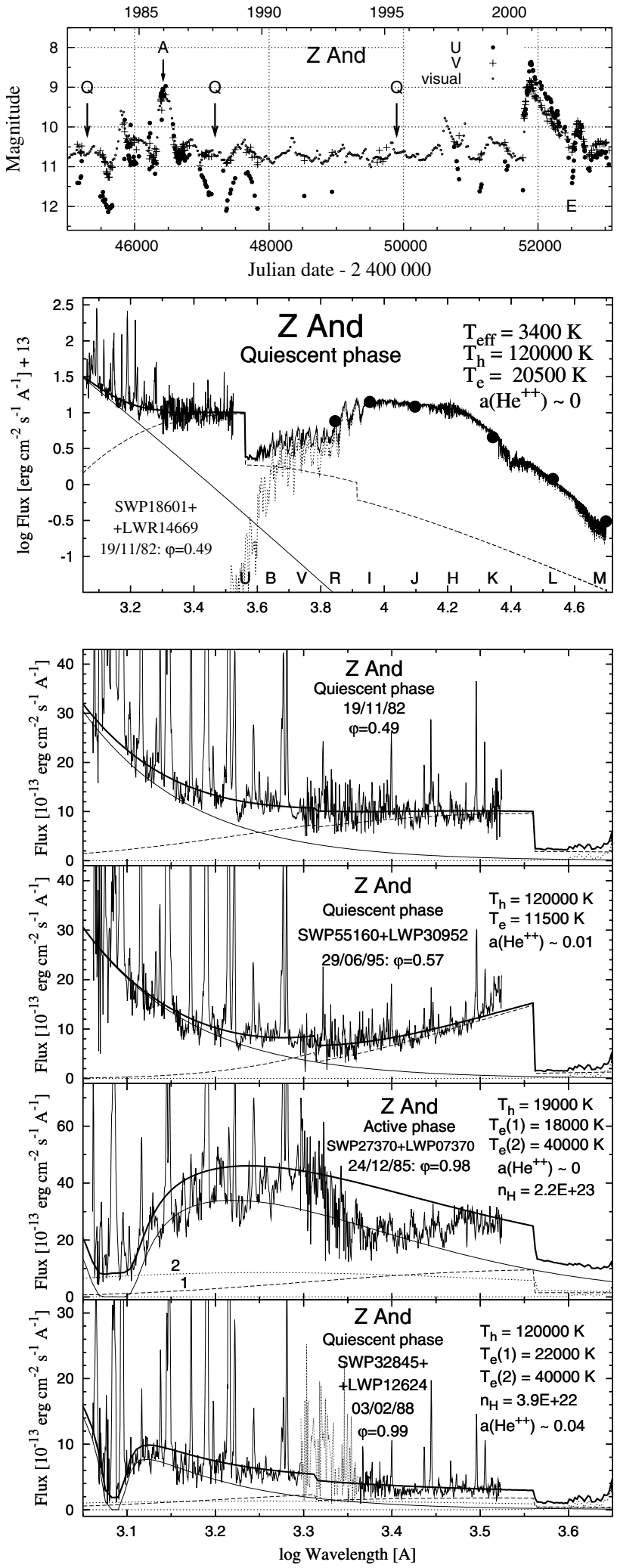

Fig. 3. As in Fig. 2, but for $Z$ And during quiescent and active phases. Dates of IUE observations are marked in the top panel with the LC. Q, $A$ and $E$ denote quiescence, activity and the eclipse effect, respectively.

(Eq. (23)). However, parameters of $Z$ And suggest a very open H II zone (e.g. Fig. 7 of Fernández-Castro et al. 1988), which means that a certain fraction of the ionizing photons escapes the system without being converted to nebular radiation (i.e. $\delta \ll 1)$. This situation requires even higher $T_{\mathrm{h}}$ at the same scaling to balance the measured $E M$. Therefore the real values of $L_{\mathrm{h}}$ and $R_{\mathrm{h}}^{\text {eff }}$ are larger/smaller than we derived from our model for the $T_{\mathrm{h}}^{\mathrm{min}}$ temperature. The spectrum from 03/02/88 $(\varphi \sim 0)$ is characterized by the Rayleigh attenuated far-UV continuum with $n_{\mathrm{H}} \sim 3.9 \times 10^{22} \mathrm{~cm}^{-2}$ and features of the iron curtain that are more pronounced than at the opposite binary position. This can be interpreted in terms of the atmospheric eclipse due to Rayleigh scattering of the far-UV photons on the hydrogen atoms of the neutral giant's wind (Isliker et al. 1989). During quiescent phases this effect is a strong signature of a highly inclined orbit of the binary. A further distinct difference between the spectra taken at $\varphi \sim 0.5$ and $\varphi \sim 0$ is an attenuation of the UV continuum at all wavelengths by a factor of about 2. This effect is present in all IUE spectra of $\mathrm{Z}$ And in the range of phases $\varphi \approx 0 \pm 0.15$. As a result such an extinction process results in lower values of the derived parameters, $\theta_{\mathrm{h}}<1.0 \times 10^{-12}, R_{\mathrm{h}}^{\text {eff }}<0.068(d / 1.5 \mathrm{kpc}) R_{\odot}$ and $L_{\mathrm{h}}>860(d / 1.5 \mathrm{kpc})^{2} L_{\odot}$. Also the amount of the nebular emission decreased by a factor of about 5 and the parameter $\delta=$ 0.2 for $k_{\mathrm{h}}=1.0 \times 10^{-24}$, which reflects a significant attenuation/loss of the nebular photons in the direction of the observer at $\varphi \sim 0$.

Radiation from the ultraviolet: active phase. The selected spectrum is characterized by a strong Rayleigh attenuation of the far-UV continuum and the effect of the iron curtain absorption, which drastically depressed the level of the continuum in between 2150 and $3000 \AA$ (Fig. 3). Note that during activity these effects are also significant out of the occultation by the giant (e.g. on SWP22684 + LWP03099, $\varphi=0.15$ ). The temperature of the stellar component of the radiation significantly decreased to $T_{\mathrm{h}} \sim 19000 \mathrm{~K}$ and is transferred throughout the $\sim 2 \times 10^{23} \mathrm{~cm}^{-2}$ hydrogen atoms on the line of sight. Below we call this stellar component of radiation during active phases the "hot stellar source" (HSS). There are two components of nebular radiation: (i) a low-temperature nebula (LTN) with typical properties of quiescent phases and (ii) a hightemperature nebula (HTN) corresponding to a high electron temperature $\left(T_{\mathrm{e}} \sim 40000 \mathrm{~K}\right)$. The latter is required to fit the farUV continuum. With analogy to AR Pav (see Skopal 2003b), we scaled a $40000 \mathrm{~K}$ hot nebular radiation of hydrogen to the non-zero continuum around the Ly $\alpha$ line. Ignoring the effect of the iron curtain leads to an artificially high $\mathrm{He}^{++}$abundance and too low electron temperature of the LTN (see Fig. 3 of Skopal 2003a).

\subsection{AE Ara}

Visual LC of AE Ara (1987-2004) displays some bright stages alternating with periods of quiescence (Fig. 4). For the purpose of this paper we used the only well exposed low-resolution spectrum from the IUE archive (SWP06793 + LWR05784, $08 / 10 / 79, \varphi=0.53$ ). To flatten its pronounced $2200 \AA$ feature we determined the colour excess, $E_{B-V}=0.25 \pm 0.05$.

Radiation from the giant. A synthetic spectrum with $T_{\text {eff }}=$ $3200 \mathrm{~K}$ matches best the IR fluxes from IJKL photometry 
A. Skopal: Disentangling the composite continuum of symbiotic binaries. I.
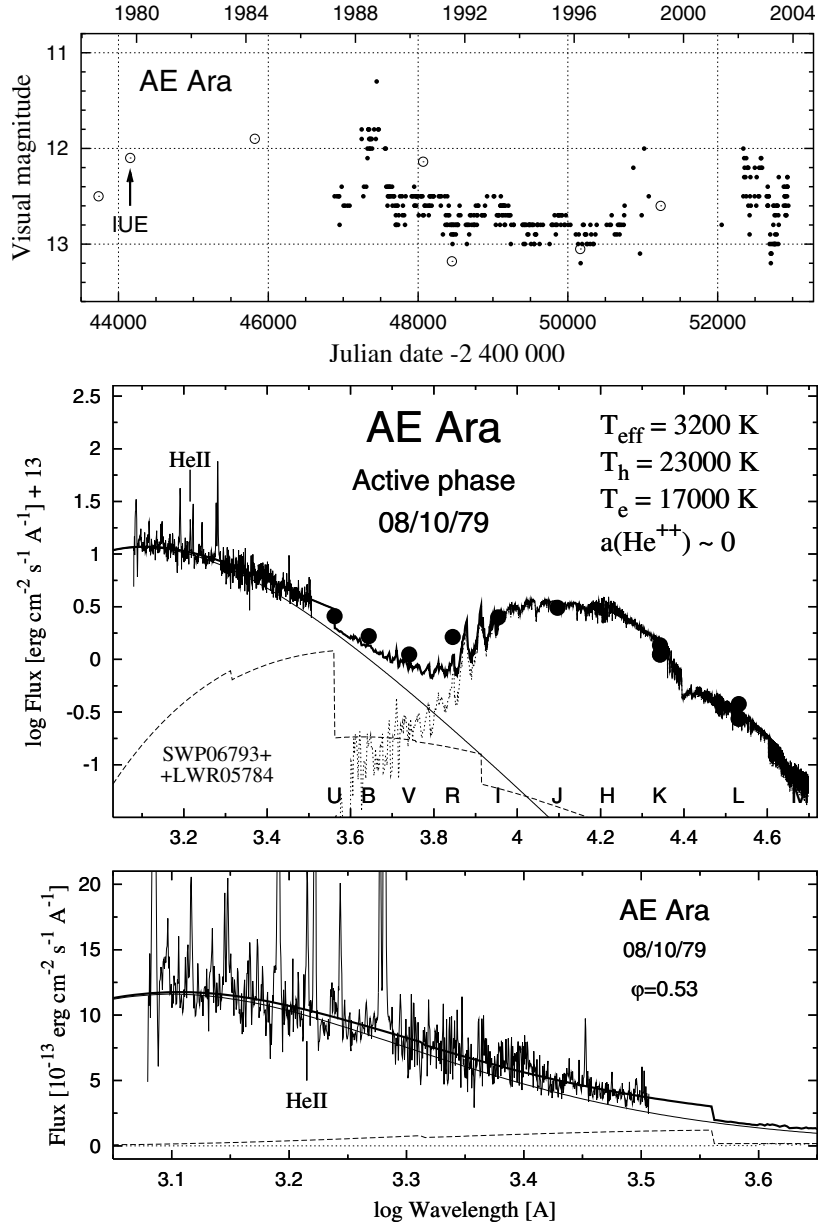

Fig. 4. Top: visual light curve of AR Ara. At the time of IUE observations the system was at a higher level of its activity. Data are from A. Jones (•, private communication) and Mikolajewska et al. (2003) (०). Middle and bottom: reconstructed SED shows typical characteristics of an active phase of the system.

(Allen \& Glass 1974; Munari et al. 1992). Its scaling, $k_{\mathrm{g}}=8.1 \times 10^{-19}$, yields $F_{\mathrm{g}}^{\mathrm{obs}}=4.8 \times 10^{-9} \mathrm{erg} \mathrm{cm}^{-2} \mathrm{~s}^{-1}$. If we adopt the radius of the giant, $R_{\mathrm{g}}=130-150 R_{\odot}$, in agreement with the empirical relations between the spectral type and $T_{\text {eff }}$ (e.g. Belle et al. 1999), then $\theta_{\mathrm{g}}$ is transformed to $d=3.5 \pm$ $0.3 \mathrm{kpc}$, where the uncertainty is given only by the adopted range of the giant's radius. This result is in excellent agreement with that derived from the surface brightness relation for M-giants (Dumm \& Schild 1998). The giant's luminosity $L_{\mathrm{g}}=1800 \pm 300(d / 3.5 \mathrm{kpc})^{2} L_{\odot}$ (Eq. (4)).

Radiation from the ultraviolet. The hot star in AE Ara during the time of the IUE observation was in an active stage with a significant contribution in the optical. A moderate influence of the iron curtain absorptions can be also recognized. Our model identified a warm pseudophotosphere (the HSS) radiating at $T_{\mathrm{h}}=23000+10000 /-3000 \mathrm{~K}$. The scaling factor, $k_{\mathrm{h}}=1.40 \times 10^{-22}$, implies its effective radius, $R_{\mathrm{h}}=1.8(d / 3.5 \mathrm{kpc}) R_{\odot}$ (Eq. (6)). To obtain a better fit of the long-wavelength end of the spectrum, a nebular radiation determined by $T_{\mathrm{e}}=17000 \pm 3000 \mathrm{~K}$ and the scaling, $k_{\mathrm{N}}=3.6 \times$ $10^{14} \mathrm{~cm}^{-5}$, was required. There is no direct signature for the presence of the HTN component in the spectrum. The active
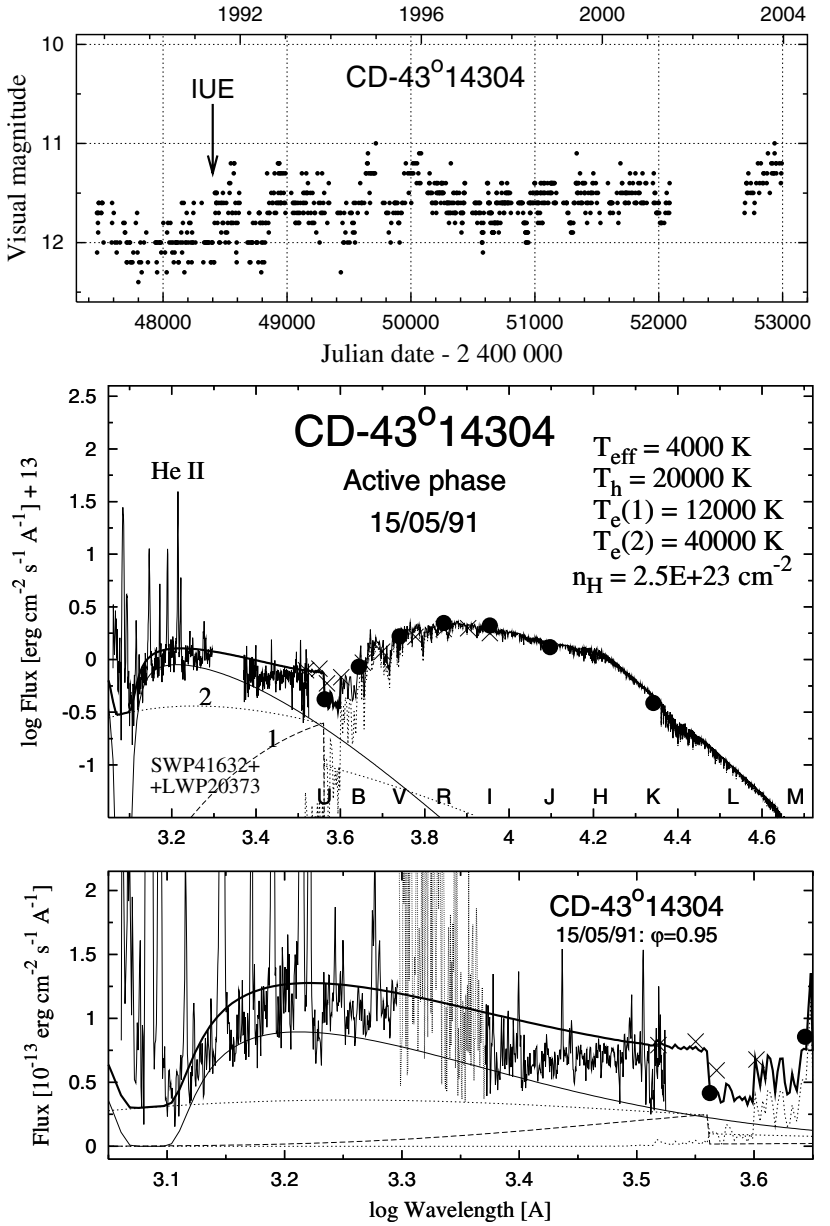

Fig. 5. The SED for CD $43^{\circ} 14304$. It is very similar to that from active phases of other well known systems. Crosses $(\times)$ represent fluxes in the continuum of the optical spectroscopy carried out in July 1991 (Gutiérrez-Moreno et al. 1999). At the time of IUE observations the system just entered an active phase (top panel).

stage of AE Ara is signaled by its two-temperature UV spectrum, which develops during active phases of all the objects with a high orbital inclination (Sect. 5.3.4).

\section{5. $C D-43^{\circ} 14304$}

Visual estimates of this system were regularly carried out by Albert Jones (Fig. 5, private communication). The LC shows periods of a lower and higher level of activity differing by about 1 mag. Only a few multicolour photometric observations were made by Munari et al. (1992) and Gutiérrez-Moreno et al. (1999) in the optical UBVRI and infrared JHK passbands. Schmid \& Nussbaumer (1993) found that the light of $\mathrm{CD}-43^{\circ} 14304$ is reddened with $E_{B-V}<0.2$. The observed index $V-K=4.0$ and that corresponding to the $4000 \mathrm{~K}$ giant, $(V-K)_{\mathrm{g}} \doteq 3.6$ (Belle et al. 1999), yields $E_{B-V}=0.15$. This value represents a lower limit, because of a contribution from other sources in the $V$ band. Therefore we adopted $E_{B-V}=0.2$. There is only one spectrum in the final IUE archive $($ SWP41632 + LWP20373, 15/05/91, $\varphi=0.95)$ applicable to our modeling. The spectrum taken by the long wavelength prime was poorly exposed (Fig. 5). 
Radiation from the giant. The (B)VRIJK photometric measurements can be compared well with a synthetic spectrum of $T_{\text {eff }}=4000 \mathrm{~K}$ scaled with $k_{\mathrm{g}}=1.9 \times 10^{-19}$, which gives $F_{\mathrm{g}}^{\mathrm{obs}}=2.7 \times 10^{-9} \mathrm{erg} \mathrm{cm}^{-2} \mathrm{~s}^{-1}$. If we adopt the giant's radius to be $R_{\mathrm{g}} \equiv 40 R_{\odot}$ as a typical value for the spectral type $\mathrm{K} 7 \mathrm{III}$ (Mürset \& Schmid 1999; Belle et al. 1999) then the angular radius yields $d=2.1 \mathrm{kpc}$. Accordingly, the giant's luminosity $L_{\mathrm{g}}=380(d / 2.1 \mathrm{kpc})^{2} L_{\odot}$.

Radiation from the ultraviolet. The two-temperature type of the ultraviolet spectrum signals an active stage of $\mathrm{CD}-43^{\circ} 14304$. Independently, Gutiérrez-Moreno et al. (1999) found an increase of the intensity shortward of $5000 \AA$ in their optical spectra from 1991 June and July (i.e. taken near to the IUE observations) with respect to the spectrum from 1987 by a factor of 3 at $4000 \AA$. They ascribed this optical brightening to a mild outburst. Our solution indicates a cool shell (i.e. the HSS) re-radiating the hot star photons in the direction of the observer at $T_{\mathrm{h}}=20000+7000 /-3000 \mathrm{~K}$ and scaling of $k_{\mathrm{h}}=2.4 \times 10^{-23}$, which results in a significant contribution in the optical. The spectrum shows the far-UV continuum Rayleigh attenuated by $2.5 \times 10^{23} \mathrm{~cm}^{-2}$ $\mathrm{HI}$ atoms and pronounced features of the iron curtain absorption. The position of the binary at the time of observation $(\varphi=0.95)$ enhances both effects. The effective radius of the shell is $R_{\mathrm{h}}^{\text {eff }}=0.46(d / 2.1 \mathrm{kpc}) R_{\odot}$ and its luminosity $L_{\mathrm{h}}=30(d / 2.1 \mathrm{kpc})^{2} L_{\odot}$ (Eq. (6)). A non-zero level of the Rayleigh attenuated far-UV continuum at/around Ly $\alpha$ indicates a contribution from the HTN with $k_{\mathrm{N}}=1.9 \times$ $10^{14} \mathrm{~cm}^{-5}$ for $T_{\mathrm{e}} \equiv 40000 \mathrm{~K}$. The continuum profile at the end of the LWP spectrum and the following part containing the Balmer jump reflects the presence of a faint radiation from the LTN: $T_{\mathrm{e}} \sim 12000 \mathrm{~K}, k_{\mathrm{N}} \sim 0.5 \times 10^{14} \mathrm{~cm}^{-5}$. Evolution in the $\mathrm{H} \alpha$ line profile along the orbital motion (Schmid et al. 1998 ) is very similar to that observed in eclipsing systems. For example, EG And (Oliversen et al. 1985), V1329 Cyg (Ikeda \& Tamura 2000), AX Per (Iijima 1988), RW Hya (Schild et al. 1996) and SY Mus (Schmutz et al. 1994). This suggests that the orbital plane of $\mathrm{CD}-43^{\circ} 14304$ is highly inclined to the observer, which is consistent with the presence of the twotemperature ultraviolet spectrum.

\subsection{TX Canum Venaticorum}

The historical LC shows the eruptive character of TX CVn. The present active phase is maintained from the last major outburst in 1962 (Fig. 6). Recently, Skopal et al. (2004) revealed two minima in the $U$-LC, whose timing coincides with that of the inferior conjunction of the giant (Kenyon \& Garcia 1989). This suggests a high orbital inclination of TXCVn. Here we used well-exposed IUE spectra taken close to the inferior and superior occultation of the giant (SWP15655 + LWR12083, $05 / 12 / 81, \varphi=0.06$ and SWP05677, 29/06/79, $\varphi=0.57$ ).

Radiation from the giant. A synthetic spectrum of $T_{\text {eff }}=$ $4100 \mathrm{~K}$ matches well the VRJHKL photometric measurements.
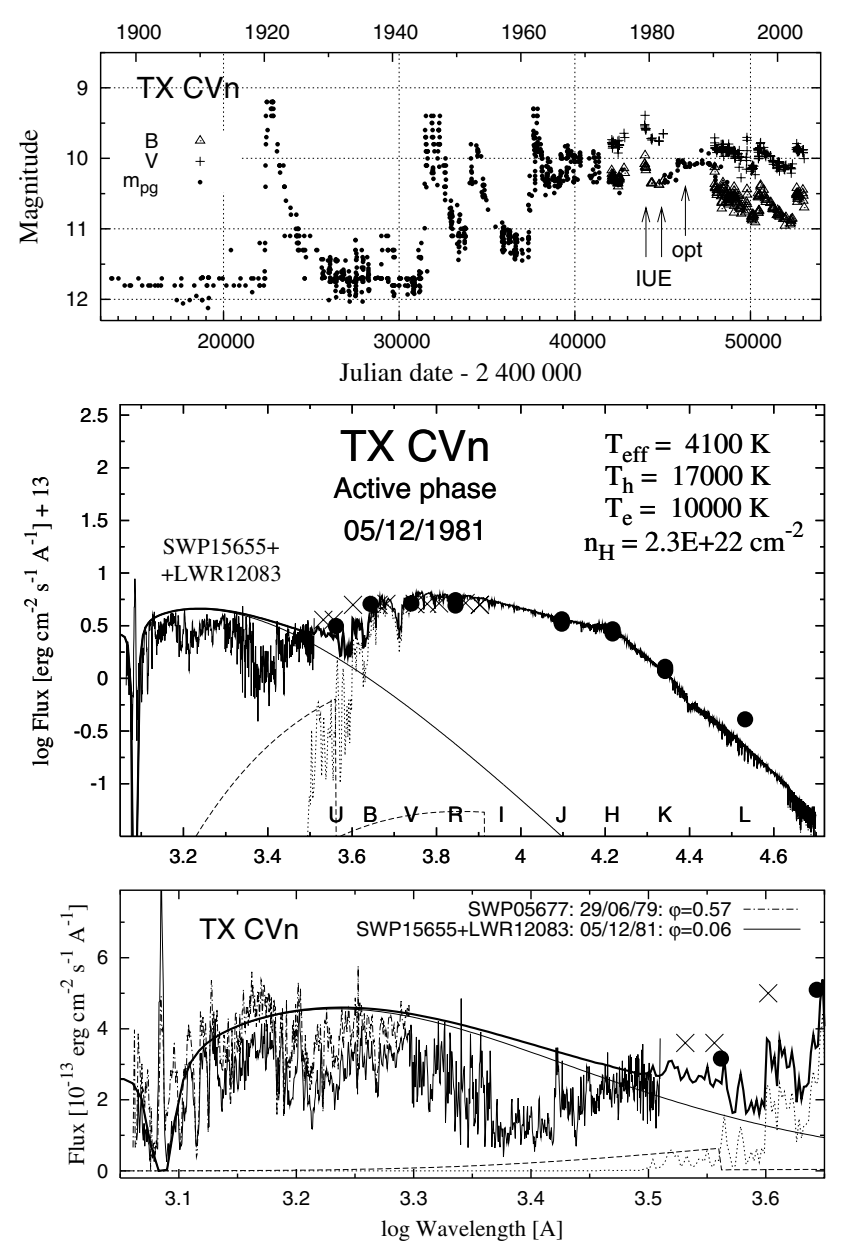

Fig. 6. The observed and modeled SED of TXCVn. The ultraviolet continuum is drastically affected by the iron curtain absorptions and the far-UV part is moderately attenuated by the Rayleigh scattering typical features of active phases of symbiotic stars. Flux-points of the optical spectroscopy $(\times)$ are from Kenyon \& Garcia (1989). Dates of spectroscopic observations are marked by arrows at the top panel.

Its scaling, $k_{\mathrm{g}}=4.5 \times 10^{-19}$, corresponds to $F_{\mathrm{g}}^{\mathrm{obs}}=7.1 \times$ $10^{-9} \mathrm{erg} \mathrm{cm}^{-2} \mathrm{~s}^{-1}$. According to the empirical relations between the spectral type, $V-K$ index and $T_{\text {eff }}$ (e.g. Belle et al. 1999 ), the giant's radius $R_{\mathrm{g}}=30 \pm 10 R_{\odot}$, which then transforms the $\theta_{\mathrm{g}}$ parameter to the distance $d=1.0 \pm 0.3 \mathrm{kpc}$. The corresponding luminosity $L_{\mathrm{g}}=230(d / 1.0 \mathrm{kpc})^{2} L_{\odot}$.

Radiation from the ultraviolet. The profile of the ultraviolet continuum displays characteristics of an active phase in agreement with evolution in the LC (Fig. 6). The effect of Rayleigh scattering and absorptions of the veiling gas are present at very different orbital phases $(\varphi=0.06$ and 0.57$)$, which suggests its distribution around the central star. However it is not possible to determine its geometry more accurately, because the spectrum does not contain emission lines - the shell probably encompasses the whole star core (see also the discussion on the nature of the hot component by Kenyon \& Garcia 1989). Our solution for the 05/12/81 spectrum suggests a black-body radiation at $T_{\mathrm{h}} \sim 17000 \mathrm{~K}\left(k_{\mathrm{h}}=2.6 \times 10^{-22}\right)$ as a dominant component from the hot object. Only a very small nebular contribution is required to fit better the long-wavelength end of the spectrum. However, the line blanketing affects drastically the ultraviolet 

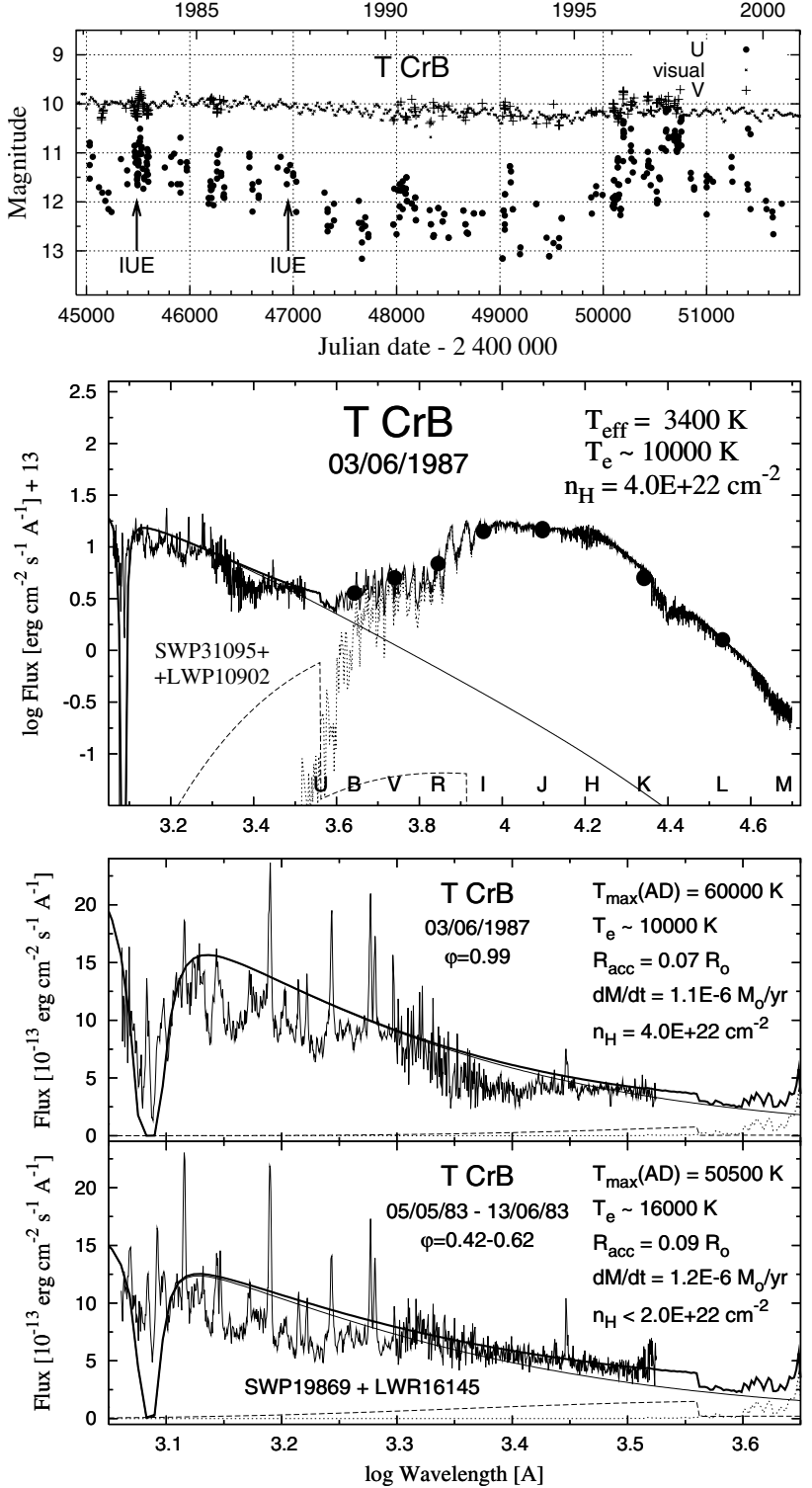

Fig. 7. The UV/optical/IR SED for T CrB. A black-body accretion disk dominates the UV domain with a significant contribution in $U B$ passbands. The Rayleigh scattering and absorptions of the iron curtain affect the UV continuum. The contribution from the nebula is very small. Dates of observations are marked in the LC at the top panel.

continuum, which does not allow us to determine reliably the continuum fluxes and thus to distinguish unambiguously the presence of a faint LTN in the spectrum.

\subsection{TCoronae Borealis}

$\mathrm{T}$ CrB is a symbiotic recurrent nova with two major outbursts, recorded in 1866 and 1946. Recent evolution in the LC shows periods of higher and lower states of activity (e.g. Stanishev et al. 2004, Fig. 7). Currently, it is believed that the hot star luminosity is powered by an accretion process triggered by a mass transfer from the lobe-filling giant. Selvelli et al. (1992) suggested a white dwarf as the accretor to satisfy mainly the ultraviolet IUE observations, while Cannizzo \& Kenyon (1992) tested accretion of a gaseous torus onto a main-sequence star to explain the secondary outburst of the 1946 eruption. To model the ultraviolet continuum we selected observations from the giant's inferior conjunction (SWP31095 + LWP10902, 03/06/87, $\varphi=0.99$ ) and from the opposite site (SWP19869, 01/05/83, $\varphi=0.42$ and LWR16145, 13/06/83, $\varphi=0.62$ - no simultaneous SWP/LWR exposures are available at this position).

Radiation from the giant. The red giant's fluxes can be matched by a synthetic spectrum of $T_{\text {eff }}=3400 \mathrm{~K}$ and the scaling, $k_{\mathrm{g}}=3.13 \times 10^{-18}$, which corresponds to the observed bolometric flux, $F_{\mathrm{g}}^{\mathrm{obs}}=2.37 \times 10^{-8} \mathrm{erg} \mathrm{cm}^{-2} \mathrm{~s}^{-1}$ and the angular radius, $\theta_{\mathrm{g}}=1.77 \times 10^{-9}$. These parameters imply the giant's radius, $R_{\mathrm{g}}=75 \pm 12(d / 960 \mathrm{pc}) R_{\odot}$ and the luminosity, $L_{\mathrm{g}}=680 \pm 230(d / 960 \mathrm{pc})^{2} L_{\odot}$ (Table 1$)$.

Radiation from the ultraviolet. Both spectra display the Rayleigh attenuated far-UV continuum and pronounced features of the iron curtain. This constrains the veiling material surrounding the hot source, being located at/behind the outer rim of the disk, extended vertically from the disk plane, so as to cause the observed absorptions. At $\varphi \sim 0$ the effect of the absorbing slab is enhanced by the cool giant's wind. We estimated flux-points of the continuum with the aid of calculated spectra including the blanketing effect (Shore \& Aufdenberg 1993). We performed modeling of the continuum by substituting the single Planck function in Eq. (13) by a component of radiation from an accretion disk. Then Eq. (13) in the ultraviolet is

$F(\lambda)=k_{\mathrm{AD}} \times F_{\lambda}(\mathrm{AD}) \mathrm{e}^{-n_{\mathrm{H}} \sigma_{\lambda}^{\mathrm{R}}}+k_{\mathrm{N}} \times \hat{\varepsilon}_{\lambda}\left(\mathrm{H}, \mathrm{He}^{+}, T_{\mathrm{e}}, \tilde{a}\right)$.

The function $F_{\lambda}(\mathrm{AD})$ represents a flux distribution from an optically thick accretion disk that radiates locally like a black body. Here we adopted expressions according to the textbook of Warner (1995), recently used also by Skopal (2003b). A fundamental parameter in models with an accretion disk is the disk temperature $T_{\star}=2 T_{\max }$, which determines the slope of the UV continuum. Observations from 03/06/87 suggest $T_{\star} \approx 110000 \mathrm{~K}$ at all wavelengths. We found that it is possible to match the selected points of the continuum by very different sets of model parameters. Below we summarize all possible solutions to select ranges of fitting parameters that are physically plausible. In all calculations we adopted the core mass of the accretor to be $1.37 M_{\odot}$ and the orbital inclination $i=67^{\circ}$ (Stanishev et al. 2004).

1. Solutions with the accretion disk only:

(a) if the radius $R_{\mathrm{h}} \sim R_{\mathrm{WD}}$ (we adopted $R_{\mathrm{h}}=0.01 R_{\odot}$ ) then the temperature required by the slope of the continuum, $T_{\star} \sim 110000 \mathrm{~K}$, corresponds to the mass accretion rate $\dot{M}_{\text {acc }} \sim$ a few $\times 10^{-9} M_{\odot} \mathrm{yr}^{-1}$, which yields $L_{\text {acc }} \sim$ a few $\times L_{\odot}$. These values are much lower than those given by observations, $L_{\mathrm{obs}}(\mathrm{AD})=\int_{\lambda} F_{\lambda}(\mathrm{AD}) \mathrm{d} \lambda / \cos (i) \sim$ $300 L_{\odot}$;

(b) if the radius $R_{\mathrm{h}}>R_{\mathrm{WD}}$ (we tested a range of 0.10 to $0.05 R_{\odot}$ ), the $T_{\star}$ temperature can be satisfied by $\dot{M}_{\text {acc }} \sim$ $10^{-6} M_{\odot} \mathrm{yr}^{-1}$ corresponding to $L_{\mathrm{acc}} \sim L_{\mathrm{obs}}(\mathrm{AD})$.

2. Solutions with the accretion disk and nebula. These models are not unambiguous. Models with a higher $T_{\star}$ express 
better only the far-UV region, which then requires a strong nebular contribution to fit the near-UV part of the spectrum. (a) The case of $R_{\mathrm{h}} \sim R_{\mathrm{WD}}$ and a large nebular contribution leads to $T_{\star} \gg 110000 \mathrm{~K}, L_{\text {acc }} \sim 10^{3} L_{\odot}$ and $\dot{M}_{\text {acc }} \sim 1-10 \times 10^{-7} M_{\odot} \mathrm{yr}^{-1}$. These models are not consistent with observations in too high $L_{\text {acc }}$ and the nebular emission. Models with small contributions from the nebula converge to the cases of point 1 (a) above;

(b) Solutions for $R_{\mathrm{h}}>R_{\mathrm{WD}}$ correspond to $T_{\star} \sim 1.1-1.3 \times$ $10^{5} \mathrm{~K}, \dot{M}_{\mathrm{acc}} \sim 1-1.6 \times 10^{-6} M_{\odot} \mathrm{yr}^{-1}$ and $L_{\mathrm{acc}} \sim$ $L_{\text {obs }}(\mathrm{AD}) \sim 300-400 L_{\odot}$. The emission measure is small and it is not possible to determine $T_{\mathrm{e}}$ unambiguously.

Our modeling of the SED of T CrB shows that a major fraction of the UV radiation is produced by an optically thick accretion disk. Models whose parameters are consistent with observations result from an accretion process at high accretion rates of $\dot{M}_{\text {acc }} \sim 1-1.6 \times 10^{-6} M_{\odot} \mathrm{yr}^{-1}$ onto the accretor with $R_{\mathrm{h}}>R_{\mathrm{WD}}$ (Fig. 7). These results, however, do not support the presence of the white dwarf as the accretor in $\mathrm{T} \mathrm{CrB}$.

\subsection{BFCygni}

BF Cyg is an eclipsing symbiotic star (Skopal 1992, Fig. 8 here). Its historical LC shows three basic types of active phases - nova-like and Z And type of outbursts and short-term flares (Fig. 1 of Skopal et al. 1997). Here we selected observations from the optical maximum (SWP39163 + LWP18251, $30 / 06 / 90, \varphi=0.59)$, the eclipse effect (SWP41531+ LWP20275, 01/05/91, $\varphi=0.0$ ), a transition to quiescence (SWP44370 + LWP22778, 10/04/92, $\varphi=0.46)$ and a quiescence (SWP58385 + LWP32695, 29/09/96, $\varphi=0.61$ ).

Radiation from the giant. Fluxes of the photometric measurements can be compared with a synthetic spectrum of $T_{\text {eff }}=$ $3400 \mathrm{~K}$ (Fig. 8). Its scaling, $k_{\mathrm{g}}=4.5 \times 10^{-19}$, corresponds to the bolometric flux, $F_{\mathrm{g}}^{\mathrm{obs}}=5.9 \times 10^{-9} \mathrm{erg} \mathrm{cm}^{-2} \mathrm{~s}^{-1}$. Empirical relations between the spectral type and $T_{\text {eff }}$ suggest the giant's radius $R_{\mathrm{g}} \sim 150 R_{\odot}$, which yields the distance $d \sim 3.8 \mathrm{kpc}$. On the other hand the contact times of the 1991 eclipse give $R_{\mathrm{g}} / A=0.54 \pm 0.02$, which suggests a very large radius of the giant (Skopal et al. 1997). A solution for the spectroscopic orbit (Fekel et al. 2001) yields the Roche lobe radius $R_{\mathrm{L}} / A=0.49$. These results imply the lobe-filling giant in BF Cyg. However, the broad eclipse profile may be partly caused by attenuation of the optical light in the extended dense atmosphere of the giant. Note that the SWP42356 spectrum from 29/08/91 shows a steep far-UV profile, which signals the out-of-eclipse position, while the brightness in the optical remains at the level of the total eclipse. Therefore here we adopted $R_{\mathrm{g}}=150 R_{\odot}$.

Active phase. The continuum is drastically depressed by the iron curtain absorptions and the far-UV continuum profile indicates a hydrogen column density with $n_{\mathrm{H}} \sim 5 \times 10^{22} \mathrm{~cm}^{-2}$ and a contribution from the HTN. Other spectra from the maximum are of the same type, i.e. its profile does not depend on the orbital phase. Only very faint emissions of Si IV 1403, He II 1640 and O III $1664 \AA$ can be still recognized. Our solution suggests the presence of a strong stellar component $\left(T_{\mathrm{h}} \sim 21500 \mathrm{~K}, k_{\mathrm{h}} \sim\right.$ $\left.1.8 \times 10^{-21}\right)$ superposed on the HTN emission $\left(T_{\mathrm{e}} \sim 42000 \mathrm{~K}\right.$,
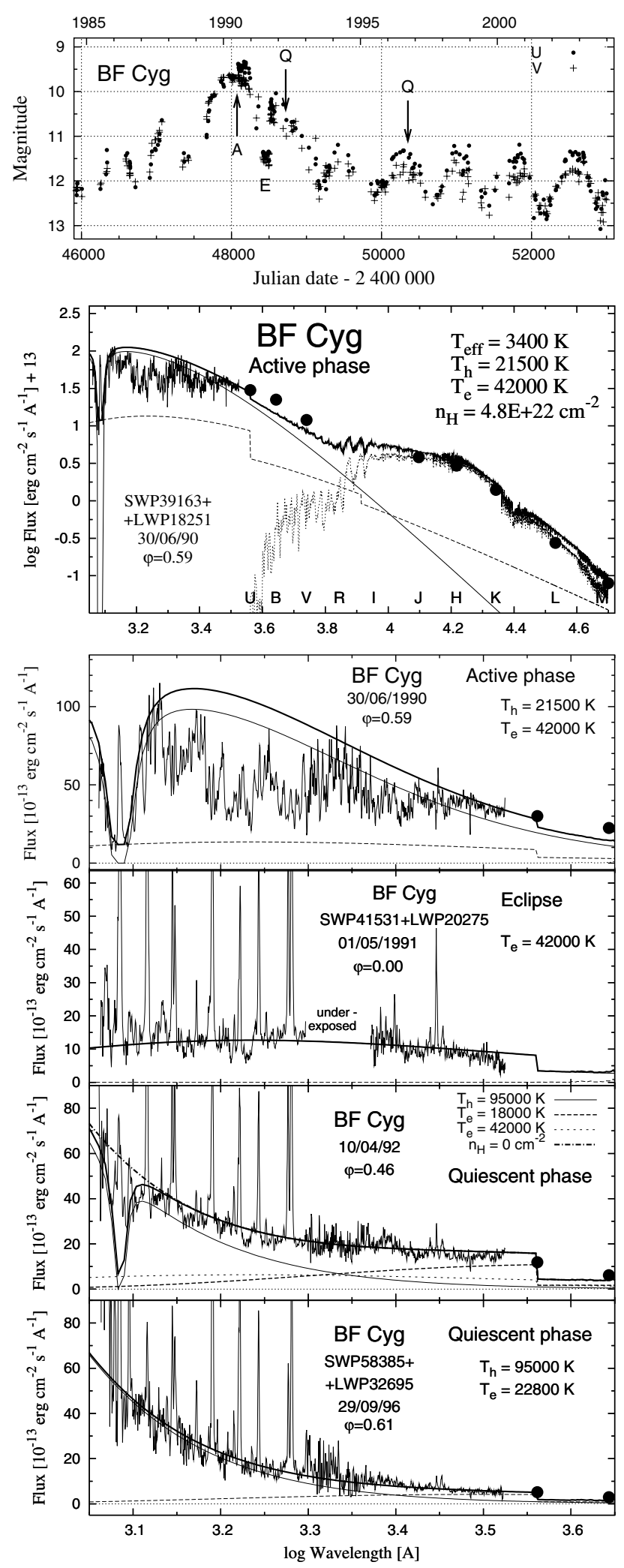

Fig. 8. The SEDs of BF Cyg during the maximum of the star's brightness (denoted by $A$ at the top panel), eclipse (E) and quiescence ( $Q$ ). Dates of observations are marked in the LC at the top panel.

$k_{\mathrm{N}} \sim 6.9 \times 10^{15} \mathrm{~cm}^{-5}-$ as derived from the following eclipse). Both components dominate the optical and in part influence also the IR wavelengths. This composition explains colour 
indices during the totality of the 1991-eclipse, which also reflect a combined spectrum (Skopal 1992).

Eclipse. The ultraviolet continuum from the 1991-eclipse is flat in profile radiating at a level of $\sim 10^{-12} \mathrm{erg} \mathrm{cm}^{-2} \mathrm{~s}^{-1} \AA^{-1}$. We ascribe this component to the emission from the HTN $\left(T_{\mathrm{e}} \sim 42000 \mathrm{~K}, E M_{\mathrm{HTN}} \sim 1.1 \times 10^{61}(d / 3.8 \mathrm{kpc})^{2} \mathrm{~cm}^{-3}\right.$ and $\left.L_{\mathrm{HTN}} \sim 2100(d / 3.8 \mathrm{kpc})^{2} L_{\odot}\right)$.

Transition to quiescence. First we subtracted the HTN contribution and fitted the rest fluxes adopting the hot star temperature $T_{\mathrm{h}}=95000 \mathrm{~K}=T_{\mathrm{h}}^{\min }(\delta=1$, Eq. (23)). The presence of the HTN emission is given by observations from the preceding eclipse. A high $T_{\mathrm{h}}$ here is required by a large emission measure $E M=1.2 \times 10^{61}(d / 3.8 \mathrm{kpc})^{2} \mathrm{~cm}^{-3}$ in total (Table 3$)$. We note that a fraction of the nebular emission can be produced by collisions as suggested by the presence of the HTN (Sect. 5.3.1). This would allow the ionizing source to be cooler than $T_{\mathrm{h}}^{\mathrm{min}}$. Iron curtain features are relatively faint and the far-UV spectrum is attenuated with only $n_{\mathrm{H}} \leq 10^{22} \mathrm{~cm}^{-2}$. If one ignores influence of the iron curtain a formal good fit with $T_{\mathrm{h}}=60000 \mathrm{~K}$ is also possible (Fernández-Castro et al. 1990).

Quiescent phase. The stellar component has practically the same properties as that from the transition period, but without signatures of more pronounced absorptions from the veiling neutral gas. However, the nebular component $\left(T_{\mathrm{e}}=\right.$ $22800 \mathrm{~K}$ ) decreased by a factor of about 4 , to $E M=3.1 \times$ $10^{60}(d / 3.8 \mathrm{kpc})^{2} \mathrm{~cm}^{-3}$, despite of that both the spectra were taken at the same orbital phase $(\varphi \approx 0.5)$. A decrease of the emission measure at the same ionizing capacity of the hot star implies that a fraction of the ionizing photons escapes the system without being converted into the nebular emission, i.e. the parameter $\delta$ decreased and the nebula became more open than during the transition period. A constant mass-loss rate from the giant would mean that the hot star wind, which developed during the activity, caused a surplus of ionizations. In part it is itself a subject of ionization and in part it creates a fraction of the HTN by collisions. Otherwise the mass-loss rate from the giant had to significantly decreased.

\subsection{CHCygni}

$\mathrm{CHCyg}$ is the brightest symbiotic star in the infrared passbands $(J \sim 0.9, K \sim-0.6 \mathrm{mag})$. It is at $270 \pm 66 \mathrm{pc}$ (Viotti et al. 1997). We consider $\mathrm{CHCyg}$ to be a triplestar system consisting of the inner, 756-day period symbiotic binary, which is moving in a common 5300-day period outer orbit with another red giant (Hinkle et al. 1993; Skopal et al. 1996). To demonstrate evolution in the SED during active phases of $\mathrm{CHCyg}$ we selected observations from its 1992-95 activity: before the 1994 eclipse in the inner binary (SWP47771 + LWP25627, 30/05/93, $\varphi=0.30$ ), during the totality (SWP52989 + LWP29646, 05/12/94, $\varphi=0.03$ ) and after the eclipse (SWP54254 + LWP30341, 30/03/95, $\varphi=0.18$ ).

Radiation from the giant. The slope of the VRIJ fluxes suggests a very low temperature of the giant(s) in the system. We matched the $U B V$ fluxes from the giant and the observed fluxes in the RIJKLM bands by a synthetic spectrum of $T_{\mathrm{eff}}=$ $2600 \mathrm{~K}$ (see Appendix B of Skopal et al. 2002a, for details).
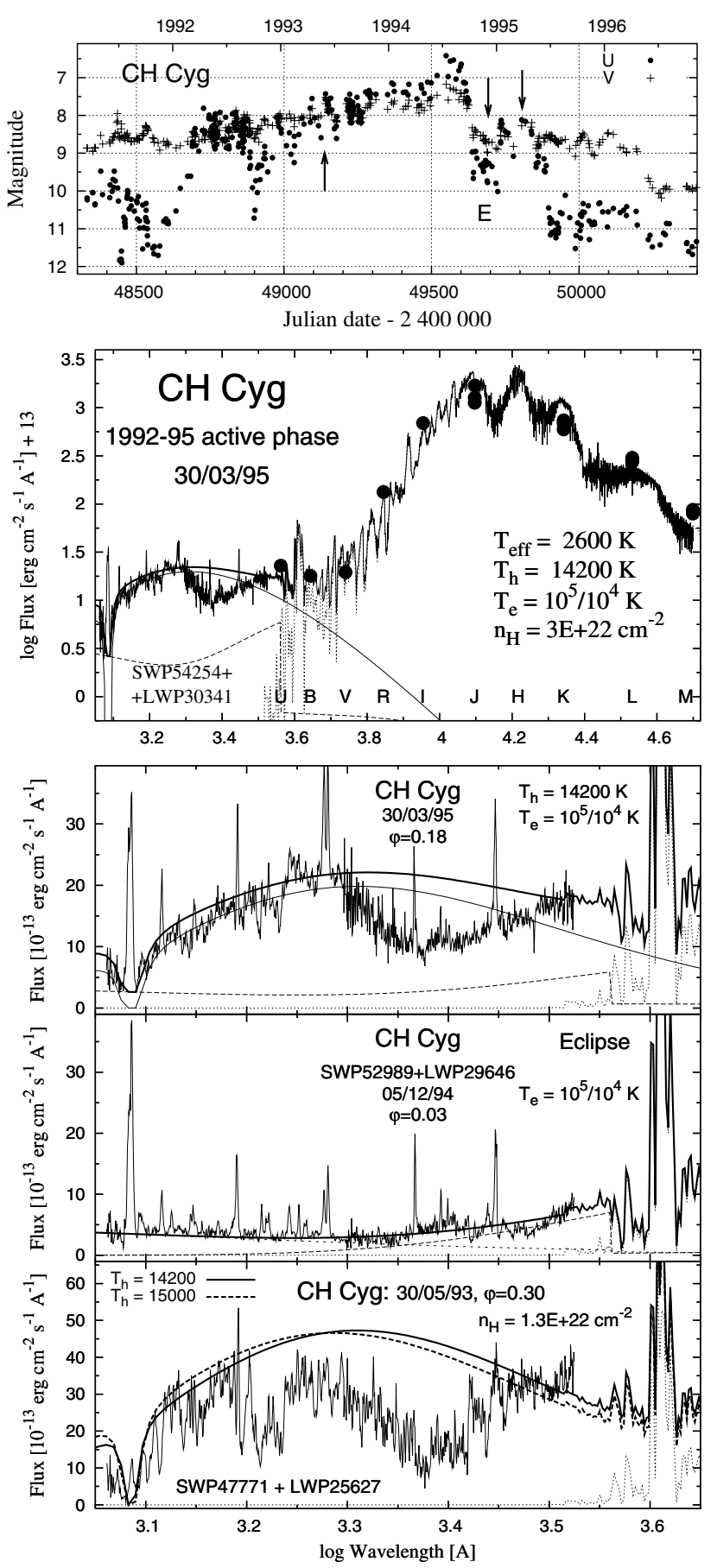

Fig. 9. Example of the SED for CHCyg during its 1992-95 active phase. Spectra prior to, in and after the 1994 eclipse were selected. Corresponding dates are marked at the LC on the top panel.

Corresponding parameters, $F_{\mathrm{g}}^{\mathrm{obs}}=2.4 \times 10^{-6} \mathrm{erg} \mathrm{cm}^{-2} \mathrm{~s}^{-1}$ and $\theta_{\mathrm{g}}=3.1 \times 10^{-8}$ yield the giant's radius $R_{\mathrm{g}} \sim 370(d / 270 \mathrm{pc}) R_{\odot}$ and the luminosity $L_{\mathrm{g}} \sim 5600(d / 270 \mathrm{pc})^{2} L_{\odot}$. These parameters probably belong in major part to the giant at the outer orbit in the triple-star model. We note that the presence of the two red giants in the system has been suggested by the timing of photometric eclipses (e.g. Skopal 1997) and by the COAST interferometric measurements at $0.9 \mu \mathrm{m}$ (Young et al. 2000). 
Eclipse. The spectrum taken during the totality is almost flat in profile with a moderate increase of fluxes from about $2000 \AA$ to the long-wavelength end of the spectrum (Fig. 9). On the radio maps, Crocker et al. (2001) found two components of nebular emission. One is of thermal nature located around the central bright peak and another one is of non-thermal nature associated with the extended regions. Eyres et al. (2002) suggested that the extended emission has electron temperatures of $\sim 100000 \mathrm{~K}$. Therefore we compared a $10^{5} \mathrm{~K}$ hot nebular radiation to the SWP spectrum and filled in the rest of the IUE spectrum by a $10^{4} \mathrm{~K}$ nebular radiation. The resulting fit expresses satisfactorily the overall profile. The longwavelength part of the spectrum is affected by the iron curtain absorptions, which is consistent with the location of the LTN at the central bright peak on the HST image.

The out-of-eclipse SED. During active phases the profile of the out-of-eclipse continuum is of the same type (here Fig. 9 and Figs. 1, 2 of Skopal et al. 1998). A relatively cool pseudophotosphere (here $T_{\mathrm{h}} \sim 14000 \mathrm{~K}$ ) is drastically affected by absorption of the veiling gas and the far-UV continuum is attenuated by a few $\times 10^{22} \mathrm{~cm}^{-2}$ hydrogen atoms. These effects are present at all orbital phases. Modeling the continuum of the 30/05/93 spectrum we neglected the nebular component of radiation due to a very low level of the continuum at $\operatorname{Ly} \alpha$. Finally, we note that including the influence of the iron curtain in modeling the ultraviolet continuum changes the interpretation suggested by Skopal et al. (1998).

Quiescent phases. All components of radiation associated with the hot star are negligible during quiescent phases (cf. Fig. 1 of Skopal et al. 1998). Such a behaviour represents a significant difference from all other symbiotic stars with relevant observations.

\subsection{Cl Cygni}

The last active phase of CICyg began in 1975 (Belyakina 1992). During the first four cycles from the maximum, narrow minima - eclipses - developed in the LC; a typical feature of active phases of symbiotic stars with a high orbital inclination. From about 1985 the profile of minima became very broad indicating a quiescent phase (Fig. 10). Accordingly we selected observations from the active phase (SWP03816 + LWR03396, 05/01/79, $\varphi=0.39$ ), from the following eclipse (SWP09255 + LWR08003, 10/06/80, $\varphi=0.0$ ) and the quiescence (SWP36321 + LWP15571, 22/05/89, $\varphi=0.82$ ).

Radiation from the giant. Fluxes corresponding to the $I J K L$ photometry can be matched by a synthetic spectrum of $T_{\text {eff }}=3300 \mathrm{~K}$ scaled with $k_{\mathrm{g}}=4.0 \times 10^{-18}\left(\theta_{\mathrm{g}}=\right.$ $2.0 \times 10^{-9}$ ), which yields the integrated flux, $F_{\mathrm{g}}^{\mathrm{obs}}=2.8 \times$ $10^{-8} \mathrm{erg} \mathrm{cm}^{-2} \mathrm{~s}^{-1}$. We note that the surface brightness relation for M-giants gives a similar value of $\theta_{\mathrm{g}}=2.1 \times 10^{-9}$ for the observed reddening-free magnitudes, $K=4.37$ and $J=5.63 \mathrm{mag}$. Timing of the eclipse profile, which yields $R_{\mathrm{g}} / A=0.38 \pm 0.02$, and other fundamental parameters derived by Kenyon et al. (1991) suggest the giant's radius $R_{\mathrm{g}} \sim 180 R_{\odot}$. This implies the distance $d \sim 2.0 \mathrm{kpc}$ and the luminosity $L_{\mathrm{g}} \sim 3400(d / 2.0 \mathrm{kpc})^{2} L_{\odot}$.
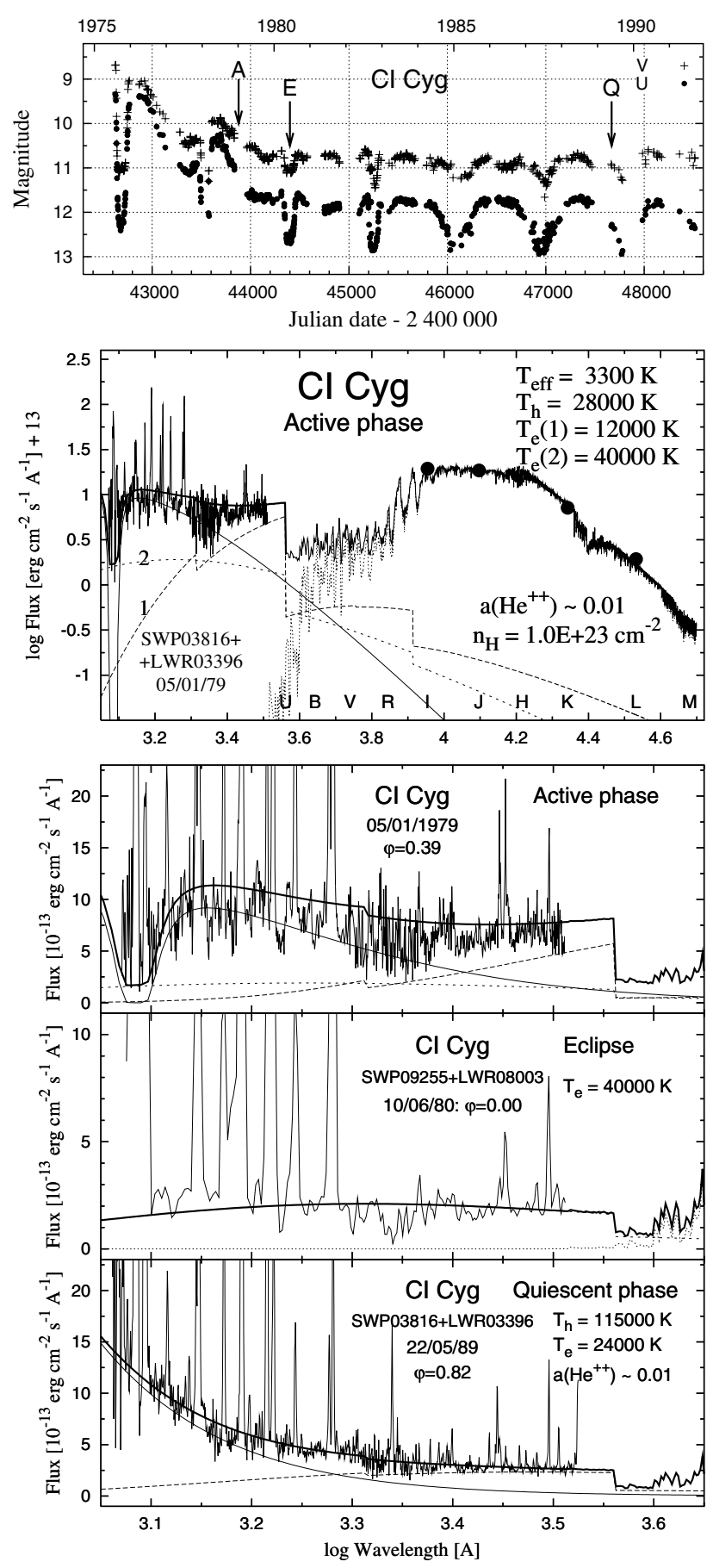

Fig. 10. Examples of the SED during the activity $(A)$, eclipse $(E)$ and quiescence $(Q)$ for CI Cyg. Corresponding dates are marked in the LC.

Radiation from the ultraviolet: active phase. The profile of the UV continuum during the active phase of CICyg is of the same type as that of other active symbiotics with a high orbital inclination - Rayleigh attenuated radiation from a warm HSS, which is affected by the iron curtain absorptions (here $T_{\mathrm{h}} \sim 28000 \mathrm{~K}, k_{\mathrm{h}} \sim 5.7 \times 10^{-23}$ and $n_{\mathrm{H}} \sim 1 \times$ $10^{23} \mathrm{~cm}^{-2}$ ). This component represents the main contribution in the UV/U spectral region. In addition, two components of the nebular radiation, from the HTN and LTN, as described for $\mathrm{Z}$ And, are required to get a satisfactory fit. The HTN emission 

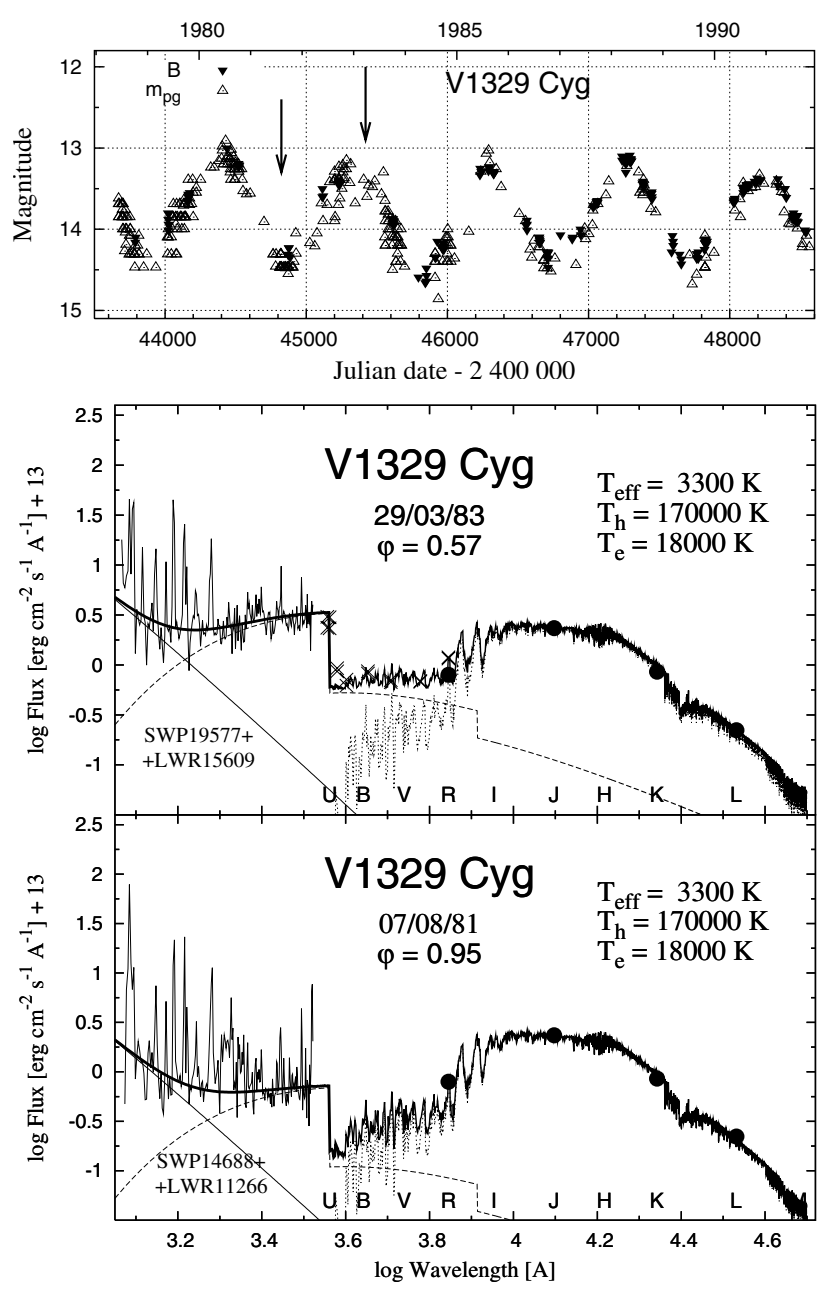

Fig. 11. The SED in the continuum of V1329 Cyg at maximum and minimum of the wave-like variation in the LC. Crosses $(\times)$ at the SED from the maximum represent continuum fluxes taken from the optical spectra presented in Figs. 192 and 193 of Munari \& Zwitter (2002). Dates of the IUE observations are marked in the LC.

is seen directly during the eclipse as the only component and out of the eclipse its presence is signaled by the non-zero level of the Rayleigh attenuated far-UV continuum (Fig. 10).

Radiation from the ultraviolet: quiescent phase. In fitting the ultraviolet continuum we fixed the hot star temperature to its Zanstra temperature, $T_{\mathrm{h}}=115000 \mathrm{~K}$ (MNSV, see Sect. 5.2.2 for details). Our solution yields a rather high electron temperature for the nebula, $T_{\mathrm{e}}=24000 \pm 4000 \mathrm{~K}$, which is constrained by a flat profile and a high level of the longwavelength part of the continuum $(\lambda>2000 \AA)$. The scaling factor of the nebular component, $k_{\mathrm{N}}=1.0 \times 10^{15} \mathrm{~cm}^{-5}$, gives $E M_{\mathrm{obs}}=4.8 \times 10^{59}(d / 2.0 \mathrm{kpc})^{2} \mathrm{~cm}^{-3}$.

\subsection{V1329 Cygni}

V1329 Cyg is a symbiotic nova which erupted in 1964 and peaked at $m_{\mathrm{pg}}=11.5 \mathrm{mag}$ in 1966 October. Prior to this outbreak, V1329 Cyg was an inactive star of about 15th magnitude displaying $\sim 2$ mag deep eclipses (see Fig. 1 of Munari et al. 1988). After the outburst the symbiotic phenomenon with characteristics of a classical symbiotic star developed. For the purpose of this paper we selected observations from the maximum (SWP19577 + LWR15609, 29/03/83, $\varphi=0.57$ ) and minimum (SWP14688 + LWR11266, 07/08/81, $\varphi=0.95$ ) of the wave-like variation in the optical LCs (Fig. 11). To better visualize their profile we smoothed them by averaging the measured points within $10 \AA$ A bins.

Radiation from the giant. Infrared fluxes were derived from average values of $R J K L$ measurements published by Tamura (1983), Lorenzetti et al. (1985), Taranova \& Yudin (1986), Munari et al. (1988), Nussbaumer \& Vogel (1991) and Munari et al. (1992). They can be matched by a synthetic spectrum of $T_{\mathrm{eff}}=3300 \mathrm{~K}$ scaled to $F_{\mathrm{g}}^{\mathrm{obs}}=3.4 \times 10^{-9} \mathrm{erg} \mathrm{cm}^{-2} \mathrm{~s}^{-1}$, $\left(k_{\mathrm{g}}=5.2 \times 10^{-19}, \theta_{\mathrm{g}}=7.2 \times 10^{-10}\right)$. The same value of $\theta_{\mathrm{g}}$ can be obtained for the M-giant's magnitudes, $K=6.67$ and $J=7.88 \mathrm{mag}$, by using the Dumm \& Schild (1998) relation. Combining these parameters with those recently derived by Fekel et al. (2001) $\left(M_{\mathrm{g}}=2.2 M_{\odot}, M_{\mathrm{h}}=0.75 M_{\odot}, R_{\mathrm{g}}=132 \pm\right.$ $40 R_{\odot}$ ) we get the distance $d=4.2 \pm 1.3 \mathrm{kpc}$, and the luminosity $L_{\mathrm{g}}=1900(d / 4.2 \mathrm{kpc})^{2} L_{\odot}$, where the uncertainty reflects only that in $R_{\mathrm{g}}$. On the other hand, the maximum width of the pre-outburst eclipses, $t_{4}-t_{1} \sim 0.13 P_{\text {orb }}$ (Fig. 1 of Schild \& Schmid 1997), yields $R_{\mathrm{g}} \sim 228 R_{\odot}$ assuming the eclipsed object to be a point source. However, such a large radius of the giant could result from an atmospheric extinction as in the case of BF Cyg (Sect. 4.8). Therefore we prefer the value of $132 R_{\odot}$, which was derived under the assumption that the giant rotates synchronously with the orbital revolution.

Quiescent phase. Radiation of V1329Cyg in the UV/optical domain is characterized by a very strong nebular emission $\left(T_{\mathrm{e}}=18000 \mathrm{~K}, k_{\mathrm{N}}=1.1 \times 10^{15} \mathrm{~cm}^{-5}\right)$, which dominates the spectrum in the range of $1660-7000 \AA$ at the maximum (Fig. 11). This constrains the very high temperature of the ionizing source, $T_{\mathrm{h}}>170000 \mathrm{~K}=T_{\mathrm{h}}^{\mathrm{min}}$ scaled to the far$\mathrm{UV}$ continuum with $k_{\mathrm{h}}=2.91 \times 10^{-25}$, to produce the observed emission measure, $E M_{\mathrm{obs}}=2.3 \times 10^{60}(d / 4.2 \mathrm{kpc})^{2} \mathrm{~cm}^{-3}$ at the maximum (i.e. to get the parameter $\delta<1$, Eq. (23)). We note that $T_{\mathrm{h}}^{\min }$ required by our model is higher then the Zanstra temperature of $145000 \mathrm{~K}$ (MNSV). At the minimum the far-UV continuum faded by a factor of about 2 as in the case of Z And (Sect. 4.3) and the nebular emission decreased by a factor of nearly 5 (Table 3 ). The latter is responsible for the large amplitude of the periodic variation in the LC.

\subsection{LT Delphini}

This yellow symbiotic star shows very pronounced orbitallyrelated brightness variation in the $U$ band. This suggests a strong and variable nebular emission in the system (Fig. 12). In 1994, Archipova et al. (1995) detected for the first time an optical outburst of this star. There is only one spectrum in the IUE archive taken by the short-wavelength prime (SWP39089, $14 / 06 / 90, \varphi=0.49)$. In spite of this we included this object in our sample to get a better representation for S-type yellow symbiotics. We determined a new value of $E_{B-V}=0.20$ by reddening $1-2 \times 10^{5} \mathrm{~K}$ Plank functions to fit the observed continuum points of the SWP spectrum. Note that the amount of the interstellar extinction at the far-UV is similar to that at $2200 \AA$. 

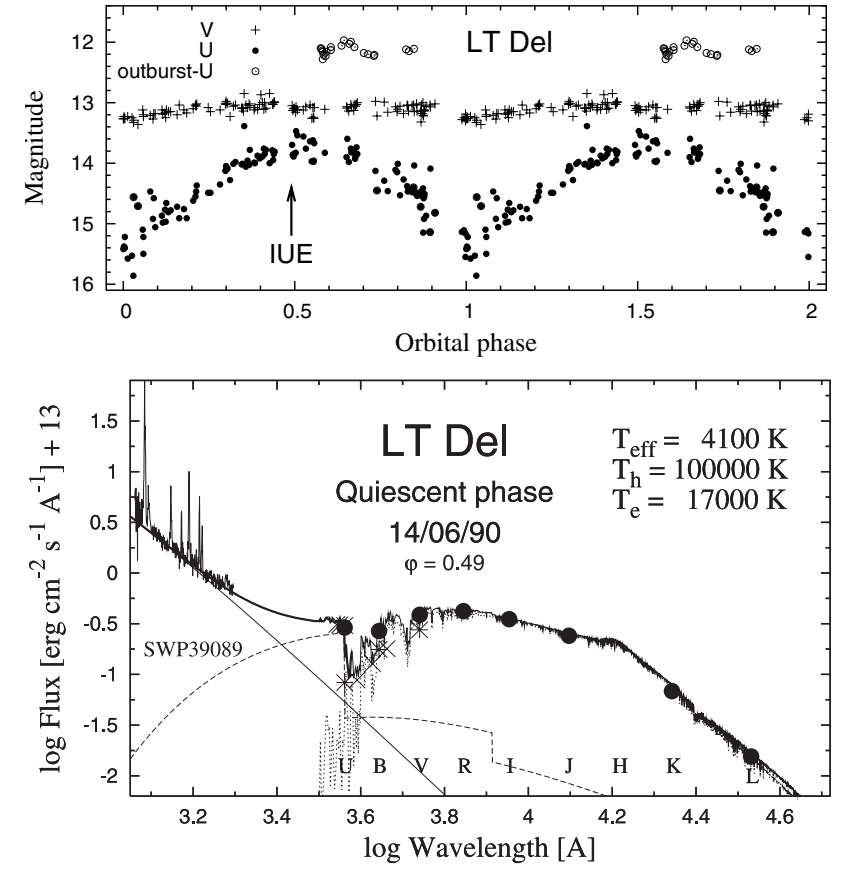

Fig. 12. Approximate SED for LT Del (LWP spectrum was not available). The SWP spectrum was taken at the maximum of the wave-like brightness variation in $U$. Full circles in the SED $(\bullet)$ are photometric fluxes from the maximum, while asterisk $(*)$ from the minimum. Crosses $(\times)$ represent continuum fluxes from the optical spectrum (Munari \& Buson 1992). Small values of the optical/IR fluxes from the giant suggest that LT Del is a rather distant object.

A previous value of $E_{B-V}=0.35$ (Munari \& Buson 1992) led to a strong enhancement of the far-UV fluxes, far beyond of any reasonable model.

Radiation from the giant. Fluxes of VRIJKL photometric measurements can be matched by a synthetic spectrum of $T_{\text {eff }}=4100 \mathrm{~K}$ and scaling of $k_{\mathrm{g}}=3.0 \times 10^{-20}$, which yields the bolometric flux, $F_{\mathrm{g}}^{\mathrm{obs}}=4.8 \times 10^{-10} \mathrm{erg} \mathrm{cm}^{-2} \mathrm{~s}^{-1}$. Our value of $T_{\text {eff }}$ is markedly lower than that suggested by the current spectral classification as K0 (Mürset \& Schmid 1999). With respect to this disagreement we note that spectral classification of yellow symbiotics is based on features of $\mathrm{G}$ band, CaI $\lambda 4227, \mathrm{Fe} I \lambda 4405$ and absorptions of Fe I, SrII and the CH band. Mürset \& Schmid (1999) pointed out that this approach may be inaccurate for stars with peculiar abundance patterns (here AG Dra, LT Del, BD - $21^{\circ} 3873$ ). Our effective temperature from IR fluxes suggests a later spectral type, K3-K4, of a normal giant (Belle et al. 1999). Consequently we adopted the corresponding average giant's radius of $R_{\mathrm{g}} \sim 30 R_{\odot}$, which gives the distance $d=R_{\mathrm{g}} / \theta_{\mathrm{g}} \sim 3.9 \mathrm{kpc}$ and the luminosity $L_{\mathrm{g}}=230(d / 3.9 \mathrm{kpc})^{2} L_{\odot}$. We note that the giant's radius of $30 R_{\odot}$ is well inside the range of 18 to $36\left(M_{\mathrm{g}} / 1.5 M_{\odot}\right)^{1 / 2} R_{\odot}$, which can be derived from atmospheric $\log (g)=1.8 \pm 0.3$ (Pereira et al. 1998).

Radiation from the ultraviolet. Having only SWP spectrum and a few flux-points from the simultaneous optical spectroscopy (Munari \& Buson 1992) the resulting model is approximate, mainly in $T_{\mathrm{e}}$ with an uncertainty of $\pm 3000 \mathrm{~K}$. $T_{\mathrm{h}}=100000 \mathrm{~K}$ was adopted as a characteristic temperature. The model is shown in Fig. 12.

\subsection{AG Draconis}

The LC of AG Dra shows numerous brightenings by 2-3 mag in $U$ with maxima often separated approximately by 1 year. Recently, two major eruptions were recorded during 1981-83 and 1994-96 accompanied by many short-term events lasting from a few weeks to a few months. González-Riestra et al. (1999) identified cool (1981-83 and 1994-96) and hot (e.g. 1985-86) outbursts differing in their Zanstra temperatures. The quiescent LC is characterized by a periodic wavelike variation. The top panel of Fig. 13 demonstrates these characteristics. The data are from Skopal et al. (2004) and Leedjärv et al. (2004). There are no signs either in the optical or farUV regions of eclipses. Schmid \& Schild (1997) derived the orbital inclination $i=60\left( \pm 8^{\circ} .2\right)$. To demonstrate the nature of the observed light variation we selected two IUE spectra from quiescence taken at different spectroscopic conjunctions of the binary components (SWP37473 + LWP16675, 27/10/89, $\varphi=0.62 ;$ SWP06650 + LWR05691, 25/09/79, $\varphi=0.91)$ and two spectra from active phases; one from the major $1994 \mathrm{cool}$ outburst (SWP51632 + LWP28752, 28/07/94) and one from the hot 1985 eruption (SWP25443 + LWP05513, 13/03/85).

Radiation from the giant. VRI measurements from the light minima during quiescence (recently Leedjärv et al. 2004) and the infrared JHKLM photometry of AGDra can be matched by synthetic spectra with $T_{\text {eff }}=4100-4300 \mathrm{~K}$. According to the analysis of Smith et al. (1996) we adopted here $T_{\text {eff }}=$ $4300 \mathrm{~K}$. The scaling factor of this spectrum, $k_{\mathrm{g}}=4.9 \times$ $10^{-19}$, corresponds to the bolometric flux $F_{\mathrm{g}}^{\mathrm{obs}}=9.51 \times$ $10^{-9} \mathrm{erg} \mathrm{cm}^{-2} \mathrm{~s}^{-1}$. From the surface gravity, $\log (g)=1.6 \pm$ 0.3 (Smith et al. 1996), one can derive the giant's radius as $R_{\mathrm{g}}=(33 \pm 11)\left(M_{\mathrm{g}} / 1.5 M_{\odot}\right)^{1 / 2} R_{\odot}$ and the distance $d=$ $R_{\mathrm{g}} / \theta_{\mathrm{g}}=(1.1 \pm 0.4)\left(M_{\mathrm{g}} / 1.5 M_{\odot}\right)^{1 / 2} \mathrm{kpc}$, where the giant's mass of $1.5 M_{\odot}$ was taken from Mikolajewska et al. (1995). The giant's luminosity $L_{\mathrm{g}}=360(d / 1.1 \mathrm{kpc})^{2} L_{\odot}$.

Radiation from the ultraviolet: quiescent phase. The SED of AG Dra during quiescent phase is characterized by (i) a dominant giant's radiation in the optical, which results in large differences between amplitudes of the orbitally-related variation ( $\Delta U \gg \Delta B \gtrsim \Delta V$ ); (ii) a strong nebula radiating at a high temperature $\left(T_{\mathrm{e}} \gtrsim 20000 \mathrm{~K}\right)$ and varying in its $E M$ along the orbit; and (iii) a stable hot stellar component of radiation, which is contrary to the eclipsing systems. Fitting the spectrum from the maximum $(27 / 10 / 89)$ we found the lower limit of the hot star temperature, $T_{\mathrm{h}}^{\min }=110000 \mathrm{~K}\left(k_{\mathrm{h}}=5.4 \times 10^{-25}\right)$, to satisfy the ionization condition given here through the parameter $\delta=1$ (Sect. 4.1, Eq. (23)). These parameters determine the lower limit of the hot star's ionizing capacity to produce the observed emission measure in maximum given by the fitting parameters $k_{\mathrm{N}}=5.5 \times 10^{14} \mathrm{~cm}^{-5}$ and $T_{\mathrm{e}}=21500 \mathrm{~K}$.

Radiation from the ultraviolet: active phase. The SED during the major 1994 outburst is dominated by the nebular radiation at a high electron temperature, $T_{\mathrm{e}}=35000 \pm 5000 \mathrm{~K}$ $\left(k_{\mathrm{N}}=2.1 \pm 0.4 \times 10^{16} \mathrm{~cm}^{-5}\right)$ which determines the continuum profile in the ultraviolet and significantly affects the optical with a large contribution in the infrared. At $6 \mathrm{~cm}$ radio wavelengths, Ogley et al. (2002) measured a total emission of $\sim 1$ mJy from AGDra in 2000 March. They suggested a 

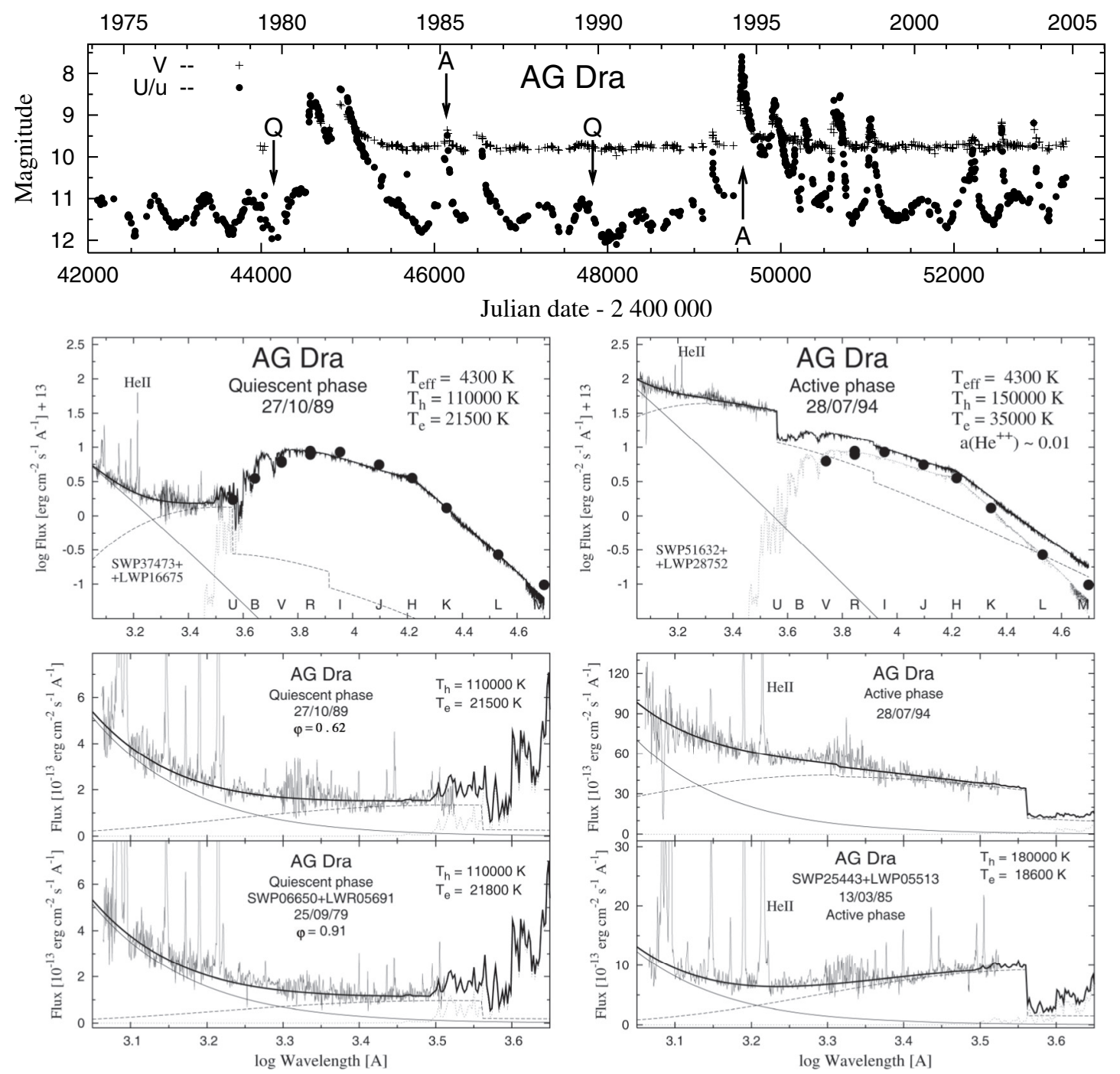

Fig. 13. The SED of AGDra during quiescence (left panels) and active phases (right). Note the variation of the nebular emission and, in contrast, the stability of the hot stellar component with the orbital phase during quiescence. The SED during the active phases is characterized by a significant increase of the nebular emission, which constrains very high temperature of the ionizing source. Dates of the IUE observations are marked in the U/V LCs (top).

nebular nature for this emission. Also during the smaller eruptions (e.g. 1985, 1986) the nebular emission represents a significant component of radiation in the UV spectrum (right panels of Fig. 13). Here we present the case of the 1985 eruption. We estimated the lower limits for the temperature of the ionizing source, $T_{\mathrm{h}}^{\min }=150000 \mathrm{~K}$ and $180000 \mathrm{~K}$, for the major 1994 "cool" outburst and the "hot" 1985 eruption, respectively.

\subsection{CQDraconis}

Reimers et al. (1988) discovered a UV bright companion of the M 3 III giant 4Dra, determined elements of the spectroscopic orbit of the giant and suggested that the variation in the UV could be produced by a CV of an AMHer-type. Recently, Wheatley et al. (2003), based on their X-ray observation, suggested that CQDra is most likely a symbiotic binary containing a white dwarf accreting material from the wind of the red giant. Here we selected IUE observations from two dates (SWP24265 + LWP04630, 23/10/84, $\varphi=0.80$ and SWP29421 + LWP09295, 11/10/86, $\varphi=0.22$ ) to verify if the observed properties of the UV continuum are consistent with the ionization model of symbiotic binaries.

Radiation from the giant. The red giant in CQ Dra is a dominant source of radiation in the optical/IR wavelengths (Fig. 14). Therefore we used also average values of the $U B V$ measurements ( $U=8.35, B=6.6$ and $V=5.0 \mathrm{mag}$ ) together with the $J H K$ photometry of Kamath \& Ashok (1999) to select an appropriate synthetic spectrum. In addition, we tried to match an "emission" bump from the giant at $\sim \lambda 3200 \AA \mathrm{ob}-$ served by the IUE. In this manner we selected the synthetic spectrum of $T_{\text {eff }}=3700 \mathrm{~K}$ and determined the scaling factor $k_{\mathrm{g}}=1.5 \times 10^{-16}$, which yields the observed bolometric flux $F_{\mathrm{g}}^{\mathrm{obs}}=1.6 \times 10^{-6} \mathrm{erg} \mathrm{cm}^{-2} \mathrm{~s}^{-1}$. For the HIPPARCOS distance of $178 \pm 15$ pc (Perryman et al. 1997) the giant's radius $R_{\mathrm{g}}=d \times \theta_{\mathrm{g}}=95(d / 178 \mathrm{pc}) R_{\odot}$ and its luminosity, $L_{\mathrm{g}}=1600(d / 178 \mathrm{pc})^{2} L_{\odot}$. 

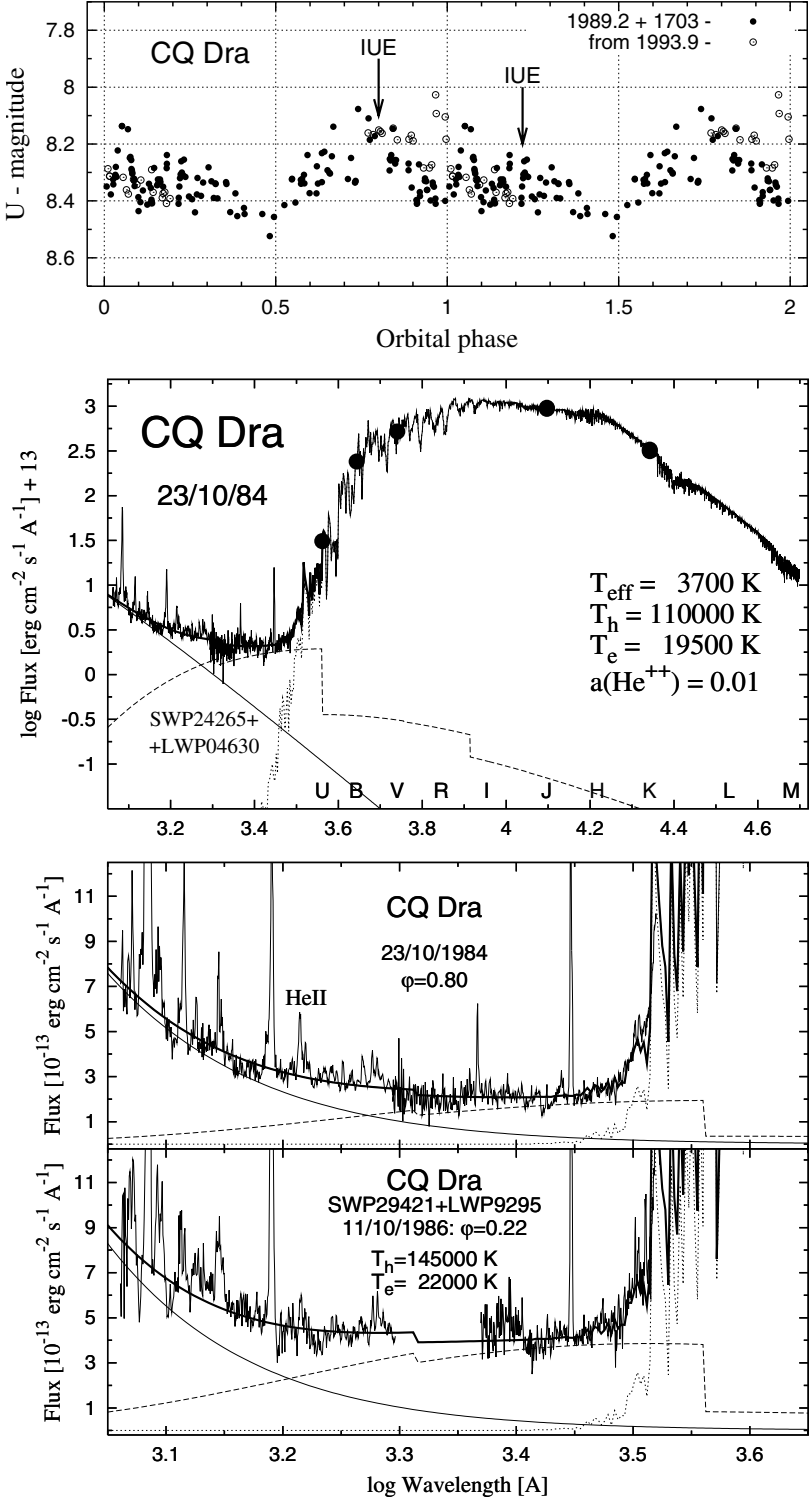

Fig. 14. The SED of CQDra from two shifts of the IUE satellite. A strong and variable nebular emission dominates the ultraviolet while in the optical/IR we can detect radiation from only the red giant. Variation in the $U$ band reflects probably that in the nebular radiation. Optical photometry is from Hric et al. (1994).

Radiation from the ultraviolet. The profile of the ultraviolet continuum with the superposed emission features is typical of a symbiotic binary during quiescent phase. The continuum is nearly flat between approximately 1600 to $3200 \AA$, which suggests a dominant contribution from the nebula radiating at a high electron temperature. This constrains a high temperature for the hot stellar radiation to produce the observed nebular emission. In addition, the two selected spectra show a variation in the emission measure by a factor larger than 2 , while the scaling of the hot stellar radiation persists practically unchanged. This implies an increase of the hot star temperature from about $T_{\mathrm{h}}^{\mathrm{min}}=110000 \mathrm{~K}$ to about $145000 \mathrm{~K}$. Parameters of our solution (a low luminosity, $L_{\mathrm{h}}=6.6-14(d / 178 \mathrm{pc})^{2} L_{\odot}$ and the effective radius, $\left.R_{\mathrm{h}}^{\text {eff }}=0.007-0.006(d / 178 \mathrm{pc}) R_{\odot}\right)$ suggest that the sole source of the energy detected from the ultraviolet is accretion from the giant's wind at the rate of $\dot{M}_{\text {acc }}=$ a few $\times 10^{-9} M_{\odot} \mathrm{yr}^{-1}$ for the mass of a white dwarf, $M_{\mathrm{WD}} \equiv 1.0 M_{\odot}$ (Hamada \& Salpeter 1961). According to description on wind accretion onto white dwarfs (Livio \& Warner 1984) these accretion rates require the mass-loss rate from the giant $\dot{M}_{\mathrm{W}} \lesssim 10^{-7} M_{\odot} \mathrm{yr}^{-1}$ for the separation of the binary components at times of observations, 750 and $900 R_{\odot}$ $\left(M_{\mathrm{T}} \equiv 2.5 M_{\odot}\right.$ and elements from Reimers et al. 1988). Then an increase in $\dot{M}_{\mathrm{W}}$ gives a larger $\dot{M}_{\text {acc }}$ and a higher temperature of the ionizing source (inner parts of an accretion disk), which causes an increase in the emission measure, because a larger fraction of the giant's wind can be ionized. The first results of studying the accretion process in CQ Dra are discussed in more detail by Skopal (2005).

\subsection{V443 Herculis}

V443 Her is a quiet symbiotic star - no outburst has been recorded to date. The LC displays a marked wave-like variation of the optical light along the orbital phase (Fig. 15). Recent studies of this symbiotic (Dobrzycka et al. 1993; Kolotilov et al. 1995; Skopal 1996) suggested a low orbital inclination $\left(\approx 30^{\circ}\right.$, and $18^{\circ}$, respectively). We selected two pairs of IUE spectra taken at the maximum of the light (SWP10439+ LWR09118, 20/10/80, $\varphi=0.50$ ) and at the quadrature $($ SWP42772 + LWP21540, 20/10/91, $\varphi=0.25)$. No wellexposed spectrum at/around the minimum was available.

Radiation from the giant. The JKLM photometry (Kolotilov \& Yudin 1994) and a flat optical BVR continuum suggest a low effective temperature for the giant's photosphere (Fig. 15). A synthetic spectrum of $T_{\text {eff }}=3300 \mathrm{~K}$ scaled to $F_{\mathrm{g}}^{\mathrm{obs}}=$ $1.19 \times 10^{-8} \mathrm{erg} \mathrm{cm}^{-2} \mathrm{~s}^{-1}\left(k_{\mathrm{g}}=1.8 \times 10^{-18}, \theta_{\mathrm{g}}=1.33 \times\right.$ $10^{-9}$ ) satisfies best the photometric measurements. Also the surface brightness relation for M-giants gives a very close value of $\theta_{\mathrm{g}}=1.37 \times 10^{-9}$ for the reddening-free magnitudes, $K=5.32$ and $J=6.56 \mathrm{mag}$. As there is no reliable estimate of the giant's radius in the literature we adopted $R_{\mathrm{g}} \sim 130 R_{\odot}$ according to the empirical relations between the radius and $T_{\text {eff }}$ for M-giants (Belle et al. 1999). Then the distance $d=$ $R_{\mathrm{g}} / \theta_{\mathrm{g}} \sim 2.2\left(R_{\mathrm{g}} / 130 R_{\odot}\right) \mathrm{kpc}$ and the luminosity $L_{\mathrm{g}} \sim$ $1800(d / 2.2 \mathrm{kpc})^{2} L_{\odot}$.

Radiation from the ultraviolet. The stellar component of the radiation represents a dominant contribution in this spectral region. It has a relatively small slope in the far-UV and rivals other components of radiation up to the $B$ band (Fig. 15). Our model of the $21 / 10 / 80$ spectrum suggests a low temperature, $T_{\mathrm{h}}=39000+11000 /-5000 \mathrm{~K}$, where uncertainties were determined in the same way as in Skopal (2001b). Both the spectra require the same scaling, $k_{\mathrm{h}}=2.32 \times 10^{-23}$ $\left(\theta_{\mathrm{h}}=4.82 \times 10^{-12}\right)$, which corresponds to the effective radius $R_{\mathrm{h}}^{\text {eff }}=0.47(d / 2.2 \mathrm{kpc}) R_{\odot}$ and the luminosity $L_{\mathrm{h}}=$ $460(d / 2.2 \mathrm{kpc})^{2} L_{\odot}$. On the other hand, the nebular emission is faint and varies along the orbit: $k_{\mathrm{N}}=1.0$ and $0.4 \times 10^{15} \mathrm{~cm}^{-5}$ at $\varphi=0.5\left(T_{\mathrm{e}}=15500 \pm 3000 \mathrm{~K}\right)$ and $\varphi=0.25\left(T_{\mathrm{e}}=12000 \pm\right.$ $2000 \mathrm{~K})$, respectively. However, the corresponding parameter $\delta=3.9$ and 2.0 is not consistent with the fitting parameters $T_{\mathrm{h}}$, $k_{\mathrm{h}}$ and $k_{\mathrm{N}}$. The observed hot stellar radiation is not capable of 

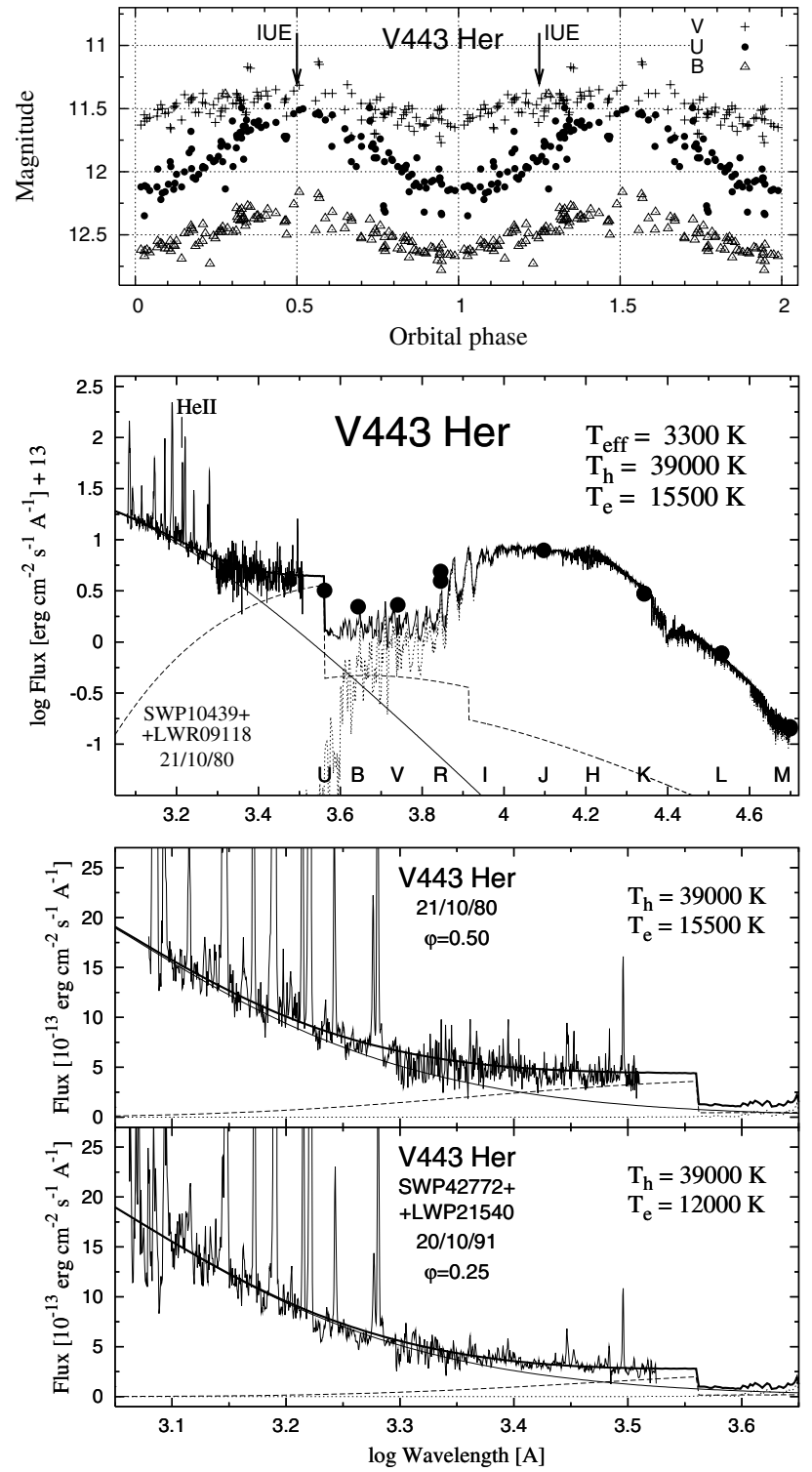

Fig. 15. The SED of V443 Her at two different positions of the binary (marked in the LC top). The nebular component of radiation varies with the orbital phase, while the hot stellar radiation is stable.

producing the nebular emission, because of the too low temperature given by the fit $\left(T_{\mathrm{h}} \ll T_{\mathrm{h}}^{\min } \sim 75000\right)$. This conflicting situation signals that an unseen part of the ionizing source has to radiate at a higher temperature to produce a surplus of the nebular flux in addition to that generated only by its observed portion. Qualitatively, this could be understandable if the accreted matter has a disk-like structure, whose outer rim occults the central hot core in the direction to the observer. However, this would suggest a rather high inclination of the V443 Her binary orbit. Parameters $L_{\mathrm{h}}$ and $R_{\mathrm{h}}^{\text {eff }}$ derived from our models represent the lower and upper limits of their real values. Therefore in Table 3 we also present a formal solution for $T_{\mathrm{h}}=T_{\mathrm{h}}^{\min }=$ $75000 \mathrm{~K}$ (Sect. 4.1, Eq. (23)).
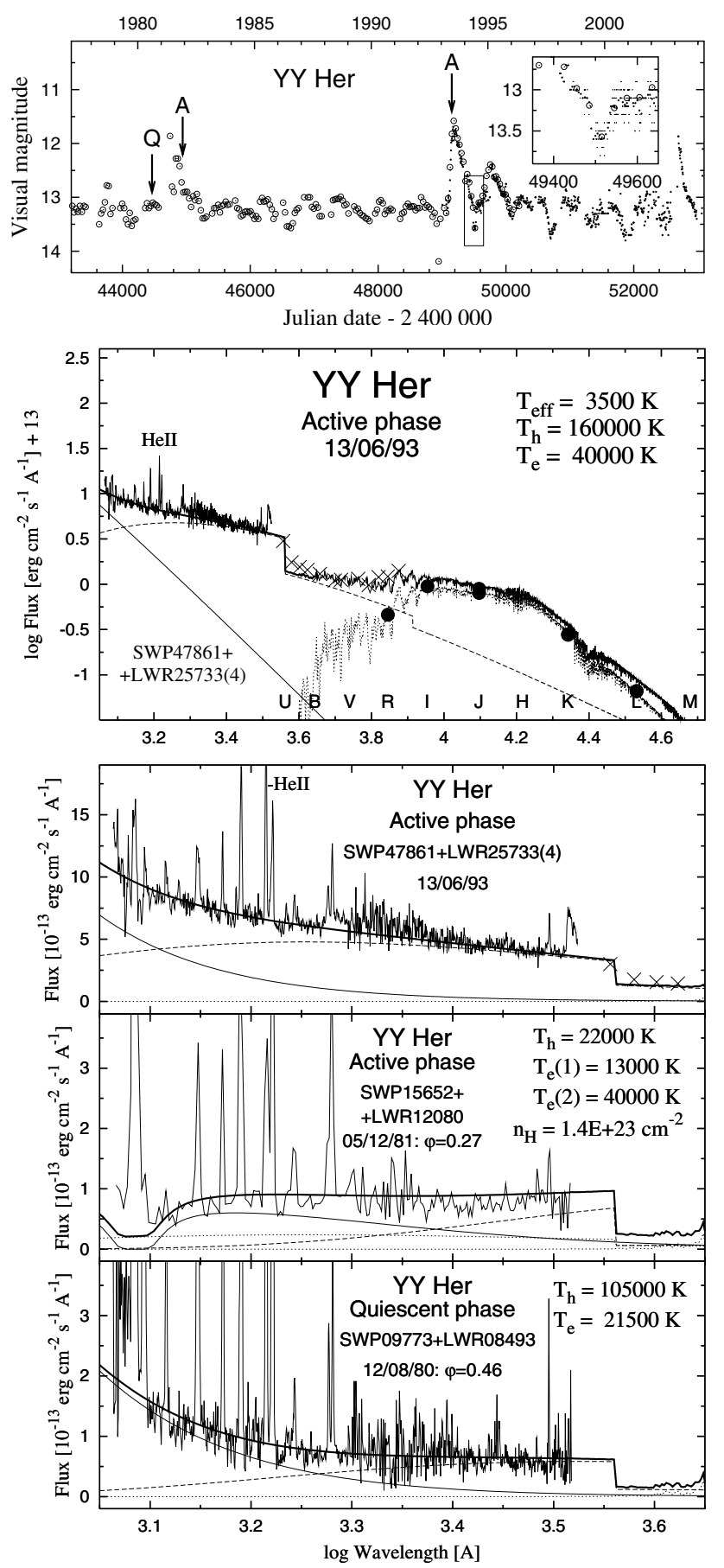

Fig. 16. The SED of YYHer during quiescence and two different phases of activity. Visual LC is composed from the data of Munari et al. (1997a) (॰) and those available from the CDS database $(\bullet)$. The eclipse effect observed in 1994.43 is shown in detail. Crosses $(\times)$ in the SED represent continuum flux-points from the optical spectrum taken on 10/06/93 (Munari et al. 1997a).

\subsection{YYHerculis}

Munari et al. (1997b) demonstrated that the LC of YY Her underwent four main outbursts and a few bright stages. Photometric measurements made after the main 1993 outburst (Hric et al. 2001; Mikolajewska et al. 2002a) revealed a relatively deeper and narrower primary minima than prior to 
the outburst (Fig. 16), which suggests a high inclination of the orbital plane. In addition, a secondary minimum in the VRI LCs appeared (Hric et al. 2001). Such evolution is similar to that of AXPer during the transition from its 1989 outburst to quiescence (Fig. 3 of Skopal et al. 2001a, Fig. 21 here). We selected IUE spectra from the maximum of the 1993 outburst (SWP47861 + LWP25733(4), 13/06/93, $\varphi=$ 0.37), a bright phase in 1981 (SWP15652 + LWR12080, $05 / 12 / 81, \varphi=0.27$ ) and quiescence (SWP09773 + LWR08493, $12 / 08 / 80, \varphi=0.46)$.

Radiation from the giant. Relatively faint RIJKL magnitudes of a normal giant (e.g. Mürset \& Schmid 1999) suggest that YYHer is a distant object. The corresponding synthetic spectrum is characterized by $T_{\text {eff }}=3500 \mathrm{~K}$ and $\theta_{\mathrm{g}}=$

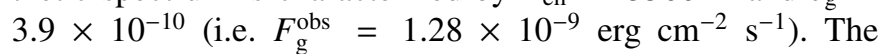
average reddening-free values of $J=8.98$ and $K=7.89$ provide nearly identical angular radius, $\theta_{\mathrm{g}}=4.0 \times 10^{-10}$ (Dumm \& Schild 1998). We estimated the radius of the giant from the profile of the 1994 minimum in the smoothed visual LC (Fig. 16). This can be interpreted in terms of the eclipse of the hot star pseudophotosphere by the giant. Its timing $\left(t_{1} \sim \mathrm{JD} 2449486, t_{2} \sim \mathrm{JD} 2449492, t_{3} \sim \mathrm{JD} 2449522\right.$, $t_{4} \sim$ JD $2449537: \pm 2-3$ days) determines the linear size of the giant's stellar disk that eclipses the object, $R_{\mathrm{g}}^{\mathrm{E}}=0.21 \mathrm{~A}$. This parameter can be expressed through the radius of the giant, $R_{\mathrm{g}}$, and the orbital inclination $i$ as

$R_{\mathrm{g}}^{\mathrm{E}} / A=\sqrt{\left(R_{\mathrm{g}} / A\right)^{2}-\cos ^{2}(i)}$.

With the aid of the empirical relations between the radius and effective temperature for giant stars (Belle et al. 1999) we adopted $R_{\mathrm{g}} \sim 110 R_{\odot}$, which satisfies Eq. (25) for $i=80^{\circ}$ and $A=403 R_{\odot}\left(M_{\mathrm{T}} \equiv 2.5 M_{\odot}\right)$. This yields the distance $d=6.3\left(R_{\mathrm{g}} / 110 R_{\odot}\right) \mathrm{kpc}$ and the giant's luminosity $L_{\mathrm{g}}=$ $1600(d / 6.3 \mathrm{kpc})^{2} L_{\odot}$. On the other hand, based on the presence of a secondary minimum in the VRI LCs, Mikolajewska et al. (2002a) suggested a Roche-lobe filling giant in the system, which corresponds to the distance $d=10 \pm 3 \mathrm{kpc}$. However, the $I$ LC shows rather narrow minima (see Fig. 2 of Hric et al. 2001) than can be ascribed to the wave-like variation due to the tidal distortion of the giant. Also a statistical approach of Mürset \& Schmid (1999) suggests the giant in YY Her to be well inside its Roche lobe.

Radiation from the ultraviolet: quiescent phase. Radiation of YY Her during quiescence comprises a hot stellar component of radiation of $T_{\mathrm{h}}>105000 \mathrm{~K}=T_{\mathrm{h}}^{\mathrm{min}}$, the angular radius $\theta_{\mathrm{h}}<4.9 \times 10^{-13}$ and the nebular component of radiation characterized by $T_{\mathrm{e}}=21500 \pm 3000 \mathrm{~K}$ and $k_{\mathrm{N}}=2.4 \times 10^{14} \mathrm{~cm}^{-5}$. Other derived parameters are given in Table 3.

Radiation from the ultraviolet: active phases. YY Her is the only case in our sample of objects that shows a very different continuum profile during active phases. During the maximum of the star's brightness (1993) the profile of the SED is of the same type as observed for AG Dra (cf. Fig. 13), while during the 1981 bright phase it was similar to that of all other active symbiotics with a high orbital inclination (e.g. CICyg). In the former case the SED from the ultraviolet to the near-IR region is dominated by the nebular radiation at a high electron temperature $\left(T_{\mathrm{e}} \sim 40000 \mathrm{~K}, k_{\mathrm{N}}=2.7 \times 10^{15} \mathrm{~cm}^{-5}\right)$, which is powered by a strong stellar source radiating at $T_{\mathrm{h}}>$ $160000 \mathrm{~K}=T_{\mathrm{h}}^{\min }$ and scaled with $\theta_{\mathrm{h}}<6.3 \times 10^{-13}$ (i.e. $\delta<1$ ). The profile of the simultaneously observed optical spectrum (Munari et al. 1997a) agrees well with the modeled SED (Fig. 16). In the latter case the UV continuum results from superposition of radiation from a cool stellar pseudophotosphere $\left(T_{\mathrm{h}}=22000 \mathrm{~K}, \theta_{\mathrm{h}}=3.2 \times 10^{-12}\right)$ attenuated with $n_{\mathrm{H}} \sim$ $1.4 \times 10^{23} \mathrm{~cm}^{-2}$, a low-temperature nebula $\left(T_{\mathrm{e}}=13000 \mathrm{~K}\right.$, $\left.k_{\mathrm{N}}=1.5 \times 10^{14} \mathrm{~cm}^{-5}\right)$ and a high-temperature nebula $\left(T_{\mathrm{e}} \equiv\right.$ $40000 \mathrm{~K}, k_{\mathrm{N}}=1.3 \times 10^{14} \mathrm{~cm}^{-5}$ ).

\subsection{RW Hydrae}

RW Hya is a stable symbiotic system for which no optical eruption has been observed. Its LC displays a wave-like variation as a function of the orbital phase (Fig. 17). A high inclination of the orbital plane is indicated by the eclipse effect due to attenuation of the far-UV continuum by Rayleigh scattering (e.g. Schild et al. 1996). IUE spectra of RW Hya have been studied in detail by many authors (Kenyon \& Mikolajewska 1995; Schild et al. 1996; Dumm et al. 1999; Sion et al. 2002). We selected one observation around the maximum $($ SWP05863 + LWR05110, 19/07/79, $\varphi=0.32)$ and one around the minimum of the optical light (SWP31039+ LWP10825, 24/05/87, $\varphi=0.06$ ) to demonstrate our approach for this case.

Radiation from the giant. A synthetic spectrum of $T_{\mathrm{eff}}=$ $3800 \mathrm{~K}$ with scaling of $k_{\mathrm{g}}=2.6 \times 10^{-18}\left(\theta_{\mathrm{g}}=1.61 \times 10^{-9}\right.$, $\left.F_{\mathrm{g}}^{\mathrm{obs}}=3.10 \times 10^{-8} \mathrm{erg} \mathrm{cm}^{-2} \mathrm{~s}^{-1}\right)$ matches well the flux-points corresponding to the BVRIJHKL photometry. Reddening-free magnitudes, $K=4.65$ and $J=5.72 \mathrm{mag}$, also give a similar value of $\theta_{\mathrm{g}}=1.77 \times 10^{-9}$ (Dumm \& Schild 1998). The giant dominates the spectrum from the $B$ band to the infrared. This results in a large amplitude difference of the wave-like variation at different passbands $(\Delta U \gg \Delta V$, Fig. 17). Thus, the nebular emission, which is responsible for this type of variability, is not able to rival the giant's contribution from $B$ to longer wavelengths. Assuming the synchronous rotation of the giant with the orbital revolution, Schild et al. (1996) determined its radius to $R_{\mathrm{g}}=58.5 \pm 8 R_{\odot}$. This quantity then yields the distance $d=R_{\mathrm{g}} / \theta_{\mathrm{g}}=820 \pm 112\left(R_{\mathrm{g}} / 58.5 R_{\odot}\right) \mathrm{pc}$ and the luminosity, $L_{\mathrm{g}}=650 \pm 180(d / 0.82 \mathrm{kpc})^{2} L_{\odot}$, where uncertainty in only $R_{\mathrm{g}}$ is included. These parameters are similar to those determined by Schild et al. (1996).

Radiation from the ultraviolet. A steep far-UV continuum (practically within the SWP spectrum) reflects a dominant contribution from the hot stellar source in this region. On the other hand, a flat near-UV continuum of the LWR spectrum signals a significant nebular radiation here - an illustrative example of a quiescent symbiotic's UV spectrum. Our best fit of the $19 / 07 / 79$ spectrum corresponds to $T_{\mathrm{h}}=110000 \pm 30000 \mathrm{~K}$, $k_{\mathrm{h}}=6.3 \times 10^{-24}$ and $T_{\mathrm{e}}=15000 \pm 2000 \mathrm{~K}$ with scaling, $k_{\mathrm{N}}=2.8 \times 10^{15} \mathrm{~cm}^{-5}$. This solution gives the parameter $\delta=0.54$, which means that a fraction of ionizing photons is not converted to the nebular radiation. In the sense of the STB model the ionized zone is open. The situation in which 

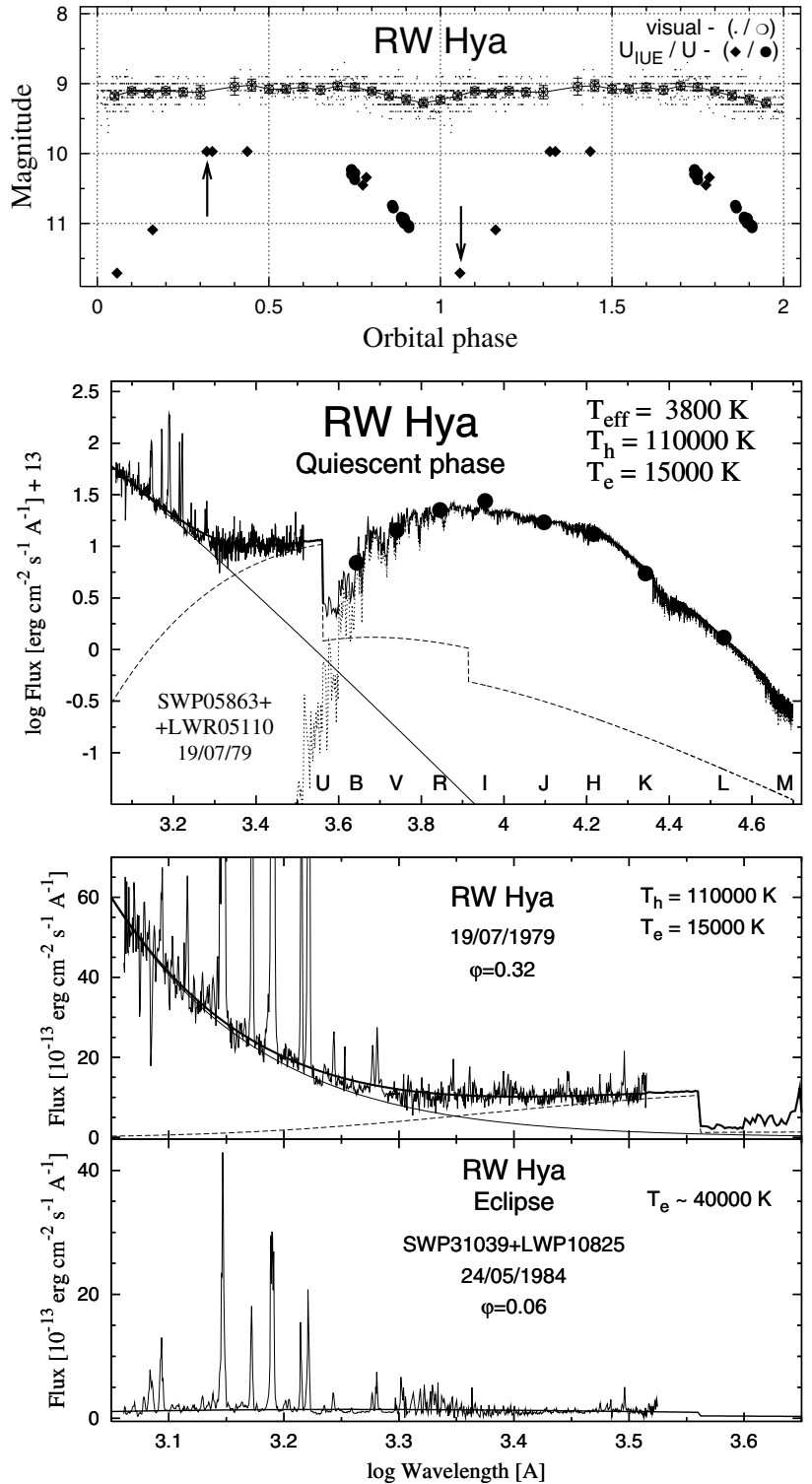

Fig. 17. Top: visual and $U$ LCs of RW Hya (Skopal et al. 2002b) with added $U$-magnitudes estimated from available IUE observations. The arrow marks position of the used spectrum. Middle and bottom: the SED near to the optical maximum shows typical characteristics of a quiescent phase with a high luminosity of the hot object, while during the eclipse only a very faint high-temperature nebular emission can be recognized.

all the $L_{\mathrm{ph}}$ photons are involved in the ionization process corresponds to $T_{\mathrm{h}}^{\mathrm{min}}=80000 \mathrm{~K}$, which represents the lower limit of the hot source temperature in RW Hya. It is also close to the Zanstra temperature of $75000 \mathrm{~K}$ derived by MNSV. The spectra of RW Hya taken close to the giant's inferior conjunction suffer an additional (to the Rayleigh scattering) wavelengthindependent attenuation (Dumm et al. 1999) as observed for other symbiotics with a high orbital inclination. During the totality only a high-temperature nebula can be recognized.
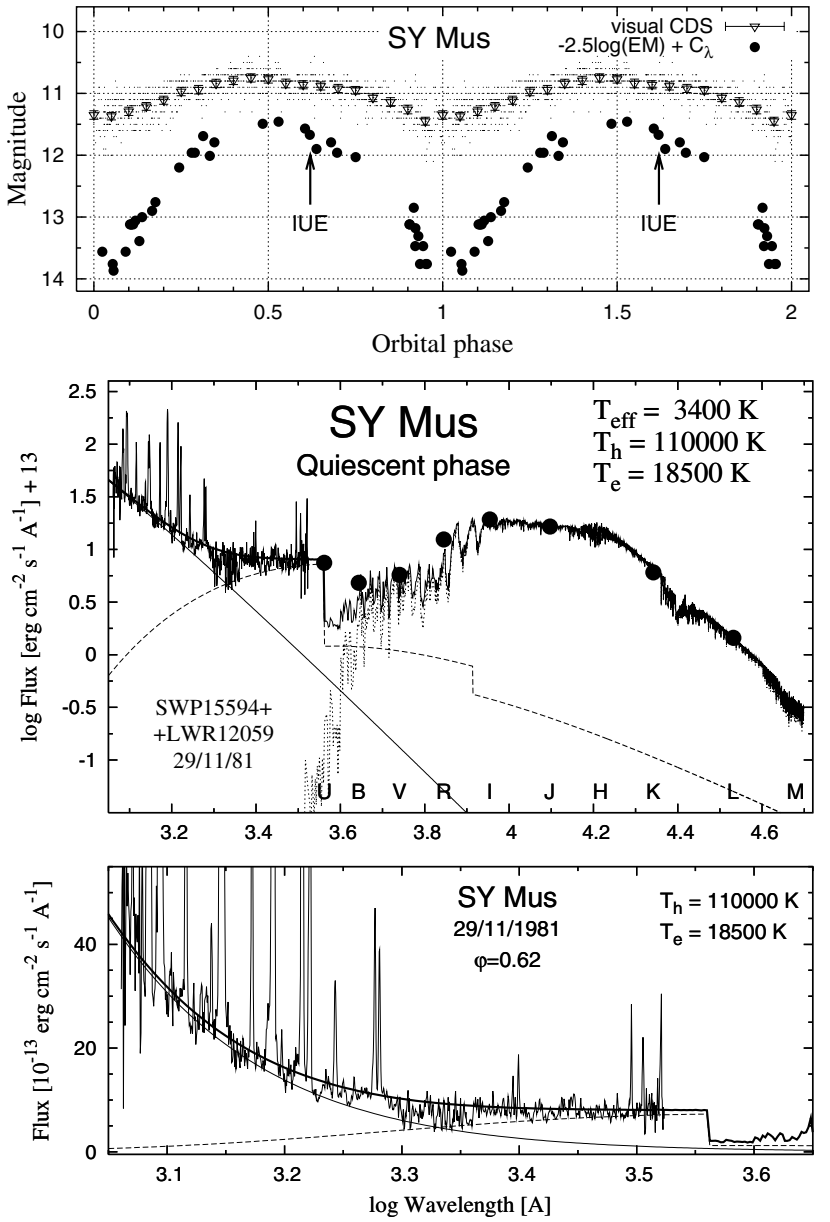

Fig. 18. Top: visual and $U$ LC of SY Mus. The former was compiled from visual estimates available at the CDS database. The latter was reconstructed from the IUE low-resolution spectra by the same way as suggested by Skopal (2001a). Middle and bottom: observed and reconstructed SEDs at the position near to its optical maximum.

\subsection{SYMuscae}

SY Mus is another stable system with a high luminosity of the hot component. Its visual LC displays a strictly periodic wavelike variation with the orbital phase (e.g. Pereira et al. 1995). Figure 18 also shows a variation in the emission measure along the orbital phase scaled to the effective wavelength of the $U$ band as suggested by Skopal (2001a). The emission measure was estimated from the near-UV, where the nebula represents a dominant source of radiation (Eq. (18), $T_{\mathrm{e}} \equiv 18500 \mathrm{~K}$ ). The system is eclipsing. When the binary approaches the position of the inferior giant's conjunction, the Rayleigh scattering significantly affects the far-UV radiation (e.g. Pereira et al. 1995). Near to the conjunction $(\varphi=0 \pm \sim 0.05)$ the UV continuum is practically flat and at the level of $1-2 \times 10^{-13} \mathrm{erg} \mathrm{cm}^{-2} \mathrm{~s}^{-1} \AA^{-1}$. Also in this case an additional wavelength-independent continuum attenuation can be recognized (Table 2 of Dumm et al. 1999). As a result we selected only one observation exposed at the position near to the maximum, where the effect of the additional opacity source can be assumed to be minimal (SWP15594 + LWR12059, 29/11/81, $\varphi=0.62)$. 
Radiation from the giant. In good agreement with the spectral classification of the giant in SY Mus (M4.5 III, Mürset \& Schmid 1999) a synthetic spectrum of $T_{\text {eff }}=3400 \mathrm{~K}$ matches well photometric measurements in the IR. Its scaling, $k_{\mathrm{g}}=$ $3.6 \times 10^{-18},\left(\theta_{\mathrm{g}}=1.9 \times 10^{-9}\right)$ corresponds to the bolometric flux, $F_{\mathrm{g}}^{\text {obs }}=2.6 \times 10^{-8} \mathrm{erg} \mathrm{cm}^{-2} \mathrm{~s}^{-1}$. According to the surface brightness relation for M-giants (Dumm \& Schild 1998), the reddening-free magnitudes, $K=4.55$ and $J=5.75 \mathrm{mag}$, give the same value of $\theta_{\mathrm{g}}$. These fitting parameters define the distance $d=1.0 \pm 0.15\left(R_{\mathrm{g}} / 86 R_{\odot}\right) \mathrm{kpc}$ and the luminosity $L_{\mathrm{g}}=850 \pm 250(d / 1 \mathrm{kpc})^{2} L_{\odot}$, where the radius $R_{\mathrm{g}}=86 \pm 13 R_{\odot}$ was determined by Schmutz et al. (1994) in the same way as for RW Hya.

Radiation from the ultraviolet. There is a striking similarity in the SEDs of SY Mus and RW Hya (Figs. 17 and 18), which suggests very similar ionization conditions in these systems. The evolution in the $\mathrm{H} \alpha$ line profile along the orbital motion is qualitatively of the same type in both systems (Schmutz et al. 1994; Schild et al. 1996). Our solution for the selected spectrum corresponds to $T_{\mathrm{h}}=110000 \mathrm{~K}$ ( $\equiv$ Zanstra temperature), $k_{\mathrm{h}}=4.8 \times 10^{-24}$ and $T_{\mathrm{e}}=18500 \pm 2500 \mathrm{~K}$ with scaling, $k_{\mathrm{N}}=2.5 \times 10^{15} \mathrm{~cm}^{-5}$, which gives the parameter $\delta=0.55$. These parameters can be discussed in the same way as those for RW Hya. Here the lower limit of the hot source temperature is $T_{\mathrm{h}}^{\min }=80000 \mathrm{~K}$. The only larger difference is in the relative contributions from the giant and those produced by the hot star in the $U$ band, which is due to the difference in $T_{\text {eff. }}$ This situation makes the amplitude $\Delta U$ (SY Mus) $>\Delta U$ (RW Hya) despite that orbital inclinations of both systems are comparable. As a by-product of fitting the UV spectra, we determined a new value of reddening to SY Mus, $E_{B-V}=0.35$. A larger quantity of $E_{B-V}=0.5-0.45$, previously suggested by MNSV and Pereira et al. (1995), yields too steep a far-UV continuum (from $\approx 1350 \AA$ ), which is not possible to match by any reasonable black-body radiation. The $2100-2300 \AA$ spectral region displays a higher continuum level than that at surrounding wavelengths if one consider the influence of the iron curtain. We note that this region is rich in strong absorption features (see in detail the well-exposed spectra, e.g. LWR12059).

\subsection{AR Pavonis}

Skopal et al. (2001b) recently summarized the historical, 1889-2001, LC of AR Pav. It is characterized by about $2 \mathrm{mag}$ deep minima - eclipses - and strong out-of-eclipse variations of between about 12 and $10 \mathrm{mag}$. This behaviour suggests that AR Pav persists in an active phase. The UV continuum shows the same type of profile during the whole IUE mission (e.g. Fig. 1 of Skopal 2003b). Therefore we selected observations well outside the giant's inferior conjunction (SWP13956 + LWR10570, 10/05/1981, $\varphi=0.37$ ) and just in the eclipse (SWP16949 + LWR13235, 13/05/1982, $\varphi=0.98$ ).

Radiation from the giant. We compared the RIJHKL fluxes with a synthetic spectrum of $T_{\text {eff }}=3400 \mathrm{~K}$ and $\theta_{\mathrm{g}}=(6.4 \pm$ $0.5) \times 10^{-10}$, which corresponds to the bolometric flux $F_{\mathrm{g}}^{\text {obs }}=$ $(3.1 \pm 0.3) \times 10^{-9} \mathrm{erg} \mathrm{cm}^{-2} \mathrm{~s}^{-1}$. The average reddening-free values of $J=7.96$ and $K=6.86$ mag yield $\theta_{\mathrm{g}}=6.5 \times 10^{-10}$
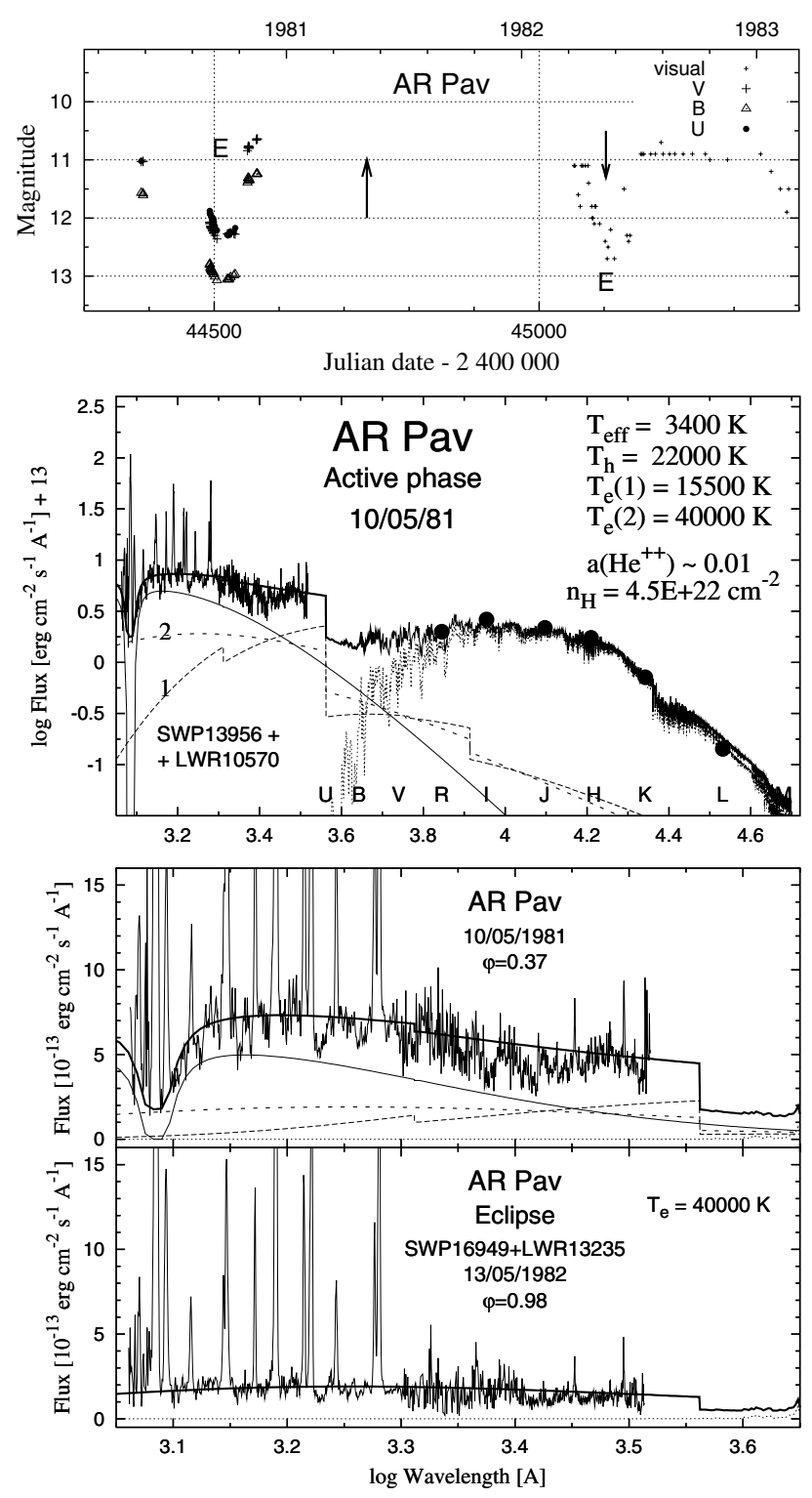

Fig. 19. The SED for AR Pav out and in the eclipse (positions of the spectra used are marked by arrows in the relevant part of the LC). Its out-of-eclipse profile in the ultraviolet resembles that observed in other eclipsing systems during active phases.

(Dumm \& Schild 1998). Schild et al. (2001) derived the distance $d=4.9 \mathrm{kpc}$ from the "rotational" giant's radius, $R_{\mathrm{g}}=$ $(130 \pm 25) R_{\odot}$ and the brightness surface relation for M-giants. This value is in particular agreement with that derived from a 106-day period of pulsations of the giant seen in some parts of the visual LC (Skopal et al. 2000). Our value of $\theta_{\mathrm{g}}$ then gives the radius of the giant $R_{\mathrm{g}}=(139 \pm 10) R_{\odot}$, which agrees with that determined by Schild et al. (2001). The uncertainty results only from that in $\theta_{\mathrm{g}}$. This quantity corresponds to the giant's luminosity, $L_{\mathrm{g}}=(2300 \pm 400)(d / 4.9 \mathrm{kpc})^{2} L_{\odot}$. According to the orbital solution, the separation between the components, $A \sin (i)=457 \pm 35 R_{\odot}$ (Quiroga et al. 2002) and the linear size of the giant's stellar disk that eclipses the object, $R_{\mathrm{g}}^{\mathrm{E}} / A=0.30 \pm 0.02$ (Skopal et al. 2000) limit the $R_{\mathrm{g}}$ radius to between $207 \pm 25 R_{\odot}\left(R_{\mathrm{g}} \sim R_{\mathrm{L}}, i=72^{\circ}\right)$ and $137 \pm 20 R_{\odot}$ $\left(i=90^{\circ}\right)$ (Eq. (25), see also Quiroga et al. 2002). From this 
point of view, $R_{\mathrm{g}}=(139 \pm 10) R_{\odot}$ corresponds to the orbital inclination $i=87 \pm 3^{\circ}$.

Radiation from the ultraviolet. The profile of the UV continuum on the out-of-eclipse spectra is of the same type as observed for other active symbiotics with a high orbital inclination: a Rayleigh attenuated far-UV continuum (here $n_{\mathrm{H}} \sim 4.5 \times$ $10^{22} \mathrm{~cm}^{-2}$ ), a rather cool radiation from the hot stellar source $\left(T_{\mathrm{h}} \sim 22000 \mathrm{~K}, k_{\mathrm{h}}=7.9 \times 10^{-23}\right)$, which is markedly affected by the iron curtain absorptions, and the nebular components of radiation from both the LTN $\left(T_{\mathrm{e}}=15500 \mathrm{~K}\right)$ and HTN $\left(T_{\mathrm{e}} \sim 40000 \mathrm{~K}\right)$. Radiation from the HTN is identified in all spectra. During eclipses, as the only light visible in the ultraviolet and on the out-of-eclipse spectra it is indicated by a non-zero level of the Rayleigh attenuated continuum near to the Ly $\alpha$ line. Its level is about $1-2 \times 10^{-13} \mathrm{erg} \mathrm{cm}^{-2} \mathrm{~s}^{-1} \AA^{-1}$, which corresponds to a large emission measure, $E M_{\mathrm{HTN}}=$ $2.9 \times 10^{60}(d / 4.9 \mathrm{kpc})^{2} \mathrm{~cm}^{-3}$ and/or the luminosity $L_{\mathrm{HTN}}=$ $530(d / 4.9 \mathrm{kpc})^{2} L_{\odot}$. Our solution is plotted in Fig. 19.

\subsection{AG Pegasi}

AG Peg is the slowest symbiotic nova. In the mid-1850 it rose in brightness from $\sim 9$ mag to $\sim 6$ mag and afterwards followed a gradual decline to the present brightness of $\sim 8.7 \mathrm{mag}$ in $V$. Evolution in its spectrum during the last century was described by many authors (e.g. Kenyon et al. 1993). The orbitally related wave-like variation in the LC developed around 1940 (Fig. 6 of Skopal 1998, and references therein). From that time to the present AGPeg faded from about 7.5 to 10 mag in the $B$ band. Therefore we selected two earlier observations within one orbital cycle, but at/around different conjunctions of the components (SWP03830 + LWR03376, 05/01/79, $\varphi=0.96$; SWP07407 + LWR06390, 14/12/79, $\varphi=0.38$ ) to show the orbitally-dependent nebular emission at a constant hot stellar radiation, and one observation taken later when the star's brightness significantly faded (Y1JO0308T + Y1JO0403T + Y1JO0309T + Y1JO0406T, 13/11/93, $\varphi=0.63)$. This was made by the Faint Object Spectrograph (FOS) on the board the Hubble Space Telescope.

Radiation from the giant. The cool component in AG Peg was classified as a normal M 3 III red giant (Mürset \& Schmid 1999; Kenyon et al. 1993). Its rather bright IR magnitudes suggest it cannot be very distant. The flux points corresponding to the RIJKLM measurements can be matched by a synthetic spectrum with $T_{\mathrm{eff}}=3600 \mathrm{~K}, \log (g)=1.0$ and $\theta_{\mathrm{g}}=2.4 \times$ $10^{-9}$, which yields the flux $F_{\mathrm{g}}^{\mathrm{obs}}=5.6 \times 10^{-8} \mathrm{erg} \mathrm{cm}^{-2} \mathrm{~s}^{-1}$. Our mean dereddened magnitudes, $K=3.85$ and $J=4.98 \mathrm{mag}$, also give a similar value of $\theta_{\mathrm{g}}=2.6 \times 10^{-9}$. Kenyon et al. (1993) determined the distance modulus for bolometric magnitudes under the assumption that the giant in AG Peg is similar to other red giants. They obtained $d=800 \mathrm{pc}$, which corresponds to $R_{\mathrm{g}}=d \times \theta_{\mathrm{g}}=85 R_{\odot}$ and its luminosity $L_{\mathrm{g}}=1100(d / 800 \mathrm{pc})^{2} L_{\odot}$. These quantities are identical to those determined by Kenyon et al. (1993). Other estimates in the literature also rely on common properties of red giants as a class. For example, MNSV employed the same method, but for $K$ magnitudes, and obtained $d=650 \mathrm{pc}$. As there is no
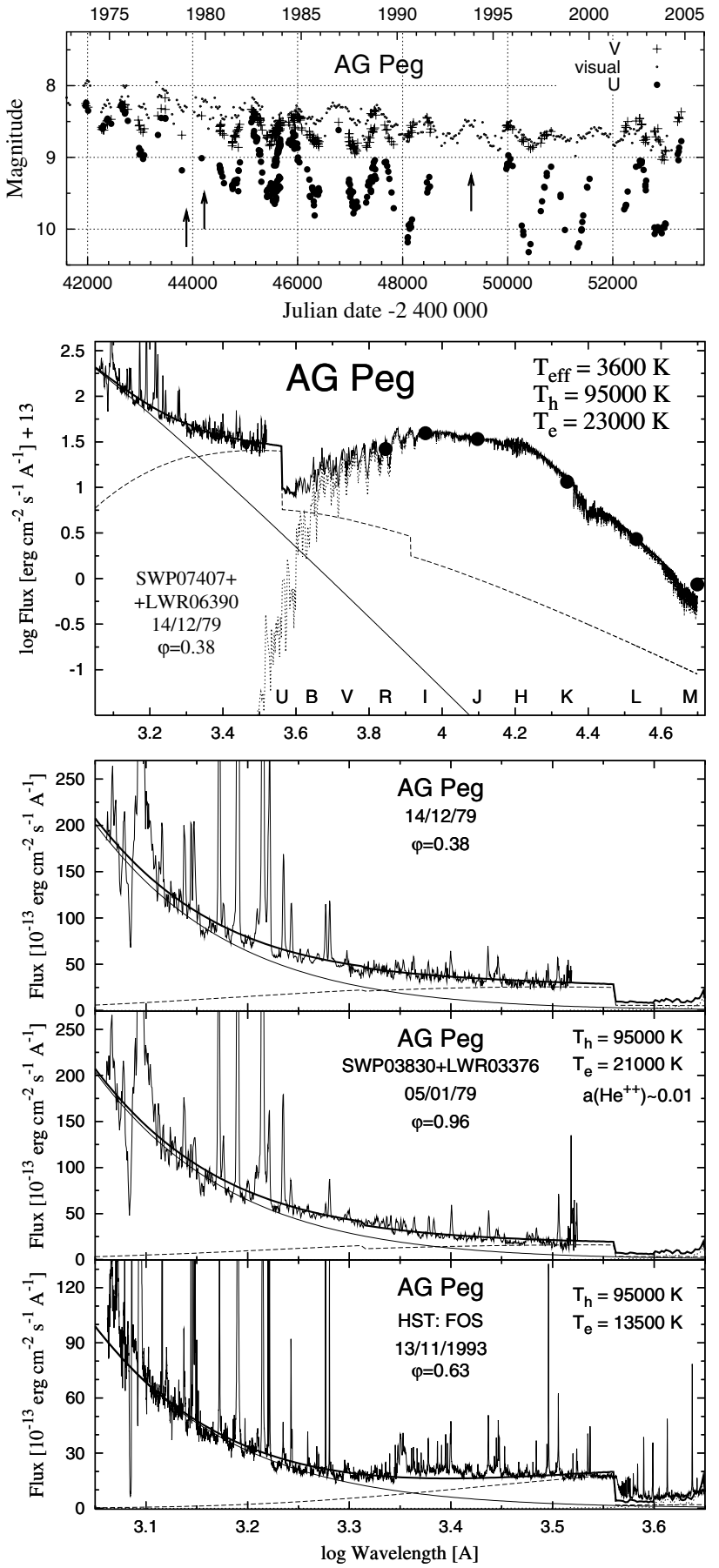

Fig. 20. The SED for AGPeg for three selected dates at different brightness of the system. Positions of the used spectra are marked by arrows in the LC (top). Data are from Luthardt (1984, 1989), Komárek (1990), Belyakina (1992), Tomov \& Tomova (1998, 2001), Skopal et al. (2004) and from 2003 our unpublished observations.

independent determination of the giant's radius in AG Peg we adopted that published by Kenyon et al. (1993), which conforms better to our solution.

Radiation from the ultraviolet. Properties of the UV spectrum and optical LCs during the investigated period resemble those of classical symbiotic stars during quiescent phases (Fig. 20). In addition, the overall fading of the star's brightness 
results from that in $L_{\mathrm{h}}$ (see Fig. 4 of Mürset \& Nussbaumer 1994, and Fig. 20 here). According to Mürset \& Nussbaumer (1994) a constant hot star temperature $T_{\mathrm{h}}=95000 \mathrm{~K}$ can be assumed during the period 1978-1993. We adopted this quantity in our modeling the SED. The following points are relevant.

(i) The 1979 spectra show the phase-dependence of the nebular radiation, which follows that in the LCs. The observed $E M$ increased by a factor of about 2 from $05 / 01 / 79$ $(\varphi=0.96)$ to $14 / 12 / 79(\varphi=0.38)$. In contrast to, e.g. RW Hya and SY Mus, the far-UV continuum is not subject to orbitally-related variation, which suggests that the AG Peg orbit is not highly inclined to the observer.

(ii) We estimated the lower limit of the hot star temperature, $T_{\mathrm{h}}^{\min }=70000 \mathrm{~K}$ for the $14 / 12 / 79$ spectrum. The $\mathrm{HII}$ region in AGPeg is very open (Fig. 11 of Kenyon et al. 1993), which means that a significant fraction of the $L_{\mathrm{ph}}$ photons escapes the system. This implies $T_{\mathrm{h}} \gg T_{\mathrm{h}}^{\min }$ to produce the observed $E M$ for an appropriate fit. For example, if only one half of the ionizing photons gives rise to the observed nebular emission then the hot star temperature has to increase to $T_{\mathrm{h}}=100000 \mathrm{~K}$ to fit the far-UV continuum (Eq. (23) for $\delta=0.5$ ).

(iii) The situation for AGPeg is complicated by its hot star wind $\left(v_{\infty} \sim 900-1000 \mathrm{~km} \mathrm{~s}^{-1}, \dot{M} \sim 3 \times 10^{-7} M_{\odot} \mathrm{yr}^{-1}\right.$, Vogel \& Nussbaumer 1994), which represents an additional source of emitters contributing to the observed nebula. However, the emission measure from the hot star wind, $E M_{\mathrm{W}}$, is negligible with respect to that observed. We estimated its quantity to be $E M_{\mathrm{W}} \doteq 8.3 \times 10^{58} \mathrm{~cm}^{-3}$ by using relation (8) of Skopal et al. (2002a) for $R_{\star} \equiv$ $R_{\mathrm{h}}^{\text {eff }}=0.18 R_{\odot}$, the radius $r_{\text {min }} \sim 1.5 R_{\star}$, from which the wind becomes optically thin (Leitherer 1988) and the parameter $\beta=1$ as assumed for hot star winds. Thus $E M_{\mathrm{W}} \ll E M^{\text {obs }}$. This suggests that a major part of the nebular emission has to come from a more dense region in the system, which could be associated with the colliding region of the two winds located probably at the vicinity of the giant's hemisphere facing the hot star (Kenyon et al. 1993).

(iv) Finally, the 1993-spectrum shows a higher efficiency in producing the nebular component of radiation $(\delta=0.73)$. A decrease of the mass-loss rate from the hot star by a factor of $\sim 5$ (Vogel \& Nussbaumer 1994) results in a larger expansion of the massive giant's wind towards the hot star and thus in a larger opening of the colliding zone. This makes it possible for a larger fraction of the $L_{\text {ph }}$ photons to be involved in the ionization/recombination process. A quantitative model should support this view.

\subsection{AX Persei}

The eclipsing nature of this classical symbiotic star was unambiguously revealed by photometric observations of the $\sim 1$ mag deep narrow minimum - eclipse - in the UBV LCs during its recent 1989 major outburst (Skopal 1991). The historical LC of AXPer is characterized by long-lasting periods of quiescence with superposition of a few bright
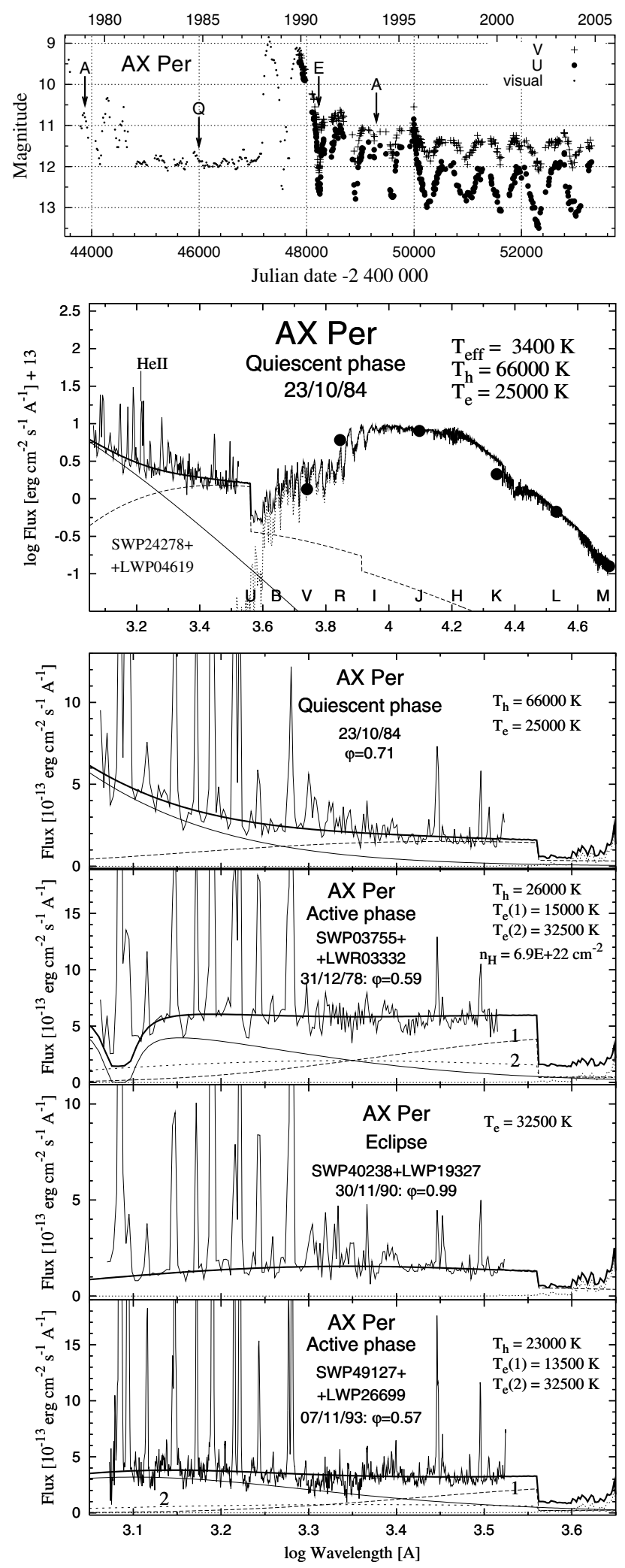

Fig. 21. The SED for AXPer during the activity, quiescence and eclipse. Positions of the spectra used are marked by arrows in the LC. Small dots represent means of the CDS visual data in the 30-day bins.

stages (Skopal et al. 2001a). Accordingly we selected four IUE spectra taken at very different brightness/activity levels: during quiescence (SWP24278 + LWP04619, 23/10/84, 
$\varphi=0.71$ ), during the 1978 outburst (SWP03755 + LWR03332, $31 / 12 / 78, \varphi=0.59$ ), during the 1990 eclipse (SWP40238+ LWP19327, 30/11/90, $\varphi=0.99)$ and during the transition to quiescence (SWP49127 + LWP26699, 07/11/93, $\varphi=0.57$ ).

Radiation from the giant. Infrared photometry of AX Per was summarized by Skopal (2000), who derived the following parameters of the giant in the same way as in this paper: $T_{\mathrm{eff}}=3400 \pm 150 \mathrm{~K}, \theta_{\mathrm{g}}=(1.3 \pm 0.2) \times 10^{-9}$, $F_{\mathrm{g}}^{\mathrm{obs}}=(1.4 \pm 0.3) \times 10^{-8} \mathrm{erg} \mathrm{cm}^{-2} \mathrm{~s}^{-1}$. The angular radius then gives the distance $d=1700 \pm 200\left(R_{\mathrm{g}} / 102 R_{\odot}\right) \mathrm{pc}$, where the giant's radius was derived from the eclipse timing under the assumption that $i=90^{\circ}$, which is supported by a rectangular profile of the minimum (Skopal 1994). The giant's corresponding luminosity $L_{\mathrm{g}}=1200 \pm 400(d / 1.7 \mathrm{kpc})^{2} L_{\odot}$.

Radiation from the ultraviolet: quiescent phase. The best solution of the 23/10/84 spectrum yields a low temperature of the observed hot stellar source, $T_{\mathrm{h}}=66000+15000 /-7000 \mathrm{~K}$ $\left(k_{\mathrm{h}}=1.7 \times 10^{-24}\right)$, but a strong nebular emission characterized by $T_{\mathrm{e}}=25000 \pm 3000 \mathrm{~K}$ and scaled with $k_{\mathrm{N}}=7.2 \times$ $10^{14} \mathrm{~cm}^{-5}$. These parameters give $\delta=2.3$ (Eq. (21)), i.e. the corresponding amount of the $L_{\mathrm{ph}}$ photons is not capable of producing the observed quantity of the nebular emission. This situation signals the presence of a material around the accretor of a disk-like structure, which makes it optically thick to a larger distance from the hot surface of the central star $\left(\geq R_{\mathrm{h}}^{\text {eff }}\right)$ in directions on the orbital plane (i.e. on the lines of sight; $i \sim 90^{\circ}$ ). This means that in AX Per the accreted matter has a disk-like structure even during quiescent phases. For comparison, we also present a formal solution for $\delta=1$, i.e. $T_{\mathrm{h}}=T_{\mathrm{h}}^{\min } \doteq 100000 \mathrm{~K}$ (Table 3 ). The solution for this case gives $R_{\mathrm{h}}^{\text {eff }}=0.065(d / 1.7 \mathrm{kpc}) R_{\odot}$ and $L_{\mathrm{h}}=380(d / 1.7 \mathrm{kpc})^{2} L_{\odot}$, which are similar to those determined by MNSV. Our somewhat larger value of $L_{\mathrm{h}}$ is probably caused by different scaling, because we considered the influence of the iron curtain absorptions.

Radiation from the ultraviolet: active phases. It is characterized by a flat profile with signatures of the Rayleigh attenuated far-UV continuum and the influence of the iron curtain absorptions. An appropriate discussion of such a spectrum was introduced, for example, in Sect. 4.10 for CICyg. Also here the three-component model, composed of the HSS, LTN and HTN radiative contributions, has to be applied to get a satisfactory fit to observations. Fitting and derived parameters of our solutions for both selected spectra $(31 / 12 / 78,07 / 11 / 93)$ are given in Table 4 and Fig. 21.

Radiation from the ultraviolet: eclipse. The UV spectrum during the 1990-eclipse can be matched by the HTN radiating at $T_{\mathrm{e}}=32500 \pm 3000 \mathrm{~K}$ and scaled with $k_{\mathrm{N}}=$ $8.0 \times 10^{14} \mathrm{~cm}^{-5}$. The same component was used in fitting the $31 / 12 / 78$ spectrum to fill in the Rayleigh attenuated far-UV continuum. However, during the 1994-eclipse (SWP52027 + LWP29094, 04/09/94, $\varphi=0.01$ ), at transition to quiescence, the level of the HTN emission was a factor of about 2.5 fainter. This suggests that the amount of the HTN contribution is a function of the activity.

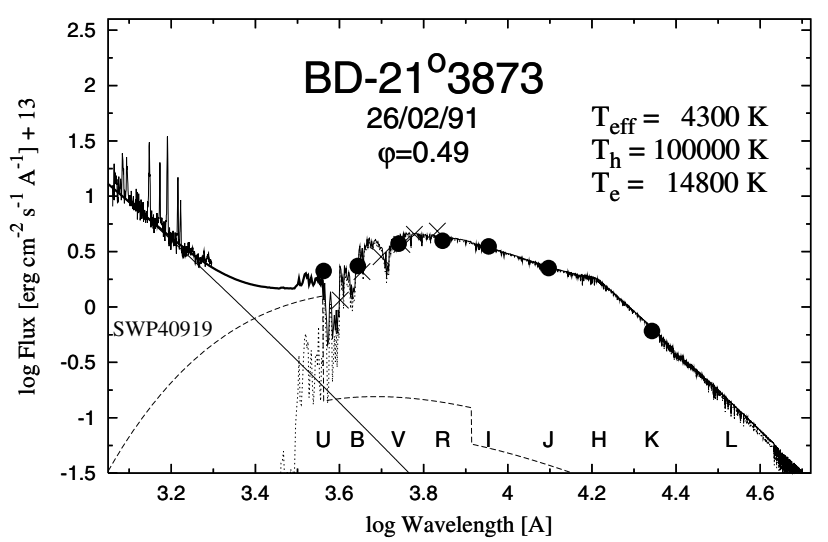

Fig. 22. Approximate SED for BD - $21^{\circ} 3873$ given by its SWP spectrum in the ultraviolet and UBVRIJK fluxes from photometric measurements $(\bullet)$. The value of $U(\varphi=0.31)$ from Munari et al. (1992) was extrapolated to $U(\varphi=0.5)$ according to the profile of the $u$-LC. Crosses $(\times)$ represents flux-points from the spectrum taken on $24 / 02 / 88$ ( $\varphi=0.61)$ by Niehues et al. (1992).

\subsection{2. $B D-21^{\circ} 3873$}

Smith et al. (1997) presented the largest set of $u v b y$ photometry of this symbiotic star. The LCs show a pronounced orbitallyrelated variation with superposed double-sine patterns in $y, b$ and $v$. There are no signatures of an active phase (also Fig. 9 of Skopal et al. 2002b). As for LT Del only SWP spectrum was available in the IUE archive (SWP40919, 26/02/91, $\varphi=0.49$ ). We determined a new value of $E_{B-V}=0.20$ by the same way as for LT Del (Sect. 4.12).

Radiation from the giant. Fluxes of the BVRIJK photometric measurements can be compared well with a synthetic spectrum calculated for $T_{\text {eff }}=4300 \mathrm{~K}$ with agreement with the atmospheric analysis of Pereira \& Mello (1997) and Smith et al. (1997). The scaling, $k_{\mathrm{g}}=2.4 \times 10^{-19}$, then gives the bolometric flux $F_{\mathrm{g}}^{\mathrm{obs}}=4.7 \times 10^{-9} \mathrm{erg} \mathrm{cm}^{-2} \mathrm{~s}^{-1}$. From the surface gravity, $\log (g)=1.69 \pm 0.17$ (Pereira \& Mello 1997), one can derive the giant's radius as $R_{\mathrm{g}}=(29 \pm 6)\left(M_{\mathrm{g}} / 1.5 M_{\odot}\right)^{1 / 2} R_{\odot}$ and the distance $d=R_{\mathrm{g}} / \theta_{\mathrm{g}}=(1.3 \pm 0.3)\left(M_{\mathrm{g}} / 1.5 M_{\odot}\right)^{1 / 2} \mathrm{kpc}$, where the giant's mass was adopted as for AGDra, because of their very similar atmospheric parameters (Smith et al. 1997). The giant's luminosity $L_{\mathrm{g}}=260(d / 1.3 \mathrm{kpc})^{2} L_{\odot}$.

Radiation from the ultraviolet. Observed and approximate modeled SEDs are shown in Fig. 22. As for LT Del we adopted $T_{\mathrm{h}} \equiv 100000 \mathrm{~K}$, which requires a scaling of $k_{\mathrm{h}}=1.7 \times$ $10^{-24}$ and suggests parameters for the nebular emission, $T_{\mathrm{e}} \sim$ $15000 \mathrm{~K}$ and $k_{\mathrm{N}} \sim 3.3 \times 10^{14} \mathrm{~cm}^{-5}$. This solution gives $\delta=0.36$. Also $T_{\mathrm{h}} \equiv 75000 \mathrm{~K}$ (Schmid \& Nussbaumer 1993) is above $T_{\mathrm{h}}^{\min }$, because the corresponding $\delta=0.63$. This results suggest an open ionized zone $(X>1)$, which is important in calculating the mass-loss rate from the giant (Sect. 5.2.1).

\section{Summary}

By disentangling the composite spectrum of 21 S-type symbiotic stars we isolated four basic components of radiation contributing to their UV/optical/IR continuum: Radiation from the (i) cool giant; (ii) hot stellar source (HSS); 
(iii) low-temperature nebula (LTN) and (iv) high-temperature nebula (HTN). Physical parameters of these components are introduced in Tables 2-4 and corresponding solutions are shown in the relevant figures. During quiescent phases, components from the hot object show orbitally-related variations and unpredictable changes in $T_{\mathrm{e}}, L_{\mathrm{h}}$ and $E M$. Transition to active phases is followed by a dramatic change in $T_{\mathrm{h}}$ and by the appearance of the HTN component in the spectrum. Below we summarize and discuss their common properties and observed effects.

\subsection{Radiation from the giant}

This component of radiation in the spectra of S-type symbiotic stars is determined by two fitting parameters - the angular radius, $\theta_{\mathrm{g}}$ and the effective temperature, $T_{\text {eff }}$ (Sect. 3.1). Both are connected throughout the observed bolometric flux, $F_{\mathrm{g}}^{\mathrm{obs}}=\theta_{\mathrm{g}}^{2} \sigma T_{\mathrm{eff}}^{4}$, which defines the giant's luminosity as

$L_{\mathrm{g}}=4 \pi d^{2} F_{\mathrm{g}}^{\mathrm{obs}}$.

To test common properties of giants we examined the quantity of $F_{\mathrm{g}}^{\mathrm{obs}}$ as a function of the distance $d$ in the sense of the last relation. Fitting the relevant quantities from Tables 1 and 2 determines this function as

$\log \left(F_{\mathrm{g}}^{\mathrm{obs}}\right)=-2 \log (d)-(7.30 \pm 0.05)$ for red giants

and

$\log \left(F_{\mathrm{g}}^{\mathrm{obs}}\right)=-2 \log (d)-(8.03 \pm 0.05)$ for yellow giants.

The flux is in erg $\mathrm{cm}^{-2} \mathrm{~s}^{-1}$ and the distance in kpc. The constant corresponds to a characteristic luminosity $L_{\mathrm{g}}=1600 \pm$ 200 and $290 \pm 30 L_{\odot}$ for red and yellow giants, respectively. Figure 23 plots the observed data and fits. In the figure we distinguish giants having independently determined radii (from eclipses, co-rotation, atmospheric analysis, lobe-filling giant; 11 objects), which then provide the distance from their $\theta_{\mathrm{g}}$. For CQ Dra and CH Cyg we adopted their HIPPARCOS distances. This group of giants confirms that cool components in S-type systems are normal giants. Otherwise the distances were estimated from $S T / R_{\mathrm{g}}$ dependencies (Belle et al. 1999) and the measured angular radii. $\mathrm{CHCyg}$, which is suspected to contain two red giants in accordance with its triple-star model, was not included in the analysis. Finally, having the SED in the infrared, these relations can be used to estimate distances of S-type symbiotic stars.

\subsection{The quiescent phase}

\subsubsection{Nebular continuum radiation}

General properties: our solutions for 15 symbiotic stars during the quiescent phase showed that the nebular component of radiation can be characterized by a unique electron temperature. For individual objects $T_{\mathrm{e}}$ runs between about 12000 and $25000 \mathrm{~K}$ with an average value of $19000 \mathrm{~K}$ (Table 3). For $\mathrm{Z}$ And and AG Peg we observed a large decrease in $T_{\mathrm{e}}$ by about $10^{4} \mathrm{~K}$. In the case of $\mathrm{Z}$ And, $T_{\mathrm{e}}$ decreased at approximately the same $L_{\mathrm{N}}$ and probably also $L_{\mathrm{h}}$, while for AG Peg this change

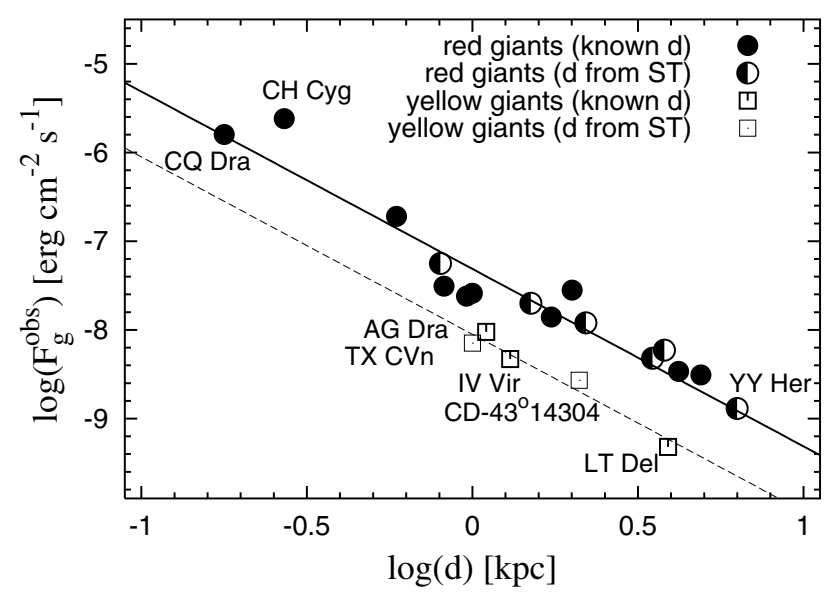

Fig. 23. The relationship between the observed bolometric flux of giants, $F_{\mathrm{g}}^{\mathrm{obs}}$ and the distance $d$. Giants with independently known radii or distances are distinguished (see the text). Data are from Tables 1 and 2. Solid and dashed line correspond to their fit given by relations (27) and (28). CH Cyg was omitted in the fitting (see the text).

Table 2. Fitting and derived parameters: cool components.

\begin{tabular}{cccrr}
\hline \hline Object & $\begin{array}{c}T_{\text {eff }} \\
{[\mathrm{K}]}\end{array}$ & $\theta_{\mathrm{g}}$ & $\begin{array}{c}R_{\mathrm{g}} \\
{\left[R_{\odot}\right]}\end{array}$ & $\begin{array}{c}L_{\mathrm{g}} \\
{\left[L_{\odot}\right]}\end{array}$ \\
\hline EG And & 3500 & $4.7 \times 10^{-9}$ & 124 & 2070 \\
Z And & 3400 & $1.6 \times 10^{-9}$ & 106 & 1400 \\
AE Ara & 3200 & $9.0 \times 10^{-10}$ & 140 & 1800 \\
CD-43 14304 & 4000 & $4.3 \times 10^{-10}$ & 40 & 320 \\
TX CVn & 4100 & $6.7 \times 10^{-10}$ & 30 & 230 \\
T CrB & 3400 & $1.8 \times 10^{-9}$ & 75 & 680 \\
BF Cyg & 3400 & $8.8 \times 10^{-10}$ & 150 & 2700 \\
CHCyg & 2600 & $3.1 \times 10^{-8}$ & 370 & 5600 \\
CICyg & 3300 & $2.0 \times 10^{-9}$ & 180 & 3400 \\
V1329 Cyg & 3300 & $7.2 \times 10^{-10}$ & 132 & 1900 \\
LT Del & 4100 & $1.7 \times 10^{-10}$ & 30 & 230 \\
AG Dra & 4300 & $7.0 \times 10^{-10}$ & 33 & 360 \\
CQ Dra & 3700 & $1.2 \times 10^{-8}$ & 96 & 1600 \\
V443 Her & 3300 & $1.3 \times 10^{-9}$ & 130 & 1800 \\
YY Her & 3500 & $3.9 \times 10^{-10}$ & 110 & 1600 \\
RW Hya & 3800 & $1.6 \times 10^{-9}$ & 59 & 650 \\
SY Mus & 3400 & $1.9 \times 10^{-9}$ & 86 & 850 \\
AR Pav & 3400 & $6.4 \times 10^{-10}$ & 139 & 2300 \\
AG Peg & 3600 & $2.4 \times 10^{-9}$ & 85 & 1100 \\
AX Per & 3400 & $1.3 \times 10^{-9}$ & 102 & 1200 \\
BD - 21 ${ }^{\circ} 3873$ & 4300 & $4.9 \times 10^{-10}$ & 29 & 260 \\
\hline
\end{tabular}

Useful conversions according to the Sect. 3.1: $k_{\mathrm{g}}=\theta_{\mathrm{g}}^{2}=F_{\mathrm{g}}^{\mathrm{obs}} / \sigma T_{\mathrm{eff}}^{4}$, $R_{\mathrm{g}}=d \times \theta_{\mathrm{g}}, \quad L_{\mathrm{g}}=4 \pi d^{2} F_{\mathrm{g}}^{\mathrm{obs}}$ and $d$ in Table 1.

was followed by a significant decrease in $L_{\mathrm{N}}$, obviously caused by that in $L_{\mathrm{h}}$ and thus $L_{\mathrm{ph}}$ (Table 3 ). A decrease in $T_{\mathrm{e}}$ could be caused by a decrease of the hot star wind, which causes removal of a fraction of hot electrons from the plasma created by collisions. For EG And and CQ Dra a transient enhancement of the emission measure observed at the same orbital phase can result from a relevant increase in the mass-loss rate from the giant, which thus supplies more emitters into the ionized zone. This 
Table 3. Fitting and derived parameters: hot components during quiescent phases.

\begin{tabular}{|c|c|c|c|c|c|c|c|c|c|c|c|}
\hline "Object & Date & Phase & $\begin{array}{r}T_{\mathrm{h}} \\
{[\mathrm{K}]}\end{array}$ & $\overline{\theta_{\mathrm{h}}}$ & $\begin{array}{l}R_{\mathrm{h}}^{\text {eff }} \\
{\left[R_{\odot}\right]}\end{array}$ & $\begin{array}{c}L_{\mathrm{h}} \\
{\left[L_{\odot}\right]}\end{array}$ & $\begin{array}{c}T_{\mathrm{e}} \\
{[\mathrm{K}]}\end{array}$ & $\begin{array}{c}E M \\
{\left[\mathrm{~cm}^{-3}\right]}\end{array}$ & $\begin{array}{l}L_{\mathrm{N}} \\
L_{\odot}\end{array}$ & $\overline{\bar{C}}$ & $\begin{array}{c}\log \left(\dot{M}_{\mathrm{W}}\right) \\
{\left[M_{\odot} \mathrm{yr}^{-1}\right]}\end{array}$ \\
\hline \multirow[t]{3}{*}{ EG And } & $10 / 09 / 95$ & 0.52 & 95000 & $1.2 \times 10^{-12}$ & 0.032 & 77 & 25000 & $5.2 \times 10^{58}$ & 12 & 1.0 & $-6.46^{a}$ \\
\hline & $28 / 08 / 91$ & 0.48 & $"$ & $1.1 \times 10^{-12}$ & 0.029 & 60 & 13500 & $1.0 \times 10^{58}$ & 2.6 & 0.46 & $-6.80^{b}$ \\
\hline & $13 / 01 / 83$ & 0.95 & $"$ & $"$ & $"$ & " & 14500 & $7.5 \times 10^{57}$ & 1.9 & 0.33 & \\
\hline \multirow[t]{3}{*}{ Z And } & $19 / 11 / 82$ & 0.49 & $>120000$ & $<1.7 \times 10^{-12}$ & $<0.11$ & $>2300$ & 20500 & $9.8 \times 10^{59}$ & 220 & $<0.92$ & -6.10 \\
\hline & $03 / 02 / 88$ & 0.99 & $"$ & $<1.0 \times 10^{-12}$ & $<0.068$ & $>860$ & 22000 & $1.6 \times 10^{59}$ & 35 & $<0.20$ & \\
\hline & $29 / 06 / 95$ & 0.57 & $"$ & $<1.7 \times 10^{-12}$ & $<0.11$ & $>2300$ & 11500 & $7.3 \times 10^{59}$ & 200 & $<1.1$ & -6.16 \\
\hline \multirow[t]{3}{*}{ BF Cyg } & $10 / 04 / 92$ & 0.46 & 95000 & $3.0 \times 10^{-12}$ & 0.51 & 18800 & 18000 & $6.2 \times 10^{60}$ & 1400 & 0.72 & -6.20 \\
\hline & & & & & & & 42000 & $5.7 \times 10^{60}$ & 1050 & 0.31 & \\
\hline & $26 / 09 / 96$ & 0.61 & 95000 & $2.9 \times 10^{-12}$ & 0.50 & 18100 & 22800 & $3.1 \times 10^{60}$ & 670 & 0.24 & -6.34 \\
\hline CICyg & $22 / 05 / 89$ & 0.82 & 115000 & $1.2 \times 10^{-12}$ & 0.11 & 1700 & 24000 & $4.8 \times 10^{59}$ & 110 & 0.48 & -6.88 \\
\hline \multirow[t]{2}{*}{ V1329 Cyg } & $29 / 03 / 83$ & 0.57 & $>170000$ & $<5.4 \times 10^{-13}$ & $<0.10$ & $>7500$ & 18000 & $2.3 \times 10^{60}$ & 530 & $<0.95$ & -5.87 \\
\hline & $07 / 08 / 81$ & 0.9 & $"$ & $3.6 \times 10^{-13}$ & 0.068 & 3400 & 18000 & $4.8 \times 10^{59}$ & 110 & 0.43 & \\
\hline LT Del & $16 / 06 / 90$ & 0.49 & $100000^{g}$ & $6.7 \times 10^{-13}$ & 0.12 & 1200 & 17000 & $1.5 \times 10^{59}$ & 35 & 0.28 & -6.8 \\
\hline \multirow[t]{2}{*}{ AG Dra } & $27 / 10 / 89$ & 0.62 & $>110000$ & $<7.3 \times 10^{-13}$ & $<0.034$ & $>153$ & 21500 & $8.0 \times 10^{58}$ & 18 & $<0.93$ & -6.49 \\
\hline & $25 / 09 / 79$ & 0.91 & " & $"$ & $"$ & $"$ & 21800 & $5.8 \times 10^{58}$ & 13 & 0.68 & \\
\hline \multirow[t]{2}{*}{ CQ Dra } & $23 / 10 / 84$ & 0.80 & 110000 & $8.9 \times 10^{-13}$ & 0.007 & 6.6 & 19500 & $2.6 \times 10^{57}$ & 0.63 & 0.84 & $-6.92^{c}$ \\
\hline & $11 / 10 / 86$ & 0.22 & $>145000$ & $<7.5 \times 10^{-13}$ & $<0.006$ & $>14$ & 22000 & $5.8 \times 10^{57}$ & 1.4 & $<0.94$ & $-6.60^{d}$ \\
\hline \multirow[t]{3}{*}{ V443 Her } & $20 / 10 / 80$ & 0.47 & $>39000^{\dagger}$ & $<4.8 \times 10^{-12}$ & $<0.47$ & $>460$ & 15500 & $5.8 \times 10^{59}$ & 140 & $<3.9$ & \\
\hline & & & $75000^{\ddagger}$ & $2.2 \times 10^{-12}$ & 0.21 & 1300 & " & $"$ & $"$ & 1.0 & -6.42 \\
\hline & $21 / 10 / 91$ & 0.23 & $>39000^{\dagger}$ & $<4.8 \times 10^{-12}$ & $<0.47$ & $>460$ & 12000 & $2.3 \times 10^{59}$ & 63 & $<2.0$ & \\
\hline YY Her & $12 / 08 / 80$ & 0.46 & $>105000$ & $<4.9 \times 10^{-13}$ & $<0.14$ & $>2100$ & 21500 & $1.1 \times 10^{60}$ & 250 & $<1.0$ & -6.17 \\
\hline \multirow[t]{2}{*}{ RW Hya } & $19 / 07 / 79$ & 0.32 & 110000 & $2.5 \times 10^{-12}$ & 0.091 & 1100 & 15000 & $2.3 \times 10^{59}$ & 57 & 0.54 & -6.50 \\
\hline & $24 / 05 / 87$ & 0.06 & & & & & 40000 & $6.0 \times 10^{58}$ & 11 & & \\
\hline SYMus & $29 / 11 / 81$ & 0.62 & 110000 & $2.2 \times 10^{-12}$ & 0.097 & 1200 & 18500 & $3.0 \times 10^{59}$ & 69 & 0.55 & -6.39 \\
\hline \multirow[t]{3}{*}{ AG Peg } & $14 / 12 / 79$ & 0.38 & 95000 & $5.2 \times 10^{-12}$ & 0.18 & 2400 & 23000 & $8.3 \times 10^{59}$ & 173 & 0.58 & -6.19 \\
\hline & $05 / 01 / 79$ & 0.96 & 95000 & $5.2 \times 10^{-12}$ & 0.18 & 2400 & 21000 & $4.4 \times 10^{59}$ & 94 & 0.33 & \\
\hline & $13 / 11 / 93$ & 0.63 & 95000 & $3.6 \times 10^{-12}$ & 0.13 & 1180 & 13500 & $3.2 \times 10^{59}$ & 79 & 0.73 & -6.38 \\
\hline \multirow[t]{3}{*}{ AXPer } & $23 / 10 / 84$ & 0.71 & $>66000^{\dagger}$ & $<1.3 \times 10^{-12}$ & $<0.098$ & $>170$ & 25000 & $2.5 \times 10^{59}$ & 53 & $<2.3$ & \\
\hline & & & $100000^{\ddagger}$ & $8.7 \times 10^{-13}$ & 0.065 & 400 & $"$ & $"$ & $"$ & 1.0 & $-6.14^{e}$ \\
\hline & & & 140000 & $6.7 \times 10^{-13}$ & 0.051 & 900 & $"$ & $"$ & $"$ & 0.54 & $-6.26^{h}$ \\
\hline IV Vir $^{f}$ & $26 / 02 / 91$ & 0.49 & $100000^{g}$ & $1.3 \times 10^{-12}$ & $<0.072$ & 480 & 14800 & $6.7 \times 10^{58}$ & 16 & 0.36 & -7.05 \\
\hline
\end{tabular}

${ }^{\dagger} T_{\mathrm{h}}$ given by the fit; ${ }^{\ddagger} T_{\mathrm{h}}=T_{\mathrm{h}}^{\mathrm{min}} ;{ }^{a} X=0.73 ;{ }^{b} X=0.65 ;{ }^{c} X=1.5 ;{ }^{d} X=0.51 ;{ }^{e} X=1.0 ;{ }^{h} X=20 ;{ }^{f}=\mathrm{BD}-21{ }^{\circ} 3873 ;{ }^{g}$ adopted value.

change has a counterpart in the increase of the star's brightness in $U$.

Mass-loss rate from the giant: the nebula during quiescence arises from ionization of the cool giant wind. Skopal (2001a) showed that the observed emission measure is consistent with that produced by the ionization model. Therefore we can calculate $E M$ directly by integrating emission contributions throughout the volume of the fully ionized zone as

$E M=\int_{\mathrm{H} \text { II }} n^{2}(r) \mathrm{d} V$,

which can be determined through parameters of the giant's wind. The particle density $n(r)$ satisfies the equation of continuity, $\dot{M}_{\mathrm{W}}=4 \pi r^{2} \mu m_{\mathrm{H}} n(r) v_{\mathrm{W}}(r)$, in which the velocity structure of the wind, $v_{\mathrm{W}}(r)=v_{\infty}\left(1-R_{\mathrm{g}} / r\right)^{\beta}$ (Castor et al. 1975), $v_{\infty}$ is the terminal velocity of the wind and the parameter $\beta$ characterizes its acceleration; $\beta=2.5$ satisfies a wind from a late-type giant (Schröder 1985). Under these conditions the upper limit of the $E M$ calculated in accordance with the relation (29) can be expressed analytically as

$E M=\frac{-1}{16 \pi\left(\mu m_{\mathrm{H}}\right)^{2}}\left(\frac{\dot{M}_{\mathrm{W}}}{v_{\infty}}\right)^{2} \frac{1}{R_{\mathrm{g}}}\left[1-\left(1-\frac{R_{\mathrm{g}}}{Q}\right)^{-4}\right]$

(Nussbaumer \& Vogel 1989; Skopal 2001a), which is applicable for extended ionized zones in symbiotic binaries (i.e. very open in the sense of the STB model; parameter $X \gg 1)$. This solution corresponds to an upper limit of the $E M$ assuming the sphere around the cool star to be fully ionized from $r=Q$ to $r=\infty$. The parameter $Q$ is the location of the $\mathrm{HI} / \mathrm{H}$ II boundary on the binary axis and the wind starts at the giant's surface. We comment on our calculations of $\dot{M}_{\mathrm{W}}$ as follows:

(i) the mass-loss rate estimated from relation (30) represents its lower limit. In the real case the ionized volume is smaller. It represents only a fraction of the whole sphere, which requires a higher $\dot{M}_{\mathrm{W}}$ to give rise the 
Table 4. Fitting and derived parameters: hot components during active phases.

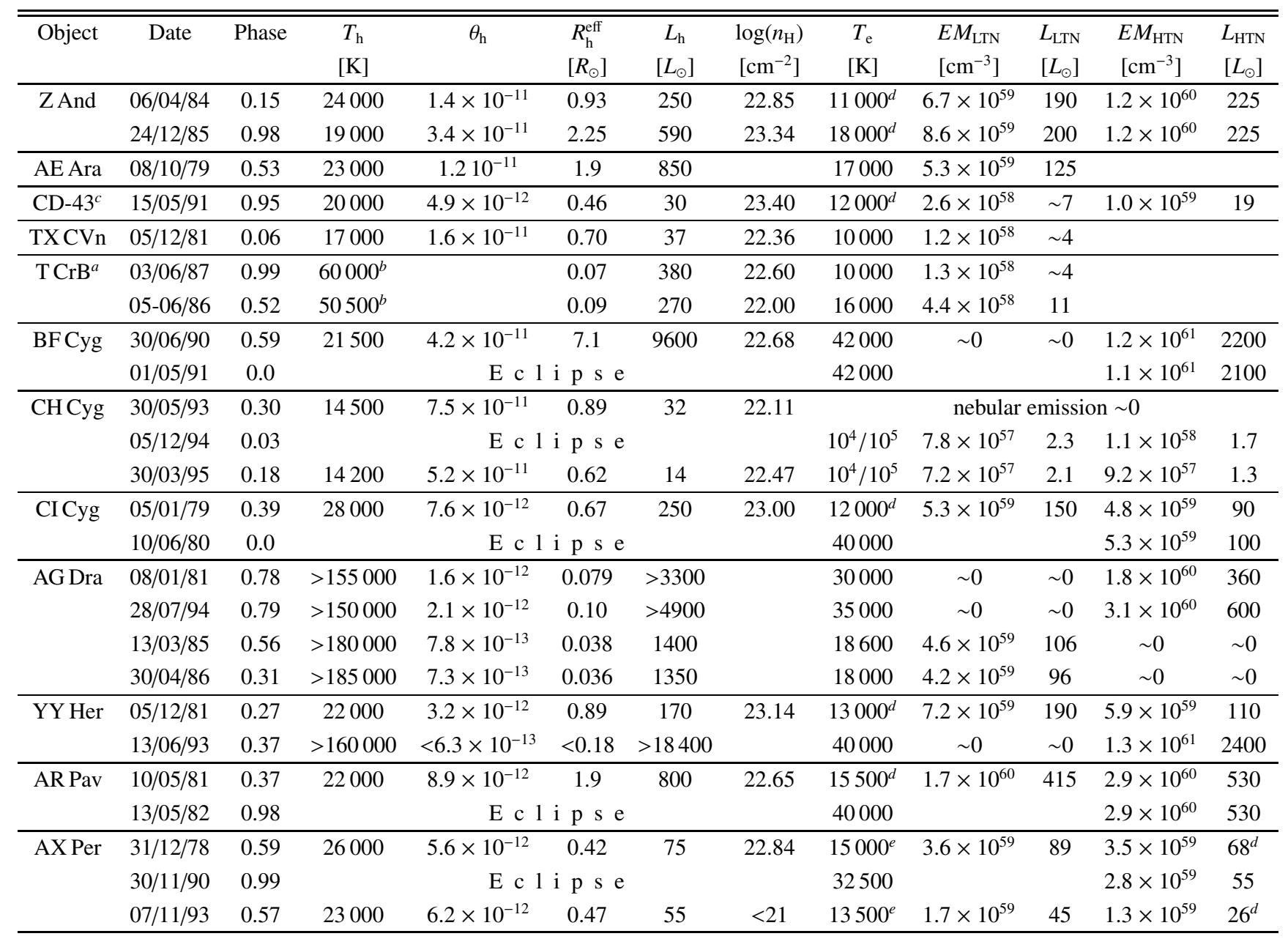

${ }^{a}$ accretion disk model, see Sect. $4.7 ;{ }^{b} T_{\max }$ of the AD-model; ${ }^{c} \mathrm{CD}-43^{\circ} 14304 ;{ }^{d} T_{\mathrm{e}}(\mathrm{HTN})=40000 \mathrm{~K} ;{ }^{e} T_{\mathrm{e}}(\mathrm{HTN})=32500 \mathrm{~K}$.

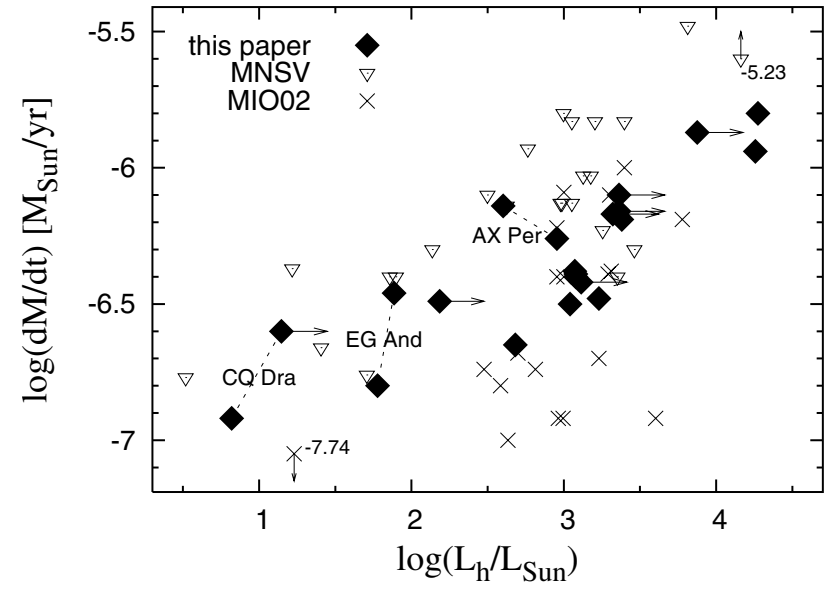

Fig. 24. Comparison of our mass-loss rates from Table 3 with those published by MNSV ( $\nabla$, recalculated to our $d$ and $v_{\infty}=20 \mathrm{~km} \mathrm{~s}^{-1}$ ) and by Mikolajewska et al. (2002b) ( $\times$, obtained from radio observations and scaled with $v_{\infty}=20 \mathrm{~km} \mathrm{~s}^{-1}$ ). Horizontal arrows indicate a shift in the luminosity by a factor of 2 for values of $T_{\mathrm{h}}>T_{\mathrm{h}}^{\mathrm{min}}$. observed quantity of $E M$. For example, mass-loss rates calculated according to Eq. (29) for the open H II regions characterized with $X=20$ are factor of about 2.5 larger than those estimated from Eq. (30). Most of the objects we investigated here contain extended nebulae which is mainly due to the high number of the ionizing photons they produce and a large separation between the binary components. Therefore for these objects we calculated $\dot{M}_{\mathrm{W}}$ according to Eq. (29) for $X=20$. In the cases of a less opened ionized zone (EG And, CQ Dra and possibly AX Per) we integrated Eq. (29) throughout its volume given by the parameter $X$. For EG And and CQ Dra, Skopal (2005) obtained $\dot{M}_{\mathrm{W}}$ and the corresponding parameter $X$ from accretion processes in these binaries. For AX Per Skopal et al. (2001a) determined $X=1.0$ from timing of the broad minimum observed during the 1958-72 quiescencent phase. However, as this value could change significantly (following the change of the LC profile), we present also a solution for $X=20$. Results of our analysis are given in the last column of Table 3 and Fig. 24 shows a comparison of our values with those determined by MNSV and Mikolajewska et al. (2002b) from 
radio observations as a function of the hot star luminosity. The graph confirms well the relationship between these two parameters. Mikolajewska et al. (2002b) discussed this correlation in terms of the illumination heating of the outer red giant's atmoshere, which enhances the mass-loss rate and consequently can induce a larger $L_{\mathrm{h}}$;

(ii) production of the nebular emission depends on the terminal velocity of the wind. A low-velocity wind corresponds to a higher concentration of emitters in the $\mathrm{H}$ II zone, which thus gives rise to the observed $E M$ for a lower mass-loss rate. We adopted $v_{\infty}=20 \mathrm{~km} \mathrm{~s}^{-1}$. Note that MNSV adopted $\mu \cdot v_{\infty}=10 \mathrm{~km} \mathrm{~s}^{-1}$ (i.e. $v_{\infty} \doteq 7 \mathrm{~km} \mathrm{~s}^{-1}$ ), which provides a factor of about 3 lower values of $\dot{M}_{\mathrm{W}}$;

(iii) there are other sources of the nebular radiation - e.g. emitters from the hot star wind and ionizations by collisions. The former accounts for about $10 \%$ of the total EM for well developed winds (e.g. AG Peg) and the latter can be important for nebulae with $T_{\mathrm{e}} \gtrsim 20000 \mathrm{~K}$. Therefore the emission measure produced solely by emitters of the giant's wind is lower than what we measure. From this point of view our estimates of $\dot{M}_{\mathrm{W}}$ represent upper limits as they were derived from the total $E M$. For example, the massloss rate derived independently from accretion process in CQ Dra $\left(\dot{M}_{\mathrm{W}}=8.2 \times 10^{-8} M_{\odot} \mathrm{yr}^{-1}\right.$, Skopal 2005) is by factor of $\sim 1.5$ lower than that derived here $\left(\dot{M}_{\mathrm{W}}=1.2 \times\right.$ $10^{-7} M_{\odot} \mathrm{yr}^{-1}$, Table 3).

Variability: the reconstructed SED at very different binary positions confirmed the orbitally-related variation in the emission measure which causes the well-known wave-like variation in the LCs as a function of the orbital phase. The nature of this type of variability was recently investigated by Skopal (2001a). Here we used his conversion between the observed $E M$ and the magnitude to reconstruct the $U$ LCs of RW Hya and SY Mus from the IUE spectra (Figs. 17 and 18). The observed amplitude of the wave-like variation is proportional to the ratio of fluxes from the nebula and the giant, which is a function of the wavelength - flux from the giant/nebula increases/decreses with increasing lambda. The contribution from the giant at the considered passband depends mainly on its spectral type, while the quantity of the nebular flux results from ionization/recombination processes and can be very different for individual objects. Figure 25 shows that the contribution from the giant in the $U B V$ bands is a function of its effective temperature, which determines the wavelength-dependence of the wave-like variation in LCs: yellow objects show $\Delta U / \Delta V \gg 1$, while those containing late-type M-giants have $\Delta U / \Delta V \sim 1-3$. In the column of the same $T_{\text {eff }}$, objects with a strong nebula are located at the bottom (i.e. $\Delta U / \Delta V \sim 1$ ). This is the case of V1329 Cyg at the column of $T_{\text {eff }}=3300 \mathrm{~K}$ and BFCyg among objects at $T_{\text {eff }}=3400 \mathrm{~K}$.

\subsubsection{Stellar continuum radiation}

The hot star temperature, $T_{\mathrm{h}}$ : it was not possible to determine directly this parameter with our fitting procedure for $T_{\mathrm{h}} \gtrsim 10^{5} \mathrm{~K}$. As a result we selected appropriate values of $T_{\mathrm{h}}$

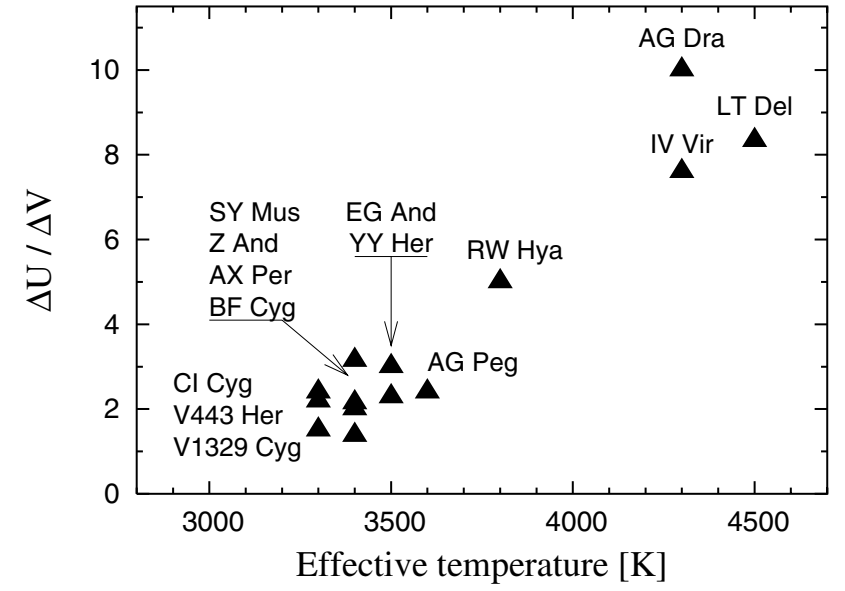

Fig. 25. Ratio of amplitudes in $U$ and $V$ of the orbitally-related variation in quiescent LCs as a function of the effective temperature of the giant. The ratio of fluxes from the giant and the nebula depends largely on the giant's effective temperature, which determines the overall relationship. Systems with a higher nebular emission have lower ratio of $\Delta U / \Delta V$ for the given $T_{\text {eff. }}$.

according to the ionizing capacity of the hot source and the observed emission measure:

(i) if the parameter $\delta<1$ for $T_{\mathrm{h}} \gtrsim 10^{5} \mathrm{~K}$ we adopted the Zanstra temperature for the ionizing object according to MNSV. This was the case of CICyg, SY Mus, and AGPeg. For two limiting cases, EG And(10/09/95) and BFCyg(10/04/92), we adopted the corresponding minimum temperatures, $T_{\mathrm{h}}^{\mathrm{min}}$, because the referred Zanstra temperatures were below this lower limit. Note that for $T_{\mathrm{h}}<T_{\mathrm{h}}^{\min }$ the ionizing capacity is not sufficient to produce $E M_{\mathrm{obs}}$. For RW Hya we adopted $T_{\mathrm{h}}=110000 \mathrm{~K}$, because its ionized zone is very open even for $T_{\mathrm{h}}=T_{\mathrm{h}}^{\min }=$ $80000 \mathrm{~K}$ and the suggested Zanstra temperature is below $T_{\mathrm{h}}^{\min }$. In this special case it was also possible to get this temperature directly by fitting the well exposed spectrum from 19/07/79;

(ii) if the parameter $\delta \sim 1$ for a certain temperature $T_{\mathrm{h}}>$ $10^{5} \mathrm{~K}$, i.e. $E M_{\mathrm{obs}}$ is very large, we adopted $T_{\mathrm{h}}=T_{\mathrm{h}}^{\min }$. Then the parameters derived from the SED, $L_{\mathrm{h}}$ and $\theta_{\mathrm{h}}$ represent their lower and upper limit, respectively ( $\mathrm{Z}$ And, V1329 Cyg, AG Dra, CQ Dra; Table 3);

(iii) for two cases, V443 Her and AX Per, it was possible to determine the $T_{\mathrm{h}}$ temperature directly as the fitting parameter of our procedure, because of its low value. However, a relatively strong nebular radiation in these systems yields the parameter $\delta \gg 1$. Therefore, in Table 3 we also give parameters for the case $\delta=1\left(T_{\mathrm{h}}=T_{\mathrm{h}}^{\mathrm{min}}\right)$.

The effective radius, $R_{\mathrm{h}}^{\text {eff }}$ : according to Eq. (6), $R_{\mathrm{h}}^{\text {eff }}$ is the radius of a sphere which has the same luminosity as the observed hot object seen under the angle $2 \theta_{\mathrm{h}}$. According to present knowledge, accretors in symbiotic binaries are white dwarfs of a mean mass $M_{\mathrm{WD}} \sim 0.6 M_{\odot}$ (Corradi et al. 2003). However, only in the case of CQDra, from our sample of the objects, is the $R_{\mathrm{h}}^{\mathrm{eff}}$ radius comparable to that of a white dwarf (Table 3 ). 


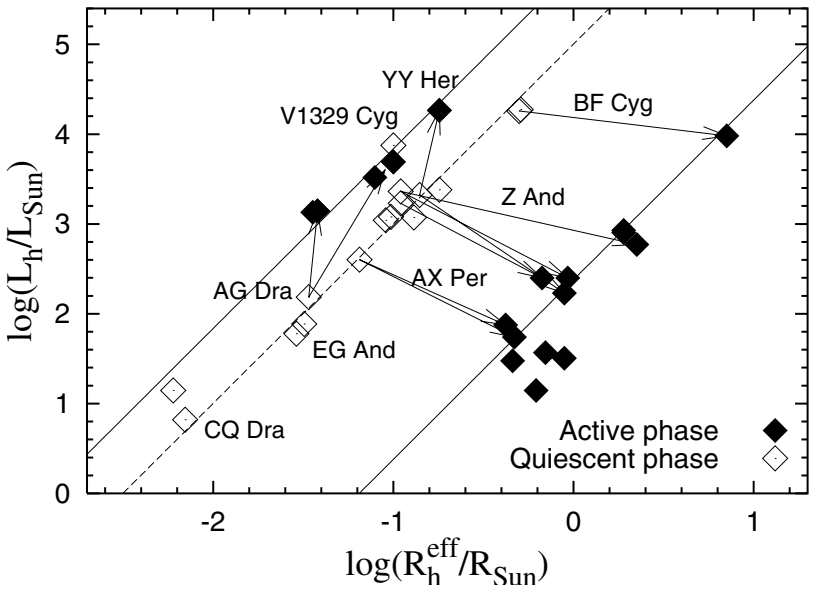

Fig. 26. The $L-R$ relationship for the hot stellar component of radiation. It satisfies well radiation of stellar photospheres (Eq. (31)). During quiescence (open squares, dashed line) stellar components of investigated objects radiate at a characteristic temperature of $\sim 103000 \mathrm{~K}$, while during outbursts (filled squares, solid lines) this temperature decreases significantly to $\sim 22000 \mathrm{~K}$ (1st-type of outbursts) or increases to $\sim 165000 \mathrm{~K}$ (2nd-type of outbursts).

Here the very small effective radius limits the white dwarf radius to $R_{\mathrm{WD}} \lesssim 0.006 R_{\odot}$, which implies a rather massive white dwarf, $M_{\mathrm{WD}} \sim 1 M_{\odot}$ (Hamada \& Salpeter 1961). Effective radii derived from the SED of other objects are a factor of about 10 (or more) larger than a typical white dwarf's radius. Qualitatively, this can be understood as a result of accretion process that creates an optically thick photosphere around the mass core. Below we investigate this possibility:

(i) The extreme cases of SEDs with $\delta \gtrsim 1$ (i.e. $T_{\mathrm{h}}<T_{\mathrm{h}}^{\min }$ ) suggest a disk-like structure of the hot source. The $o b$ served hot stellar radiation is not capable of giving rise to the strong nebula in the system which constrains that an unseen part of the hot source radiates at a higher temperature to produce the necessary surplus of the ionizing photons. Thus, the inner ionizing source of the hot object has to be obscured by the accreted matter more in directions forwards the observer than at its poles. The effect is significant for highly inclined systems.

(ii) Models corresponding to $\delta<1$. If the hot stellar source radiation mimics that of a stellar photosphere, then it has to satisfy the $L R T$ relation, which can be expressed in solar units as

$\log \left(\frac{L_{\mathrm{h}}}{L_{\odot}}\right)=2 \log \left(\frac{R_{\mathrm{h}}^{\mathrm{eff}}}{R_{\odot}}\right)+4 \log \left(\frac{T_{\mathrm{h}}}{T_{\odot}}\right)$.

A good agreement between this relation and our quantities of $L_{\mathrm{h}}$ and $R_{\mathrm{h}}^{\text {eff }}$ from Table 3 confirms the abovementioned suggestion (Fig. 26). The best fit gives $4 \log \left(T_{\mathrm{h}} / T_{\odot}\right)=$ $5.0 \pm 0.04$, i.e. they radiate at a characteristic temperature of $103000 \pm 2500 \mathrm{~K}$ for $T_{\odot}=5800 \mathrm{~K}$. Here, to get a better solution, we omitted the cases of V1329 Cyg and CQ Dra(11/10/86) (see Fig. 26). Thus we cannot observe just the accretor's surface.

Variability: observations at/around opposite conjunctions of the binary components confirmed the presence of an additional source of extinction, which attenuates the continuum (seemingly) at all wavelengths and is more pronounced at positions around the inferior conjunction of the giant (here $\mathrm{Z}$ And, V1329Cyg). MNSV first noticed this extinction process. The effect is known for RW Hya, SY Mus, BF Cyg (e.g. Dumm et al. 1999). Horne et al. (1994) modeled the influence of the absorbing medium to the ultraviolet, optical and near-infrared spectrum of of the white dwarf in the eclipsing dwarf nova OY Car. They found that besides deep features around 1600 and $2000 \AA$ the absorbing gas also produces a modest optical depth in the Balmer and Pashen continuum which makes it lower by about $20 \%$ in the ultraviolet (see their Fig. 8). The magnitude of this effect is probably a function of the orbital inclination. We did not find it in the spectra of AG Dra and AG Peg.

\subsection{The active phase}

\subsubsection{The high-temperature nebula}

The HTN is seen directly during optical eclipses of all investigated stars (BF Cyg, CH Cyg, CI Cyg, AR Pav and AX Per) as the only component of the hot object radiation in the UV spectrum. Out of eclipses it is indicated by a non-zero level of the Rayleigh attenuated far-UV continuum. The nebular nature of this component was proved directly by the optical spectrum containing the Balmer jump in emission taken simultaneously with the ultraviolet measurements of the strong 1993-outburst of YY Her (Fig. 16). Also simultaneous UV/optical/radio observations of BFCyg taken at the totality of the optical eclipse during its 1989-92 active phase suggest the nebular origin of this component of radiation. In the UV it is given by its flat profile (Fig. 8), in the optical by the very negative colour index, $U-B \sim-0.7$ (Skopal 1992), and in the radio the observed flux is increased to $0.84 \mathrm{mJy}$ with respect to precedent values (Seaquist et al. 1993). The only counterpart to the radio emission is the HTN in the ultraviolet, which thus confirms its nebular nature. Its profile corresponds to high electron temperatures, $T_{\mathrm{e}}>3 \times 10^{4} \mathrm{~K}$ (Table 4 ) and it is complementary to other components of radiation throughout the UV/optical/IR wavelengths to match the observed SED. These characteristics imply that the HTN-radiation is not subject to the Rayleigh scattering process and the corresponding ionized medium is not eclipsed by the giant. Thus a major fraction of the HTN region is located at least $\sim 1 R_{\mathrm{g}}$ above/below the orbital plane. The HTN appears to be very strong in the spectra from active phases. During transitions to quiescence its emission measure gradually decreases (see figures for Z And, CH Cyg, BF Cyg). Other IUE observations also support such evolution. For example, the HTN emission measure from AX Per spectra on 30/11/90 (Fig. 21) decreased by a factor of about 2.5 on its transition to quiescence on 04/09/94 (SWP52027 + LWP29094, $\varphi=0.01$ - not presented here). During quiescent phases it can be recognized as a very faint emission in UV (e.g. RW Hya, Fig. 17). Pereira et al. (1995) noted the presence of such the remaining light also during the eclipse of SY Mus. They assigned it most likely to nebular emission. This suggests that creation of a strong HTN region is connected with the mass-outflow from the central star during outbursts. Below we discuss its origin. 
(i) Photoionization of a rather massive hot star wind can be excluded, because this would produce a significant emission within about $100 R_{\odot}$ from its source. The emissivity of such the wind is proportional to the square of the particle concentration, which is diluted in the wind with the radial distance $r$ as $1 / r^{2}$. In addition, a small wind velocity at the vicinity of its source makes the particle density even higher, which significantly enhances the emissivity of the inner parts of the ionized wind (cf. Fig. C1 of Skopal et al. 2002a). If this were the case, we would have observed a significant decrease in the emission from the HTN during the total eclipse. This, however, is not the case.

(ii) Photoionization of the neutral particles from the giant's wind would produce nebular emission at $T_{\mathrm{e}} \sim 1-2 \times 10^{4} \mathrm{~K}$ as we observe during quiescent phases. However, this does not correspond to the HTN properties.

(iii) The ejected material can make the surrounding nebular medium more opaque, which could lead to a significant heating of the outer layers of the symbiotic nebula, because the ionizing radiation is modified by absorption in the nebula - the higher-energy photons penetrate into the gas at larger (i.e. more opaque) distances from the central star, and thus the mean energy of the produced electrons is higher there (Osterbrock 1974). The result probably depends strongly on the structure of the surrounding ionized material.

(iv) In the analogy to the colliding wind model (e.g. Girard \& Willson 1987; Nussbaumer \& Walder 1993), a high temperature collisionally-ionized shock layer, which can be ionized also by the radiation field of the hot star, can be created well above the accretion disk. The collisions are between the high-velocity hot star wind and the slowly moving particles of the cool giant wind and/or circumbinary material. The temperature of this region can reach well above $10^{6} \mathrm{~K}$. Problems here are connected mainly with the efficiency of cooling the hot plasma and properties of the driven hot star wind. Nevertheless, physical separation of the HTN from the central hot object, its very high electron temperature and production of the emission lines with very high ionization potential could be produced by this type of interaction.

\subsubsection{The low-temperature nebula}

The LTN is subject to eclipses, which determines its location in the vicinity of the central star and limits its linear size to $2 R_{\mathrm{g}}$. This case is reproduced by CICyg, AR Pav and AXPer, for which there are available ultraviolet observations taken in and out of their optical eclipses during activity. The radiative properties of the LTN are characterized by its emission measure and luminosity. They are determined by the fitting parameters $k_{\mathrm{N}}$, $T_{\mathrm{e}}$ and $\tilde{a}$ in relations (13) and (14). Its electron temperature is between 10000 and $18000 \mathrm{~K}$, which means that this region is predominately due to photoionization. Geometrical properties of the LTN - mainly its size and location - suggest its very high ionization degree. According to the ionization formula (e.g. Gurzadyan 1997), $N_{+} / N_{1} \sim$ a few $\times 10^{3}$ even for very high electron concentrations of $N_{\mathrm{e}} \sim$ a few $\times 10^{10} \mathrm{~cm}^{-3}$, because of a high value of the dilution factor (see below). Therefore and due to a small or negligible contribution from the doubly ionized helium ( $\tilde{a} \sim 0$ ) we can assume $N_{\mathrm{e}}=N_{\mathrm{H}^{+}}$. Further, for the temperature of the ionizing source $T_{\star} \equiv 10^{5} \mathrm{~K}$, the average electron temperature $T_{\mathrm{e}}=14000 \mathrm{~K}$, a dilution factor $W \doteq$ $\left(R_{\star} / R_{\mathrm{LTN}}\right)^{2} \sim(0.01 / 100)^{2}=10^{-8}$ and the optical depth of the nebula $\tau_{\mathrm{c}}=\kappa_{\mathrm{c}} N_{1} R_{\mathrm{LTN}} \sim 1\left(\kappa_{\mathrm{c}}=6.3 \times 10^{-18} \mathrm{~cm}^{2}\right.$ is the hydrogen absorption coefficient at the Lyman limit, $N_{1}$ is the concentration of neutral $\mathrm{H}$ atoms and for $R_{\mathrm{LTN}}$ we adopt $100 R_{\odot}$ as a maximum), the ionization formula provides the electron density

$N_{\mathrm{e}} \sim 10^{9} \mathrm{~cm}^{-3}$.

An independent estimate of $N_{\mathrm{e}}$ can be made from the size of the LTN (it is subject to eclipse) and its emission measure. If we approximate its volume by a sphere of radius $R_{\mathrm{LTN}} \equiv 100 R_{\odot}$ then its emitting volume $V_{\mathrm{LTN}}=1.4 \times 10^{39} \mathrm{~cm}^{3}$. Consequently, the emission measure obtained from the spectra out of eclipses, $E M_{\mathrm{LTN}}=N_{\mathrm{e}}^{2} V_{\mathrm{LTN}}=10^{58}-10^{60} \mathrm{~cm}^{-3}$, yields the average electron concentration

$N_{\mathrm{e}} \sim$ a few $\times 10^{9} \mathrm{~cm}^{-3}$,

which is comparable with the estimate made above using the ionization formula. This agreement supports that the LTN is mainly due to photoionization. Kenyon et al. (1991), based on an analysis of the line emitting regions in CICyg, also suggested the presence of two nebular regions in this system a compact nebula located within the Roche lobe of the accretor and a low density, collisionally-ionized nebula having a form of bipolar streams above/below the disk's surface.

\subsubsection{The hot stellar source}

The region of the HSS is subject to eclipses (Figs. 8-10, 19 and 21). Its radiation can be approximated by that of a black body at low temperatures. Below we summarize its radiative and geometrical properties as follows:

1. Figure 26 shows: (i) the HSS radiation of most of objects satisfies that of a stellar photosphere at a characteristic temperature of $\sim 22800 \mathrm{~K}$. The HSS of CHCyg and TX CVn radiates even at lower temperatures; we did not include them in the fit in Fig. 26; (ii) there is a significant enlargement of the $R_{\mathrm{h}}^{\text {eff }}$ radius by a factor of $\sim 10$ with respect to quantities from quiescence. This and the simultaneous presence of the two-temperature-type of the UV spectrum (see bellow, Sect. 5.3.4) suggest that the optically thick medium expands at the orbital plane in the form of a disk; (iii) the observed luminosity of the HSS during the 1st-type of outbursts (see below; Fig. 26) decreases by a factor of about 2-10 with respect to the hot object luminosity during quiescence (Tables 3 and 4). This is because the HSS, which develops during these outbursts, blocks a fraction of radiation from the centre in the direction to the observer. We discuss this situation in Sect. 5.3.6 in more detail.

2. The HSS radiation is attenuated by the Rayleigh scattering process for all the well-known eclipsing systems presented here (BF Cyg, CH Cyg, CI Cyg, AR Pav and AX Per; 
Table 4). The effect is measured at any orbital phase. We therefore believe that the absorbing gas is located at the outer rim of the disk. On the other hand the presence of the Rayleigh-attenuated far-UV continuum in Z And and TXCVn confirms the recent suggestion of their high orbital inclination (Skopal 2003a; Skopal et al. 2004), and thus suggests it also for $\mathrm{CD}-43^{\circ} 14304$ (Fig. 5). Generally, all the orbits of the objects showing the two-temperaturetype of UV spectrum during activity should be highly inclined to the observer. Here this applies to AE Ara (Fig. 4).

3. Outbursts of AGDra and the major 1993-outburst of YY Her differ significantly from those described above. A strong nebular and very hot stellar components of radiation developed immediately at the beginning of these outbursts. The hot stellar radiation is characterized by a higher temperature $T_{\mathrm{h}} \sim 165000 \mathrm{~K}$, a larger $L_{\mathrm{h}}$ and smaller $R_{\mathrm{h}}^{\text {eff }}$ with respect to values from quiescence (Fig. 26). The case of AGDra could be caused mainly by an inclination effect: this system is seen more from its pole $\left(i=60 \pm 8^{\circ}\right.$, Schmid $\&$ Schild 1997) and thus the central ionizing source can be directly observed. As a result both a very hot and luminous stellar and strong nebular component of radiation can be measured directly. The observed large difference in the HSS temperature during the minor 1981 and the major 1993 outburst of YY Her (Fig. 16) is probably related to different properties of the mass outflow. Material during the major outburst was ejected probably at very high velocity, which precluded formation of a cool stellar pseudophotosphere. As a result the simultaneous massive injection of new particles (emitters) by the active star and the increase of its ionizing capacity enhanced the nebular emission by a factor of 20 (Table 4).

Accordingly, these observational properties of the HSS during active phases suggest two types of outbursts: the 1st type is characterized by a larger $R_{\mathrm{h}}^{\text {eff }}$ and lower $T_{\mathrm{h}}$ (points 1 and 2 above) and the 2 nd type is determined by a smaller $R_{\mathrm{h}}^{\text {eff }}$ and higher $T_{\mathrm{h}}$ (the point 3 above) with respect to their quiescent values (Fig. 26). All the active objects with a high orbital inclination show characteristics of the 1st type of outbursts. González-Riestra et al. (1999) identified "cool" and "hot" outbursts of AGDra. They lie within the 2nd type of outbursts. According to the point 1. (iii) above one can speculate that the 1st-type of outbursts represent a special case of the 2nd-type due to a high orbital inclination (Sect. 5.3.6). Finally, we note that a large number of objects with highly inclined orbits, among those intensively investigated, reflects a systematic selection, because the symbiotic phenomenon is best recognizable for such systems.

\subsubsection{The two-temperature UV spectrum}

The presence of the HTN, LTN and a warm HSS components of radiation determines the two-temperature-type of the ultraviolet spectrum. In our approach, the cool spectrum is produced by the warm HSS radiation $(\sim 22000 \mathrm{~K})$ and the hot spectrum is represented by the emission from the HTN+LTN powered by an unseen hot ionizing source $\left(\gtrsim 10^{5} \mathrm{~K}\right)$ in the system. Superposition of these components results in the observed, more or less flat ultraviolet continuum. The far-UV region is dominated by the HSS radiation, while the near-UV continuum often contains a large contribution from the LTN. The slope of the HTN radiation throughout the ultraviolet is very small. This type of the UV spectrum represents a common feature of active phases classified here as the 1st type.

\subsubsection{Basic structure of the hot active object}

Radiative and geometrical properties of individual components of radiation observed during the 1st type of outbursts allow us to reconstruct a basic configuration of the hot active object. The following points are relevant.

(i) The two-temperature-type of the UV spectrum suggests the presence of an optically thick disk-like torus around the hot star, which is seen approximately edge-on. The disk's outer rim has to be flared sufficiently to occult permanently the central source of the hot ionizing radiation, which is not seen directly in the spectrum. Under such conditions, the energetic radiation from the central source is absorbed and diffused by the disk body in the direction to the observer. At the distance $R_{\mathrm{HSS}}$ from the center, it releases the disk at much lower temperature ( $22000 \mathrm{~K})$. We identify this warmer pseudophotosphere as the HSS. The HSS radiation then continues throughout the neutral gas surrounding the accretor beyond the disk, which attenuates its amount by the Rayleigh scattering process and affects (often drastically) its profile by numerous absorptions of mainly Fe II lines, the so-called "iron curtain".

(ii) The geometrical and physical properties of the LTN localize this region above/below the disk plane approximately within the linear size of the HSS (it is also subject to eclipse). It is probably also flared at the outer edge of the disk, because directions from the hot inner parts of the disk to poles have to be rather free for high-velocity particles and ionizing photons, which give rise the uneclipsed emission from the HTN. In addition, the LTN has to "see" well the ionizing radiation from the hot center. This view is supported by theoretical modeling of Plavec \& Hubený (1994), who found that the vertical outer edge of accretion disks in symbiotic binaries can be extremely extended. In the real case the vertical extension is limited by the eclipsing nature of the LTN. For the sake of simplicity, we draw schematically the LTN vertical dimension as a linear function of the disk radius (cf. Fig. 27).

(iii) The HTN region has to be located high enough above/below the disk ( $>1 R_{\mathrm{g}}$ ) not to be eclipsed by the giant (e.g. Figs. 10 and 19). However, our observations were not sufficient to determine its geometry more accurately. The HTN can be in part collisionaly ionized, which suggests that a mechanisms similar to that of the colliding wind model could be responsible for its rise (Sect. 5.3.1).

A sketch of the above described geometrical structure of the hot object in symbiotic binaries during the 1st-type of outbursts is shown in Fig. 27. 

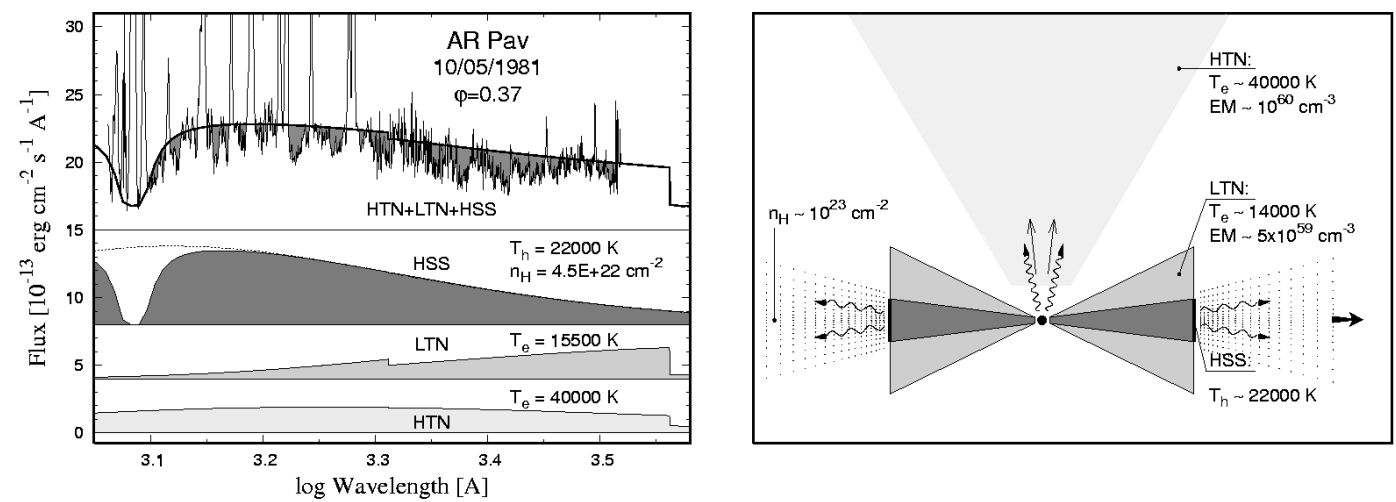

Fig. 27. Schematic representation of a basic structure of hot objects during outbursts of the 1st-type. The left panel shows example of individual components of radiation we isolated from the AR Pav spectrum observed on 10/05/81. Note that the HSS radiation is Rayleigh attenuated at the far-UV region and veiled by a forest of blended Fe II absorptions. The right panel shows a sketch of the corresponding emitting regions as seen on a cut perpendicular to the orbital plane containing the accretor. During outbursts the disk's outer rim has to be flared to occult permanently the central source of the hot ionizing radiation. The model is described in Sect. 5.3.5.

\subsubsection{Luminosity of the hot active object}

In this section we discuss the total luminosity of hot objects undergoing the 1st-type of outbursts. The edge-on disk/torus, which develops during these outbursts, blocks a fraction of radiation from the accretor in the direction to the observer. We measure directly only its part in the form of the HSS radiation. The remaining part of the accretor's luminosity can become visible due to its interaction with the medium surrounding the hot object. We detect only its fraction converted into the nebular emission in the form of the LTN and HTN radiation. However, a part of the accretor's luminosity, $L_{\text {out }}$, can escape the system. Therefore we write the bolometric luminosity, $L_{\mathrm{T}}$, produced at/around the white dwarf as

$L_{\mathrm{T}}=L_{\mathrm{HSS}}+L_{\mathrm{HTN}}+L_{\mathrm{LTN}}+L_{\mathrm{out}}$.

The not-detectable light includes the radiation, which is not capable of ionizing the surrounding medium (for $T_{\mathrm{h}}=10^{5} \mathrm{~K}$ and hydrogen it is about $10 \%$ of the total radiation, Fig. 2 of Skopal $2003 \mathrm{~b}$ ), and a part of ionizing photons, which are not converted into the nebular emission. Thus we, generally, observe

$L_{\mathrm{h}}^{\mathrm{obs}} \ll L_{\mathrm{T}}$.

This is the case of all systems showing signatures of the 1st type of outbursts (compare data from Tables 3, 4 and Fig. 26). On the other hand when viewing the system more pole-on we can detect directly all the radiation released during outbursts. An example here is AG Dra. During its "hot" outbursts (Sect. 4.13) we have

$L_{\mathrm{h}}^{\mathrm{obs}} \approx L_{\mathrm{T}} \equiv L_{\mathrm{Q}}$

if one adopts the total luminosity as that from quiescence, $L_{\mathrm{Q}}$. Note that for $T_{\mathrm{h}}>T_{\mathrm{h}}^{\min }$ one can easily get equivalence between the luminosities in the above relation. This suggests an accretion-powered nature of these outbursts. However, for "cool" outbursts we measure

$L_{\mathrm{h}}^{\mathrm{obs}} \gg L_{\mathrm{Q}}$, which signals an additional source of the energy during these events, probably from a TNR. Some details on the nova-like eruptions of symbiotic stars can be found in Mikolajewska \& Kenyon (1992b).

\subsection{Comparison with previous models}

A modeling of the composite continuum for a large sample of well-studied symbiotic stars similar to the present one has been carried out by MNSV and KW. The basic conceptional differences between these and our approach were introduced in the Sect. 1. Here we present a concise summary of differences between our models and those suggested by other authors. The following points are relevant:

(i) Our SED model (Eq. (13)) is less sophisticated than that suggested by MNSV. We did not include a geometry of radiating regions and consider just basic sources of radiation and effects that can be recognized by observations. This simplifies significantly modeling the SED and makes it more accessible for a wider community. On the other hand, this does not result in decisive differencies in $R_{\mathrm{h}}^{\text {eff }}$ and $L_{\mathrm{h}}$ from quiescent phases.

(ii) In our approach the electron temperature represents the fitting parameter, while MNSV considered it to be within a small range (Sect. 1). This revealed drastic variations in $T_{\mathrm{e}}$ even for the same object (e.g. Z And, AG Peg). This allowed us to determine corresponding EMs more accurately. We note that an adoption of a "typical" value of $T_{\mathrm{e}}$ can result in an incorrect SED (e.g. Fig. 4 in Munari et al. 1997a).

(iii) In our approach we used temperatures required by the basic photoionization model (i.e. $T_{\mathrm{h}}^{\mathrm{min}}$ (Eq. (23)) or $T_{\text {Zanstra }}$, see Sect. 5.2.2) as nebulae during quiescent phases are largely radiatively ionized $\left(T_{\mathrm{e}} \lesssim 20000 \mathrm{~K}\right)$. In cases of AX Per and V443 Her a large temperature difference, $\Delta T \equiv T_{\mathrm{h}}^{\mathrm{min}}-T_{\mathrm{fit}} \sim 35000 \mathrm{~K}$ (Table 3 ), suggests the presence of a disk-like structured material around their 
accretors even during quiescent phases (Sects. 4.15 and 4.21). MNSV discarded the presence of accretion disks in their solutions, whereas KW considered them as a reasonable possibility for some cases. Most recently Sion et al. (2002), Sion (2003) and Kolb et al. (2004) applied model atmospheres in spectral analyses of the hot stellar component of radiation from RW Hya, AGDra and EG And. They also indicated significantly lower effective temperatures than those given by conditions of photoionization in these systems. Effective temperatures of their models and our $T_{\mathrm{h}}^{\mathrm{min}}$ for EG And and RW Hya (Table 3) give a similar $\Delta T$ to what we found for V443Her and AXPer. This suggests that we detect temperatures of different regions in the hot object. Within our interpretation we measure directly a cooler pseudophotosphere ( edge-on disk-like structured accreting material), while the underlying hotter accretor's surface can be "seen" only by the surrounding medium, which converts its radiation to the nebular emission, based on whose properties we can derive indirectly the temperature of the hottest part of the ionizing source as $T_{\text {Zanstra }}$ and/or $T_{\mathrm{h}}^{\mathrm{min}}$. Such a structure of hot components can explain the apparent inconsistence between effective temperatures from model atmospheres analyses and those required by the observed nebular emission. In accordance with this view the temperature difference $\Delta T$ should be larger for objects with a high orbital inclination and large mass-loss rates, and vice versa. This idea could be tested by applying the model atmosphere spectral analysis to objects seen rather pole-on and with a small $\dot{M}_{\mathrm{W}}$ (e.g. CQ Dra in our sample). Such testing is beyond the possibilities of our simple black-body fitting.

(iv) The mass-loss rate was calculated for a more realistic structure of the giant's wind. We considered a $\beta$-law wind, whereas MNSV adopted a constant wind. In addition, we used a more accurately detemined EMs (the point (ii) above). This difference, however, did not yield a large quantitative effect. Mikolajewska et al. (2002b) estimated $\dot{M}_{\mathrm{W}}$ from their radio $\mathrm{mm} / \mathrm{sub}$-mm observations assuming a partially ionized red giant wind determined by opened ionized zones. This approach is, in principle, similar to our calculations. A comparison of our $\dot{M}_{\mathrm{W}}$ quantities with those mentioned above is satisfactory (Fig. 24).

(v) We quantified contributions from cool components by comparing an appropriate synthetic spectrum to IR fluxpoints given by photometric measurements. KW reconstructed the spectrum of cool components according to energy distributions of Johnson (1966) and Lee (1970) and scaled it to absolute $V$ magnitudes of K3, M 2 and M 4 giants. MNSV restricted their analysis only to UV spectra. Our approach allowed us to estimate effective temperatures and angular radii of giants independently from their spectral types. Application of synthetic spectra of red/yellow giants is of a particular importance to model SEDs for objects with a considerable cool component contribution at the near-UV (yellow symbiotics and those with a small nebular emission, e.g. CQDra) to get a correct $T_{\mathrm{e}}$ and $E M$. For comparison, a simple blackbody fit gives a large contribution at these wavelengths (e.g. Fig. 7 in Skopal et al. 2000).

(vi) A major qualitative difference is that we modeled spectra from all the quiescent phases, active phases and eclipses, whereas MNSV restricted their analysis exclusively to quiescent phases at binary positions well outside eclipses. Their trials to model active phases failed (Fig. 8 and Table 6 in MNSV). Application of our method to spectra taken during active phases revealed their common properties (Sect. 5.3; Fig. 27). This represents the main novelty of the present paper. For comparison, in our model of the active hot object of the 1st-type the flared disk permanently occults its hotter inner parts in the line-of-sight, whereas according to previous suggestions (e.g. KW; Kenyon et al. 1991; Mikolajewska \& Kenyon 1992a) we observe radiation from the inclined disk's surface including its central hot parts. However, fits to the observed continuum by using parameters of the latter models are poor (e.g. Fig. 9 in MNSV). A quantitative application of standard disk models to the UV/optical/IR continuum was carried out by Skopal (2003b) for AR Pav. This approach failed to explain the UV continuum with the disk and its boundary layer.

(vii) To select the continuum flux-points we considered the influence of the iron curtain. This led to determination of more realistic model parameters for spectra from mainly active phases. Particularly, we obtained solution also for $\mathrm{CHCyg}$, on which approaches of both the groups (KW and MNSV) failed. A first suggestion was made by Shore \& Aufdenberg (1993) for the recurrent nova $\mathrm{T} \mathrm{CrB}$. However, ignoring the effect of the iron curtain for this case, Stanishev et al. (2004) obtained an ineffectual SED corresponding to false parameters, which are not consistent with the observed hot object luminosity. Also Skopal (2003a) obtained false quantities of $\mathrm{He}^{++}$abundance and $T_{\mathrm{e}}$ of the LTN in his modeling the $\mathrm{Z}$ And spectra from the active phase.

(viii) Finally, the presented comprehensive atlas of modeled and observed SEDs for intensively studied S-type symbiotic stars accompanied by LCs represents a large source of information for future works.

\section{Conclusions}

We introduced a method of disentangling the composite spectrum consisting of two stellar components of radiation (from cool giants and hot objects in the binary) and nebular radiation. We applied the method to $21 \mathrm{~S}$-type symbiotic binaries to isolate individual radiative components from their $0.12-5 \mu \mathrm{m}$ SED in the continuum during quiescent as well as active phases. The modeled continuum is determined by 8 fitting parameters: Temperatures, $T_{\mathrm{eff}}, T_{\mathrm{h}}$ and $T_{\mathrm{e}}$, angular radii, $\theta_{\mathrm{g}}$ and $\theta_{\mathrm{h}}$, scaling of the nebular emission, $k_{\mathrm{N}}$, hydrogen column density, $n_{\mathrm{H}}$ and average abundance of doubly ionized helium, $\tilde{a}$ (Sect. 3.1). These parameters then determine luminosities and 
radii of the stellar components and the emission measure of the nebula. Common properties of individual radiative components and their sources can be summarized as follows:

1. Radiation from the giant: (i) the SED of giants is defined by the fitting parameters, $T_{\text {eff }}$ and $\theta_{\mathrm{g}}$. Those having independently-determined radii (11 objects from our sample, Fig. 23) then provide the distance from their $\theta_{\mathrm{g}}$. Relations (27) and (28) show that their characteristics satisfy best those of normal giants. This confirms a generally accepted view that cool components in S-type systems are normal giants as given by their spectral types (e.g. Mürset \& Schmid 1999); (ii) the effective temperature of giants is critical for the amplitudes of the wave-like orbitally-related variation in the LCs (Fig. 25).

2. Hot object radiation during quiescence: (i) the nebulae radiate at a mean temperature of $19000 \mathrm{~K}$. By comparing the measured and calculated emission measure we derived the mass-loss rate from giants via their wind as $\dot{M}_{\mathrm{W}}=$ a few $\times 10^{-7} M_{\odot} \mathrm{yr}^{-1}$ (Table 3, Fig. 24); (ii) stellar continuum radiation determines the effective radii of the hot objects to be a factor of $\sim 10$ larger than the accretors. We thus observe pseudophotospheres due to the accretion process (Fig. 26). The extreme cases, for which the SED suggests $T_{\mathrm{h}}<T_{\mathrm{h}}^{\mathrm{min}}$ (the observed hot source is not capable of giving rise to the nebular emission), signal the presence of a disk-like structure of the circumstellar matter around the accretor. This is in agreement with theoretical modeling of accretion from the giant's wind that predicts formation of a stable, permanent disk around the accretor (Mastrodemos \& Morris 1998).

3. Hot object radiation during activity: (i) the stellar radiation satisfies that of a black-body photosphere. There are two types of outbursts: the 1st-type with the HSS radiating at significantly lower temperatures than during quiescence and the 2nd-type with higher characteristic temperatures (cf. Fig. 26). All the active objects with a high orbital inclination show features of the 1st type of outbursts: Rayleigh attenuation at any orbital phase, a strong influence from the iron curtain absorptions, a low characteristic temperature of $\sim 22000 \mathrm{~K}$, strong HTN emission, two-temperature-type UV spectrum and enlargement of $R_{\mathrm{h}}^{\text {eff }}$ by a factor of $\sim 10$ with respect to the quiescent values. The last two characteristics suggest an expansion of an optically thick medium at the orbital plane in the form of a disk; (ii) the low-temperature nebula (LTN) radiates at a mean electron temperature of $14000 \mathrm{~K}$. This region is subject to eclipses, which restricts its maximum linear radius to $2 R_{\mathrm{g}}$. Its emission measure, $10^{58}-10^{60} \mathrm{~cm}^{-3}$, suggests the electron concentration $N_{\mathrm{e}} \sim$ a few $\times 10^{9} \mathrm{~cm}^{-3}$; (iii) the high-temperature nebula (HTN) is characterized by $T_{\mathrm{e}}>30000 \mathrm{~K}$ and its major fraction is located well away from the hot object, not subject to eclipses. Its emission measure is between $10^{58}-10^{61} \mathrm{~cm}^{-3}$. Its origin could be within a scenario of the colliding wind model.

The characteristics of the UV-continuum and its components we observe during the 1 st type of outbursts can be interpreted in terms of an edge-on flared disk surrounded by the neutral material at the orbital plane and the nebulae located above/below its surface. The model is described in Sect. 5.3.5 and in Fig. 27.

Acknowledgements. The author is grateful to the anonymous referees for several helpful comments. Special thanks are due to Ivan Hubený for his constructive comments on the iron curtain and providing his code CIRCUS. The author would also like to thank Albert Jones for providing his visual light curves of AE Ara, CD - 43 14304 and RW Hya and Nikolai Tomov for his unpublished photometry of AGPeg in the $U$ band for this paper. The first parts of this research were done within the project No. SLA/1039115 of the Alexander von Humboldt foundation (from 1998), throughout the bilateral research project between the Royal Society of Great Britain and the Slovak Academy of Sciences. Early versions of this work were presented at workshops in Göttingen (2001), Los Cancajos (2002), Dubrovník (2003) and Strasbourg (2004). The final work on this project was partly supported by the Slovak Academy of Sciences under grant No. 2/4014/4.

\section{References}

Allen, D. A., \& Glass, I. S. 1974, MNRAS, 167, 337

Aller, L. H. 1954, Pub. DAO Victoria, 9, 321

Archipova, V. P., Ikonnikova, N. P., \& Noskova, R. I. 1995, Pisma v AZh, 21, 379

Belczyński, K., \& Mikolajewska, J. 1998, MNRAS, 296, 77

van Belle, G. T., Lane, B. F., Thompson, R. R., et al. 1999, AJ, 117, 521

Belyakina, T. S. 1992, Izv. Krymsk. Astrofiz. Obs., 84, 49

Bessell, M. S. 1983, PASP, 95, 480

Birriel, J. J., Espey, B. R., \& Schutle-Ladbeck, R. E. 2000, ApJ, 545, 1020

Bode, M. 2003, in Symbiotic Stars Probing Stellar Evolution, ed. R. L. M. Corradi, J. Mikolajewska, \& T. J. Mahoney, ASP Conf. Ser., 303, 359

Cannizzo, J. K., \& Kenyon, S. J. 1992, ApJ, 386, L17

Cardelli, J. A., Clayton, G. C., \& Mathis, J. S. 1989, ApJ, 345, 245

Castor, J. I., Abbott, D. C., \& Klein, R. I. 1975, ApJ, 195, 157

Corradi, R. L. M., Mikolajewska, J., \& Mahoney, T. J. 2003, Symbiotic Stars Probing Stellar Evolution, ASP Conf. Ser., 303 (San Francisco: ASP)

Crocker, M. M., Davis, R. J., Eyres, S. P. S., et al. 2001, MNRAS, 326, 781

Dobrzycka, D., Kenyon, S. J., \& Mikolajewska, J. 1993, AJ, 106, 284

Dumm, T., \& Schild, H. 1998, New Astron., 3, 137

Dumm, T., Schmutz, W., Schild, H., \& Nussbaumer, H. 1999, A\&A, 349, 169

Eyres, S. P. S., Bode, M. F., \& Skopal, A. 2002, MNRAS, 335, 526

Feast, M. W., Robertson, B. S. C., \& Catchpole, R. M. 1977, MNRAS, 179, 499

Fernández-Castro, T., Cassatella, A., Giménez, A., \& Viotti, R. 1988, ApJ, 324, 1016

Fernández-Castro, T., Gonzales-Riestra, R., Cassatella, A., \& Fuensalida, J. J. 1990, A\&A, 227, 422

Fekel, F. C., Joyce, R. R., Hinkle, K. H., \& Skrutskie, M. 2000, AJ, 119,1375

Fekel, F. C., Hinkle, K. H., Joyce, R. R., \& Skrutskie, M. 2001, AJ, 121, 2219

Gális, R., Hric, L., Friedjung, M., \& Petrík, K. 1999, A\&A, 348, 533 
Girard, T., \& Willson, L. A. 1987, A\&A, 183, 247

Glass, I. S., \& Webster, B. L. 1973, MNRAS, 165, 77

González-Riestra, R., Viotti, R., Iijima, T., \& Greiner, J. 1999, A\&A, 347,478

Gurzadyan, G. A. 1997, The Physics and Dynamics of Planetary Nebulae (Berlin: Springer-Verlag), 80

Gutiérrez-Moreno, A., Moreno, H., \& Costa, E. 1999, PASP, 111, 571

Hamada, T., \& Salpeter, E. E. 1961, ApJ, 134, 683

Hauschildt, P. H., Allard, F., Ferguson, J., Baron, E., \& Alexander, D. R. 1999, ApJ, 525, 871

Hinkle, K. H, Fekel, F. C., Johnson, D. S., \& Scharlach, W. W. G. 1993, AJ, 105, 1074

Horne, K., Marsh, T. R., Cheng, F. H., Hubený, I., \& Lanz, T. 1994, ApJ, 426, 294

Hric, L., Skopal, A., Chochol, D., et al. 1994, Contrib. Astron. Obs. Skalnate Pleso, 24, 31

Hric, L., Petrík, K., Niarchos, P., Velič, Z., \& Gális, R. 2001, IBVS, 5046

Iijima, T. 1988, Ap\&SS, 150, 235

Ikeda, Y., \& Tamura, S., 2000, PASJ, 52, 589

Isliker, H., Nussbaumer, H., \& Vogel, M. 1989, A\&A, 219, 271

Johnson, H. L. 1966, ARA\&A, 4, 193

Kamath, U. S, \& Ashok, N. M. 1999, A\&AS, 135, 199

Kenyon, S. J., \& Webbink, R. F. 1984, ApJ, 279, 252 (KW)

Kenyon, S. J., \& Garcia, M. R. 1989, AJ, 97, 194

Kenyon, S. J., \& Mikolajewska, J. 1995, AJ, 110, 391

Kenyon, S. J., Oliversen, N. A., Mikolajewska, J., et al. 1991, AJ, 101, 637

Kenyon, S. J., Mikolajewska, J., Mikolajewski, M., Polidan, R. S., \& Slovak, M. H. 1993, AJ, 106, 1573

Kolb, K., Miller, J., Sion, E. M., \& Mikolajewska, J. 2004, AJ, 128, 1790

Kolotilov, E. A., \& Yudin, B. F. 1994, Pisma v AZh, 20, 411

Kolotilov, E. A., Munari, U., \& Yudin, B. F. 1995, A\&A, 293, 815

Komárek, Z. 1990, Bull. Astr. Inst. Czechosl., 41, 131

Lee, T. A. 1970, ApJ, 162, 217

Lee, H-W. 2000, ApJ, 541, L25

Leedjärv, L., Burmeister, M., Mikolajewski, M., et al. 2004, A\&A, 415,273

Leitherer, C. 1988, ApJ, 326, 356

Léna, P., Lebrun, F., \& Mignard, F. 1999, Observational Astrophysics (Berlin: Springer-Verlag)

Livio, M., \& Warner, B. 1984, Observatory, 104, 152

Lorenzetti, D., Saraceno, P., \& Strafela, F. 1985, ApJ, 298, 350

Luthardt, R. 1984, IBVS, 2495

Luthardt, R. 1989, Veroff. Sterne Sonneberg, 10, 255

Mastrodemos, N., \& Morris, M. 1998, ApJ, 497, 303

Mikolajewska, J., \& Kenyon, S. J. 1992a, AJ, 103, 579

Mikolajewska, J., \& Kenyon, S. J. 1992b, MNRAS, 256, 177

Mikolajewska, J., \& Kenyon, S. J. 1996, AJ, 112, 1659

Mikolajewska, J., Kenyon, S. J., Mikolajewski, M., Garcia, M. R., \& Polidan, R. S. 1995, AJ, 109, 1289

Mikolajewska, J., Kolotilov, E. A., Shugarov, S. Yu., \& Yudin, B. F. 2002a, A\&A, 392, 197

Mikolajewska, J., Ivison, R. J., \& Omont, A. 2002b, Adv. Space Res., 30, 2045

Mikolajewska, J., Quiroga, C., Brandi, E., et al. 2003, in Symbiotic Stars Probing Stellar Evolution, ed. R. L. M. Corradi, J. Mikolajewska, \& T. J. Mahoney, ASP Conf. Ser., 303, 147

Munari, U., \& Buson, L. M. 1992, A\&A, 255, 158

Munari, U., \& Zwitter, T. 2002, A\&A, 383, 188

Munari, U., Margoni, R., \& Mammano, A. 1988, A\&A, 202, 83
Munari, U., Yudin, B. F., Taranova, O. G., et al. 1992, A\&AS, 93, 383 Munari, U., Kolotilov, E. A., Popova, A. A., \& Yudin, B. F. 1997a, Astron. Rep., 41, 802

Munari, U., Rejkuba, M., Hazen, M., et al. 1997b, A\&A, 323, 113

Mürset, U., \& Nussbaumer, H. 1994, A\&A, 282, 586

Mürset, U., \& Schmid, H. M. 1999, A\&AS, 137, 473

Mürset, U., Nussbaumer, H., Schmid, H. M., \& Vogel, M. 1991, A\&A, 248, 458 (MNSV)

Niehues, M., Bruch, A., \& Duerbeck, H. W. 1992, Messenger, 67, 38

Nussbaumer, H., \& Vogel, M. 1989, A\&A, 213, 137

Nussbaumer H., \& Vogel, M. 1991, A\&A, 248, 81

Nussbaumer, H., \& Walder, R. 1993, A\&A, 278, 209

Nussbaumer, H., Schmid, H. M., \& Vogel, M. 1989, A\&A, 211, L27

Ogley, R. N., Chaty, S., Crocker, M. M., et al. 2002, MNRAS, 330, 772

Oliversen, N. A., Anderson, C. M., Stencel, R. E., \& Slovak, M. H. 1985, ApJ, 295, 620

Osterbrock, D. E. 1974, Astrophysics of Gaseous Nebulae (San Francisco: W. H. Freeman and Company Press)

Pereira, C. B., \& Mello, G. F. P. 1997, AJ, 114, 2128

Pereira, C. B., Vogel, M., \& Nussbaumer, H. 1995, A\&A, 293, 783

Pereira, C. B., Smith, V. V., \& Cunha, K. 1998, AJ, 116, 1977

Perryman, M. A. C., Lindegren, L., Kovalevsky, J., et al. 1997, A\&A, 323, L49

Plavec, M. J., \& Hubený, I. 1994, in Interacting Binary Stars, ed. A. Shafter, ASP Conf. Ser., 56, 87

Quiroga, C., Mikolajewska, J., Brandi, E., Ferrer, O., \& García, L. 2002, A\&A, 387, 139

Reimers, D., Griffin, R. F., \& Brown, A. 1988, A\&A, 193, 180

Schild, H., \& Schmid, H. M. 1997, A\&A, 324, 606

Schild, H., Mürset, U., \& Schmutz, W. 1996, A\&A, 306, 477

Schild, H., Dumm, T., Mürset, U., et al. 2001, A\&A, 366, 972

Schmid, H. M., \& Nussbaumer, H. 1993, A\&A, 268, 159

Schmid, H. M., \& Schild, H. 1997, A\&A, 321, 791

Schmid, H. M., Dumm, T., Mürset, U., et al. 1998, A\&A, 329, 986

Schmutz, W., Schild, H., Mürset, U., \& Schmid, H. M. 1994, A\&A, 288,819

Schröder, K.-P. 1985, A\&A, 147, 103

Seaquist, E. R., Taylor, A. R., \& Button, S. 1984, ApJ, 284, 202 (STB)

Seaquist, E. R., Krogulec, M., \& Taylor, A. R. 1993, ApJ, 410, 260

Selvelli, P. L., Cassatella, A., \& Gilmozzi, R. 1992, ApJ, 393, 289

Shore, S. N., \& Aufdenberg, J. P. 1993, ApJ, 416, 355

Sion, E. M. 2003, in Symbiotic Stars Probing Stellar Evolution, ed. R. L. M. Corradi, J. Mikolajewska, \& T. J. Mahoney, ASP Conf. Ser., 303, 193

Sion, E. M., Mikolajewska, M., Bambeck, D., \& Dumm, T. 2002, AJ, 123,983

Sokoloski, J. L., Kenyon, S. J., Kong, Ak. H., et al. 2002, in The Physics of Cataclysmic Variables and Related Objects, ed. B. T. Gänsicke, K. Beuermann, \& K. Reinsch, ASP Conf. Ser., 261, 667

Skopal, A. 1991, IBVS, 3603

Skopal, A. 1992, IBVS, 3780

Skopal, A. 1994, A\&A, 286, 453

Skopal, A. 1996, Ap\&SS, 238, 285

Skopal, A. 1997, in Physical Processes in Symbiotic Binaries, ed. J. Mikolajewska (Warsaw: Copernicus Foundation for Polish Astronomy), 99

Skopal, A. 1998, A\&A, 338, 599

Skopal, A. 2000, Contrib. Astron. Obs. Skalnate Pleso, 30, 21

Skopal, A. 2001a, A\&A, 366, 157

Skopal, A. 2001b, Contrib. Astron. Obs. Skalnate Pleso, 31, 119

Skopal, A. 2003a, A\&A, 401, L17 
Skopal, A. 2003b, New Astron., 8, 481

Skopal, A., 2003c, Recent Res. Devel. Astronomy \& Astrophys., 1, 111 [arXiv: astro-ph/0308462]

Skopal, A. 2005, in The Astrophysics of Cataclysmic Variables and Related Objects, ed. J. M. Hameury, \& J. P. Lasota, ASP Conf. Ser., 330, 463

Skopal, A., Bode, M. F., Lloyd, H. M., \& Tamura, S. 1996, A\&A, 308, L9

Skopal, A., Vittone, A., Errico, L., et al. 1997, MNRAS, 292, 703

Skopal, A., Bode, M. F., Lloyd, H. M., \& Drechsel, H. 1998, A\&A, 331, 179

Skopal, A., Djurašević, G., Jones, A., et al. 2000, MNRAS, 311, 225

Skopal, A., Teodorani, M., Errico, L., et al. 2001a, A\&A, 367, 199

Skopal, A., Kohoutek, L., Jones, A., \& Drechsel, H. 2001b, IBVS, 5195

Skopal, A., Bode, M. F., Crocker, M. M., et al. 2002a, MNRAS, 335, 1109

Skopal, A., Vaňko, M., Pribulla, T., et al. 2002b, Contrib. Astron. Obs. Skalnaté Pleso, 32, 62 [arXvi : astro-ph/0202249]

Skopal, A., Pribulla, T., Vaňko, M., et al. 2004, Contrib. Astron. Obs. Skalnaté Pleso, 34, 45 [arXiv: astro-ph/0402141]
Smith, V. V., Cunha, K., Jorissen, A., \& Boffin, H. M. J. 1996, A\&A, 315,179

Smith, V. V., Cunha, K., Jorissen, A., \& Boffin, H. M. J. 1997, A\&A, 324, 97

Stanishev, V., Zamanov, R., Tomov, N., \& Marziani, P. 2004, A\&A, 415,609

Tamura, S. 1983, PASJ, 35, 317

Taranova, O. G. 2000, Pisma v AZh, 26, 472

Taranova, O. G., \& Yudin, B. F. 1986, AZh, 63, 151

Tomov, N., \& Tomova, M. 1998, IBVS, 4574

Tomov, N., \& Tomova, M. 2001, Ap\&SS, 278, 311

Viotti, R., Badiali, M., Cardini, D., Emanuele, A., \& Iijima, T. 1997, in ESA-SP-402, Hipparcos, Venice 1997, ed. B. Batrick (Nordwijk: ESA), 405

Vogel, M. 1991, A\&A, 249, 173

Vogel, M., \& Nussbaumer, H. 1994, A\&A, 284, 145

Warner, B. 1995, Cataclysmic Variable Stars (New York: CUP), 43

Wheatley, P. J., Mukai, K., \& de Martino, D. 2003, MNRAS, 346, 855

Young, J. S., Baldwin, J. E., Boysen, R. C., et al. 2000, Proc. SPIE-4006, Interferometry in Optical Astronomy, (Munich: SPIE), 472 